NOTE TO USERS

This reproduction is the best copy available.

UMI 



\title{
INTEGRATED DETECTION OF ACTIVE WORMS USING MULTI-SENSOR DATA FUSION AND COLLABORATIVE NETWORK DEFENSE
}

\author{
By \\ FRANK ONYEKACHI AKUJOBI, B.SC., M.A.SC.
}

A thesis submitted to the Faculty of Graduate Studies and Research in partial fulfillment of the requirements

for the degree of

Doctor of Philosophy

(Electrical and Computer Engineering)

Ottawa-Carleton Institute for Electrical and Computer Engineering (OCIECE)

Department of Systems and Computer Engineering

Carleton University

Ottawa, Ontario, Canada, K1S 5B6

2009

(C) 2009 


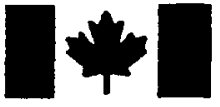

Library and Archives

Canada

Published Heritage

Branch

395 Wellington Street

Ottawa ON K1A ON4

Canada
Bibliotheque et

Archives Canada

Direction du

Patrimoine de l'édition

395 , rue Wellington

Ottawa ON K1A ON4

Canada
Your file Votre référence

ISBN: 978-0-494-63867-5

Our file Notre refférence

ISBN: 978-0-494-63867-5
NOTICE:

The author has granted a nonexclusive license allowing Library and Archives Canada to reproduce, publish, archive, preserve, conserve, communicate to the public by telecommunication or on the Internet, loan, distribute and sell theses worldwide, for commercial or noncommercial purposes, in microform, paper, electronic and/or any other formats.

The author retains copyright ownership and moral rights in this thesis. Neither the thesis nor substantial extracts from it may be printed or otherwise reproduced without the author's permission.
AVIS:

L'auteur a accordé une licence non exclusive permettant à la Bibliothèque et Archives Canada de reproduire, publier, archiver, sauvegarder, conserver, transmettre au public par télécommunication ou par l'Internet, prêter, distribuer et vendre des thèses partout dans le monde, à des fins commerciales ou autres, sur support microforme, papier, électronique et/ou autres formats.

L'auteur conserve la propriété du droit d'auteur et des droits moraux qui protège cette thèse. $\mathrm{Ni}$ la thèse ni des extraits substantiels de celle-ci ne doivent être imprimés ou autrement reproduits sans son autorisation.
In compliance with the Canadian Privacy Act some supporting forms may have been removed from this thesis.

While these forms may be included in the document page count, their removal does not represent any loss of content from the thesis.
Conformément à la loi canadienne sur la protection de la vie privée, quelques formulaires secondaires ont été enlevés de cette thèse.

Bien que ces formulaires aient inclus dans la pagination, il n'y aura aucun contenu manquant. 


\section{Abstract}

Fast spreading malicious worms have been known to cause severe havoc on networks they attack. Developing adequate detection and defense mechanisms against such worms with minimal false detection rates and optimized accuracy is therefore of keen interest. Modeling the behavior of fast worm detection and defense techniques to better understand and measure their effectiveness is crucial to developing effective defenses. Detection of slow scanning worms is also known to be particularly difficult due to the stealthy nature of slow worm propagation and their ability to blend with normal traffic patterns. The speed of fast scanning worms and the stealthiness of slow scanning worms pose unique challenges to malicious worm detection and defense.

This thesis develops a novel distributed detection and collaborative containment technique which we refer to as the EDANC (Endpoint Detection And Network Containment) technique for defending against fast spreading worms. The EDANC detection and correlation engine is based on the Generalized Evidence Processing (GEP) theory, a decision level multi-sensor data fusion technique. With GEP theory, evidence collected by distributed detectors determines the probability associated with a detection decision under a hypothesis. Several pieces of evidence are combined to arrive at an improved fused decision by minimizing a cummulative decision risk function. The EDANC scheme also employs automated collaborative network-centric containment for worm defense. Further, this thesis develops the Analytical Active Worm Containment (AAWC) model, a novel non-deterministic discrete-time model used to model vulnerable host population protected as a result of the EDANC collaborative defense mechanism in a large scale network. Analysing the AAWC model alongside a known discrete-time worm propagation model, this thesis demonstrates quantitatively the effectiveness of the EDANC technique in defending against large scale fast spreading scanning worm attacks.

Typically, techniques optimized for detecting fast scanning worms fail to detect slow scanning worms, and vice versa. While malicious traffic flows of varying scanning rates can occur concurrently in computer networks, the difficulty in detecting slow worms is exacerbated by interference from other traffic flows scanning at faster rates. This thesis formulates the problem of slow worm detection to include detection of faster scanning malicious traffic and filtering of traffic profiles associated with detected fast worms to isolate the malicious slow worms. This insight led to the development of a novel GEP theory-based integrated detection technique for detecting both fast and slow scanning malicious worm activity even when they occur concurrently in a target computer network. 


\section{Dedication}

This work is dedicated to my family for their persistent support and encouragement throughout my doctoral program. I would like to first dedicate this work to my lovely wife Valerie Tamunodein Akujobi who has been very understanding, supportive and caring during the course of this work. I also wish to dedicate this work to my great sons Kelechi Frank Akujobi and Onyekachi Bryan Akujobi. They have been such wonderful blessings to our family.

For being there right from the beginning and for their unconditional love and encouragement, I also dedicate this work to my parents Sylvester Omeni Akujobi and Rose Nene Akujobi, as well as my brothers John Bosco Okechukwu Akujobi, Chinasa Emmanuel Akujobi, and Uche Christopher Akujobi. 


\section{Acknowledgments}

I would like to acknowledge the support of my supervisors Dr. Ioannis Lambadaris and Dr. Evangelos Kranakis. Their careful guidance and sound advice were invaluable in the successful completion of my doctoral research work. 


\section{Contents}

$\begin{array}{ll}\text { List of Figures } & \text { vii }\end{array}$

List of Tables $\quad$ ix

List of Acronyms $\quad$ x

List of Notations $\quad$ xii

1 Introduction $\quad 1$

1.1 Malicious intrusions and worms .................... 3

1.2 Introduction to malicious worm detection and defense techniques . . . . . 5

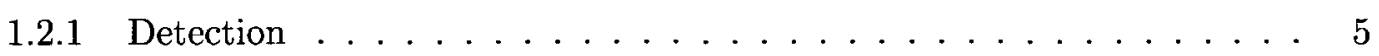

1.2 .2 Worm Defense ...................... 8

2 Thesis Approach and Contributions $\quad 11$

2.1 Detection of Fast Scanning Intrusions . . . . . . . . . . . 12

2.2 Modeling Collaborative Containment of Fast Scanning Intrusions . . . . . . . 13

2.3 Integrated Detection using Multi-sensor Data Fusion . . . . . . . . . . . 14 
3.1 Worm Propagation - Techniques and Models . . . . . . . . . . . 18

3.2 Anomaly-based Detection for Fast Scanning Worms . . . . . . . . . . 20

3.3 Worm Defense Techniques and Collaborative Containment . . . . . . . . . 22

3.4 Slow Worm Detection . . . . . . . . . . . . . . . 24

3.5 Integrated Detection Techniques $\ldots \ldots \ldots \ldots$

3.6 Multi-sensor Data Fusion - Introduction . . . . . . . . . . . . . 28

$\begin{array}{lll}4 & \text { Background } & 31\end{array}$

4.1 Decision Level Multi-sensor Data Fusion . . . . . . . . . . . . . 31

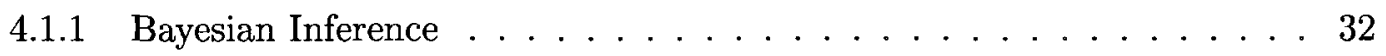

4.1.2 Dempster-Shafer Evidential Theory . . . . . . . . . . . . 34

4.1.3 Generalized Evidence Processing Theory . . . . . . . . . . . . . 35

5 EDANC Detection Technique for Fast Scanning Worms 43

5.1 Introduction . . . . . . . . . . . . . . . 43

5.2 The EDANC Scheme ......................... 46

5.2 .1 Detection Technique ..................... 47

5.2 .2 Correlation Technique .................. 48

5.2 .3 Containment Technique .................... 53

5.3 Detection Interval Analysis $\ldots \ldots \ldots \ldots \ldots$

5.3 .1 Total inter-infection interval, $t_{V, i} \ldots \ldots \ldots . \ldots . \ldots$ 
5.3 .2 Total time to infect, $t_{\text {infect }, i} \ldots \ldots \ldots \ldots \ldots$

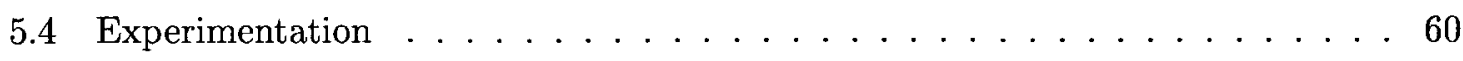

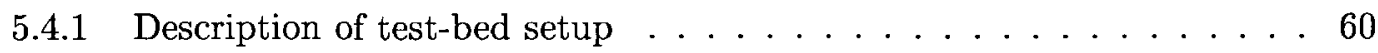

5.4.2 Experiment 1: EDANC detection interval ........... 62

5.4.3 Experiment 2: Effect of varying probability of detection, $p_{d}$ on EDANC

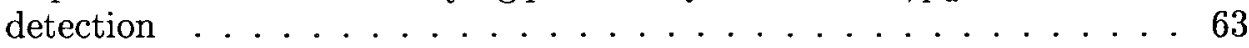

5.4.4 Experiment 3: Effect of varying probability of false detection, $p_{f}$ on EDANC detection .................... 64

5.4.5 Experiment 4: Effect of varying $\gamma=\frac{C_{10}}{C_{01}}$ on EDANC detection . . . 66

5.4.6 Experiment 5: Comparison: Accuracy of EDANC GEP-based detection versus single detector detection ............ 67

5.4.7 Experiment 6: Multiple concurrent worm attacks ........ 68

5.5 Chapter Summary . . . . . . . . . . . . . . 70

6 Modeling EDANC Collaborative Defense $\quad 73$

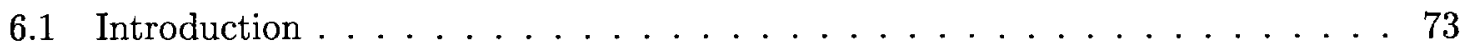

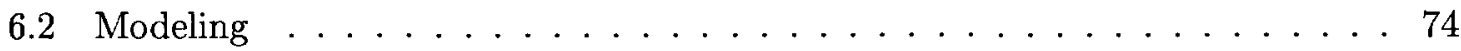

6.2.1 EDANC Protection Capability .................. 78

6.2 .2 EDANC with Immunization . . . . . . . . . . . . 81

6.3 Chapter Summary . . . . . . . . . . . . . . . 82

7 Integrated Detection using EDANC $\quad 85$

7.1 Introduction . . . . . . . . . . . . . . 85

7.2 Integrated Detection Approach $\ldots \ldots \ldots \ldots$ 


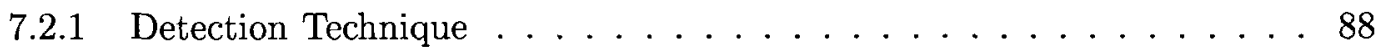

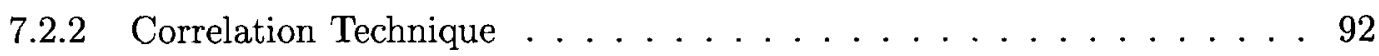

7.3 Experimentation and Analysis . . . . . . . . . . . . 97

7.3 .1 Description of test-bed setup ................... 97

7.3.2 Experiment 1: Integrated detection of fast and slow worms $\ldots \ldots 100$

7.3.3 Experiment 2: Effect of varying $\gamma_{f}$ and $\gamma_{s} \ldots \ldots \ldots 10 \ldots \ldots$

7.3.4 Experiment 3: Effect of varying $p_{f}$ on slow worm detection with background fast scanning benign traffic .............. 102

7.4 Chapter Summary . . . . . . . . . . . . . . 105

8 Conclusion and Future Directions $\quad 107$

8.1 Summary of Problems . . . . . . . . . . . . . . 107

8.2 Summary of Contributions . . . . . . . . . . . . 108

8.3 Summary of Thesis Content . . . . . . . . . . . . . 110

8.4 Future Directions . . . . . . . . . . . . . . . . . . 114

$\begin{array}{ll}\text { References } & 117\end{array}$ 


\section{List of Figures}

1.1 Taxonomy of worm detection techniques $\ldots \ldots \ldots \ldots$

1.2 Taxonomy of worm defense techniques $\ldots \ldots \ldots \ldots \ldots$

4.1 Transformation from $N$ local detector observations to a fused decision. $N=5 \ldots \ldots$. . . 36

4.2 Case 1: The indecision region lies between the two definite decision regions. . . . . . . . 39

4.3 Case $2:$ The indecision region is completely eliminated. . . . . . . . . . . 40

4.4 Case 3: The definite decision regions lies between two indecision regions. . . . . . . . . . . 40

5.1 Typical worm attack on multiple networks $\ldots \ldots \ldots \ldots$

5.2 Implementation of host detection of malicious intrusion on the $m^{\text {th }}$ DE. . . . . . . 50

5.3 Implementation of host detection of benign intrusion on the $m^{t h}$ DE. . . . . . . . 51

5.4 Distributed collaborative containment strategy $\ldots \ldots \ldots \ldots \ldots$

5.5 Average detection interval using (5.5), (5.6) and (5.7). $W=254, v_{i}=20 \ldots \ldots \ldots \ldots$

5.6 Test-bed used for experimentation $\ldots \ldots \ldots \ldots \ldots \ldots$

5.7 Experiment 1: Average detection interval. $W=254, \gamma=2.0, p_{d}=0.99, p_{f}=0.01 . \ldots \ldots 3$ 
5.8 Experiment 2: Impact of varying, $p_{d}$ on EDANC detection. $W=254, p_{f}=0.01$. Worm scanning rate $=20 \mathrm{~h} / \mathrm{s} . \gamma=2.0 . \ldots . \ldots \ldots \ldots \ldots$. . . . . . . . . . . . . . 64

5.9 Experiment 3: Impact of varying, $p_{f}$ on EDANC detection. $W=254$. Worm scanning rate $=$ $20 \mathrm{~h} / \mathrm{s}, \gamma=2.0, p_{d}=0.99$. . . . . . . . . . . . . . . . . . . . . . 65

5.10 Experiment 4: Impact of $\gamma=\frac{C_{10}}{C_{01}}$ on EDANC detection. $W=254, p_{d}=0.99, p_{f}=0.01 . . . .66$

5.11 Experiment 5: ROC curves for EDANC GEP detection versus single host detection. $W=254$. $\gamma=2.0$. . . . . . . . . . . . . . . . . . . . . . . . . 69

5.12 Experiment 6: Combined detection probability, $P_{D_{i}}, W=254, \gamma=2.0, p_{d}=0.9, p_{f}=0.01 . \quad . \quad . \quad 69$

6.1 Typical large-scale hierarchical network topology. . . . . . . . . . . . . . . . 75

6.2 Hierarchical network topology used in AAWC modeling. $\delta=1 . . . . . . . . . . . .76$

6.3 Effect of $r$ on protected population $M(j) . W=254, m=128 \ldots \ldots \ldots$

6.4 AAWC vs. AAWP. $z=30, y=3, s=3, t=6, p=0, r=30, \delta=1 \ldots \ldots$. . . . . . . . 81

6.5 Effect of network containment and immunization on infected population. $z=30, y=3, s=3, L=$

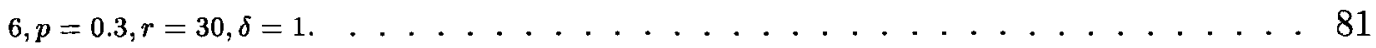

7.1 Typical worm attack on multiple networks . . . . . . . . . . . . . . . . . . . 87

7.2 Series of epochs showing detection windows . . . . . . . . . . . . . . . . 89

7.3 Communication flow between Detector Endpoints and Gateway Router . . . . . . . . . . 93

7.4 Block diagram of integrated detection of fast and slow worms. . . . . . . . . . . . . . 95

7.5 Test-bed used for experimentation . . . . . . . . . . . . . . . . . 98

7.6 Experiment 1: Integrated detection of fast and slow worms. $W=254, \gamma_{f}=2, \gamma_{s}=0.1, p_{f}^{s}=p_{f}^{f}=$ $0.01, p_{d}^{s}=p_{d}^{f}=0.99 . \ldots \ldots \ldots$. . . . . . . . . . . . . . . . . 101 
7.7 Experiment 2: Effect of varying $\gamma_{f}=\frac{C_{10}^{f}}{C_{01}^{f}}$ on fast worm detection in FWCE. $W=254, \gamma_{s}=0.1$. Worm scanning rate $=20 \mathrm{~h} / \mathrm{s}, p_{f}^{s}=p_{f}^{f}=0.01, p_{d}^{s}=p_{d}^{f}=0.99 . \ldots . . . . . . . . .103$

7.8 Experiment 2: Effect of varying $\gamma_{s}=\frac{C_{10}^{s}}{C_{01}^{s}}$ on slow worm detection in SWCE. $W=254, \gamma_{f}=2$. Worm scanning rate $=10 \mathrm{~h} / \mathrm{m}, p_{f}^{s}=p_{f}^{f}=0.01, p_{d}^{s}=p_{d}^{f}=0.99 . \ldots . . . . . . . . .103$

7.9 Experiment 3: Impact of varying $p_{f}^{s}$ on slow worm detection with background fast scanning benign traffic. $W=254$, Slow worm scanning rate $=10 \mathrm{~h} / \mathrm{m}, \gamma_{s}=0.1, \gamma_{f}=2.0, p_{d}^{s}=p_{d}^{f}=0.99, p_{f}^{f}=0.01 .104$ 


\section{List of Tables}

5.1 Relationship between $H_{1}, H_{0}$ and $p_{d}, p_{f}$ for individual detectors ...... 50

5.2 Parameters for GEP-based correlation algorithm . . . . . . . . . . 51

6.1 Parameters for network topology and AAWC model . . . . . . . . . 75

6.2 Parameters for AAWP Model . . . . . . . . . . . . . 79

7.1 Detection Parameters . . . . . . . . . . . . . 88

7.2 Parameters for Integrated GEP-based correlation algorithm . . . . . . . . . 92

7.3 Relationship between $H_{1}, H_{0}$ and $p_{d}^{f}, p_{f}^{f}$ for FWDA $\ldots \ldots \ldots 9$

7.4 Relationship between $H_{1}, H_{0}$ and $p_{d}^{s}, p_{f}^{s}$ for SWDA . . . . . . 99

7.5 Worm attack sources and scanning rates used in Experiment $1 \ldots$. . . . 100 


\section{List of Acronyms}

EDANC Endpoint Detection and Network Containment

GEP Generalized Evidence Processing

AAWC Analytical Active Worm Containment

DDoS Distributed Denial of Service

NATO North Atlantic Treaty Organization

DMZ Demilitarized Zone

ISP Internet Service Provider

SI Model Susceptible-Infected Model

AAWP Analytical Active Worm Propagation

RCS Random Constant Spread

AIDS Anomaly based Intrusion Detection System

IDS Intrusion Detection System

SWORD Self-propagating Worm Observation and Rapid Detection

DE Detector Endpoint 
GR Gateway Router

DA Detector Agent

FWDA Fast Worm Detector Agent

SWDA Slow Worm Detector Agent

FWCE Fast Worm Correlation Engine

FWCA Fast Worm Correlation Algorithm

SWCE Slow Worm Correlation Engine

SWCA Slow Worm Correlation Algorithm

ROC Receiver Operating Characteristics 


\section{List of Notations}

\begin{tabular}{|l|l|}
\hline Notations & Explanation of $\boldsymbol{E D A N C}$ Notations \\
\hline $\mathrm{h} / \mathrm{s}$ & Hosts per second \\
\hline $\mathrm{h} / \mathrm{m}$ & Hosts per minute \\
\hline$r$ & Worm scanning rate \\
\hline$P_{D i}$ & Combined probability of positive detection for traffic profile $i$ \\
\hline$p_{d q}$ & Detection probability for the $q^{\text {th }}$ individual detector \\
\hline$p_{f q}$ & False detection probability for the $q^{\text {th }}$ individual detector \\
\hline$d_{q}^{i}$ & Individual decision by the $q^{\text {th }}$ DE on intrusion attempts due to profile $i$. \\
\hline$\underline{d}^{i}$ & Vector of local host decisions concerning profile $i$ \\
\hline$t_{f}$ & Duration of fast worm detection window \\
\hline $\mathbf{D}$ & Optimal fused decision at the fusion center (gateway router) \\
\hline$\Lambda\left(\underline{d}^{i}\right)$ & GEP likelihood ratio for optimal fused decision \\
\hline$\gamma$ & GEP likelihood ratio threshold, also equivalent to $C_{10}$ \\
\hline$C_{a b}$ & Cost associated with a detector decision $a$ when the true hypothesis is $H_{b}$ \\
\hline$m_{i}$ & Total number of detectors with observations of profile $i$ \\
\hline$u_{i}$ & Total number of detectors with observations of profile $i$ and that favor $H_{1}$ \\
\hline$v_{i}$ & Number of detectors which favor $H_{1}$ required to minimally satisfy $\Lambda\left(\underline{d^{i}}\right) \geq \gamma$ \\
\hline$R_{i}$ & Computed probing rate for profile $i$ on gateway router \\
\hline$t_{D, i}$ & Detection interval for worm with profile $i$ \\
\hline$t_{V, i}$ & Total inter-infection interval for worm with profile $i$ \\
\hline
\end{tabular}




\section{List of Notations (continued)}

\begin{tabular}{|c|c|}
\hline Notation & Explanation of $E D A N C$ Notations \\
\hline$t_{\text {infect }, i}$ & Total time it takes worm with profile $i$ to infect a vulnerable host \\
\hline$L$ & Number of hierarchical levels in network \\
\hline$y$ & Number of nodes that connect to an upstream node \\
\hline$W$ & Number of hosts in each network segment or cell targeted by worm attack \\
\hline$s$ & Number of W-sized cells that exist on each GR \\
\hline$t_{r}$ & Time intrusion traffic is released into the network \\
\hline$t_{j}$ & Time of containment at level $j$ in the hierarchical network \\
\hline$\delta$ & Time interval for notification between routers \\
\hline$M\left(t_{j}\right)$ & Total number of contained hosts after a time interval of $t_{j}$ \\
\hline$H$ & Total number of vulnerable machines \\
\hline$p$ & Patching rate - rate an infected or vulnerable machine becomes invulnerable \\
\hline$z$ & Size of hitlist - \# of infected machines at beginning of worm spread \\
\hline$d$ & Death rate - rate an infection is detected and eliminated without patching \\
\hline$n_{i}$ & Number of infected machines at time tick $t_{i}$ \\
\hline$h_{i}$ & Number of vulnerable machines (including infected ones) at time tick $t_{i}$ \\
\hline
\end{tabular}




\section{List of Notations (continued)}

\begin{tabular}{|l|l|}
\hline Notation & Explanation of Integrated Detection Notations \\
\hline$S W_{j}$ & $j^{\text {th }}$ slow worm detection window \\
\hline$F W_{k j}$ & $k^{\text {th }}$ fast worm detection window within $S W_{j}$ \\
\hline$t_{s}$ & Duration of slow worm detection window \\
\hline$t_{f}$ & Duration of fast worm detection window \\
\hline$Z_{j}$ & Set of profiles captured by the SWDA during $S W_{j}$ \\
\hline$X_{k j}$ & Worm profiles detected during the $W_{k j}$ window \\
\hline$Y_{j}$ & Set of profiles forwarded to the slow worm correlation engine (SWCE) \\
\hline$P_{D i}^{f}$ & Combined probability of detection for traffic profile $i$ on FWCE \\
\hline$P_{D j}^{s}$ & Combined probability of detection for traffic profile $j$ on SWCE \\
\hline$p_{d q}^{f}$ & Detection probability for the FWDA on the $q^{\text {th }}$ DE \\
\hline$p_{f q}^{s}$ & False detection probability for the FWDA the $q^{\text {th }}$ DE \\
\hline$p_{d q}^{f}$ & Detection probability for the SWDA on the $q^{t h}$ DE \\
\hline$p_{f q}^{s}$ & False detection probability for the SWDA the $q^{\text {th }}$ DE \\
\hline$d_{f q}^{i}$ & Individual decision by FWDA on the $q^{\text {th }}$ DE on intrusion attempts due to profile $i$. \\
\hline$d_{s q}^{j}$ & Individual decision by SWDA on the $q^{\text {th }}$ DE on intrusion attempts due to profile $j$. \\
\hline $\mathbf{D}^{\mathbf{f}}$ & Fused decision on FWCE. \\
\hline $\mathbf{D}^{\mathbf{s}}$ & Fused decision on SWCE. \\
\hline$\Lambda\left(d_{f}^{i}\right)$ & GEP likelihood ratio for optimal fused decision on FWCE about profile $i$ \\
\hline
\end{tabular}




\section{List of Notations (continued)}

\begin{tabular}{|c|l|}
\hline Notation & Explanation of Integrated Detection Notations \\
\hline$C_{a b}^{s}$ & Cost associated with a detector decision $a$ when the true hypothesis is $H_{b}$ on SWCE \\
\hline$\gamma_{f}$ & GEP likelihood ratio threshold on the FWCE, also equivalent to $\frac{C_{1}^{f}}{C_{0}^{f}}$ on the FWCE \\
\hline$\gamma_{s}$ & GEP likelihood ratio threshold on the SWCE, equivalent to $\frac{C_{s}^{s}}{C_{01}^{s}}$ on the SWCE \\
\hline$m_{i}^{f}$ & Total number of FWDAs with observations of profile $i$ \\
\hline$u_{i}^{f}$ & Total number of FWDAs with observations of profile $i$ and that favor $H_{1}$ \\
\hline$v_{i}^{f}$ & Number of FWDAs which favor $H_{1}$ required to minimally satisfy $\Lambda\left(\underline{d_{f}^{i}}\right) \geq \gamma$ \\
\hline$m_{j}^{s}$ & Total number of SWDAs with observations of profile $j$ \\
\hline$u_{j}^{s}$ & Total number of SWDAs with observations of profile $j$ and that favor $H_{1}$ \\
\hline$v_{j}^{s}$ & Number of SWDAs which favor $H_{1}$ required to minimally satisfy $\Lambda\left(\underline{d_{s}^{j}}\right) \geq \gamma$ \\
\hline$\Lambda\left(\underline{d_{s}^{j}}\right)$ & GEP likelihood ratio for optimal fused decision on SWCE about profile $j$ \\
\hline$C_{a b}^{f}$ & Cost associated with a detector decision $a$ when the true hypothesis is $H_{b}$ on FWCE \\
\hline
\end{tabular}




\section{Chapter 1}

\section{Introduction}

Self-propagating programs also known as worms have become an interesting network security research focus. Their complex propagation techniques which enable them replicate themselves across networks and from machine to machine as well as and the wide-spread nature of malicious worm attacks have made the study of worms very crucial. The consequence of such attacks are also known to be severe. Several recent publications attempt to provide better understanding of the nature and behavior of malicious worms. Modeling worm propagation mechanisms and assessing the risk and implication of a malicious worm epidemic to network infrastructures and computer network services in general has also attracted huge attention.

Similarly, significant effort has been invested in developing detection and defense mechanisms against malicious worm attacks. Several techniques have been proposed to achieve some form of worm defense *. While some proposals for malicious worm defense have been adopted by the industry and incorporated into vendor products, others seem to be more theoretical than practically useful in today's computer network environments due to high false detection

* We consider worm defense to include all actions required to defend against a worm attack. This includes worm detection and worm containment actions. 
rates. However, the threat of large scale worm attacks remain quite formidable and real as is evident in recent successful attacks and analysis [46] [57] [83] [58] [20] [41].

While providing further insight into the threat posed by malicious worms on computer networks like the Internet and large enterprise networks, this thesis attempts to achieve the following:

1. Development of a technique for fast detection and containment of fast scanning malicious worms in computer networks with low false detection rates and improved accuracy irrespective of their signature or their scanning strategies. Detection of such worms with minimal false detection rates and within short intervals still remains a problem today and development of a practical and deployable detection and containment solution remains an open research issue.

2. Development of a technique for detection of both slow scanning worms and fast scanning malicious worms in computer networks with low false detection rates and improved accuracy even when they occur concurrently in a target computer network. We point out that there are two major shortfalls of previously proposed approaches to detection of slow scanning and fast scanning worms. First, a common characteristic of previously proposed techniques is the use of connection counts and traffic rates as the basis for anomalous detection. This approach inherently carries a high rate of false positives and false negatives because slow worms are capable of propagating at rates similar or less than normal traffic rates and therefore can camouflage as normal traffic. Also, anomalous connection counts and traffic rates (whether high or low) do not necessarily confirm existence of malicious activity. Second, though previously proposed techniques demonstrate capability to detect fast scanning and slow scanning intrusions under certain circumstances, they do not address detection of both fast scanning and slow scanning malicious intrusions when they occur concurrently in the same target computer network. With advancements in worm propagation techniques and consid- 
ering the wide range of worm exploits that have been seen on the Internet and in computer networks, a detection approach that takes into account the real possibility that slow scanning malicious worms can occur concurrently with faster propagating malicious worms in computer networks is required.

\subsection{Malicious intrusions and worms}

The inherent nature of worms which enable them spread autonomously across several computer machines also makes them a vehicle for spreading malicious computer programs. Security vulnerabilities exist in computer machines primarily due to programming flaws in computer software code [71] [33]. Proactive prevention of software security vulnerabilities can only be achieved by good software development practices [71], but this is far from being achievable today. Malicious programs such as worms exploit security vulnerabilities in computer software and compromise the target computer machine. The effect of such malicious programs on compromised vulnerable machines vary widely depending on the design intent of the malicious program. While some malicious programs inflict direct damage on infected systems, others leave backdoors for future attacks and malicious remote control of the systems. The resultant impact is a loss or degradation of service on compromised systems as well as significant financial loss to organizations that rely on networked systems. The three largest worms of 2003, Sobig, Nachi, and Blaster affected systems globally and caused close to $\$ 5$ billion in damages [20]. From February 2004 to May 2004, several variants of MyDoom, Netsky, Bagel and Sasser worms caused over $\$ 11$ billion in damages worldwide [20]. Worms that leave backdoors on systems thus exposing the systems to future malicious intrusions are also used by hackers to remotely control compromised systems. Such systems are called zombies and the piece of software that allows a system to be remotely controlled without the system owner's knowledge is called a bot. A group of zombies controlled by a hacker is refered to as a botnet. Some botnets have been known to comprise tens of thou- 
sands of machines and have been used to launch distributed denial of service (DDoS) attacks. During a typical DDoS attack, zombie machines are instructed to inundate a target system with thousands of packets of data in an attempt to overwhelm the system and cause it to crash. In 2000, e-commerce sites belonging to eBay, Yahoo and Amazon were the subject of DDoS attacks resulting in a cummulative loss of $\$ 1.2$ billion, and in May of 2001 , the whitehouse.gov site fell under a barrage of denial of service assaults [41]. An estimated $\$ 500$ million was lost by Microsoft in 2001 due to two days of disrupted network connectivity as a result of a DDoS attack [40]. Data such as these has forced the quest for effective defenses against worm-related attacks to preserve productivity and business continuity for today's organizations. DDoS attacks have also been recently used for political gains. According to [83], Estonia's national IT infrastructure was recently subjected to a series of DDoS attacks following a political fall out with Russia. Subsequently, NATO (North Atlantic Treaty Organization) finalized its first policy covering cyber attacks on member states' critical national infrastructure. In North America, government departments too have indicated strong interests in funding and developing effective detection and defense strategies against such attacks as a matter of national security [78]. Home users with Internet connected systems are not spared either. Most zombie machines belong to this group of users who typically are not computer security savvy and are therefore more vulnerable to worm-based malicious attacks. Infections on home-based machines interfere with home-based businesses and affect the quality of life of recreational home computer users.

The security threat posed by malicious computer worms therefore cuts across virtually all sectors of business and human endeavour that rely on networked systems. The impact of a successful malicious worm attack can be devastating with severe implications for individuals, large businesses, and national governments.

Several recent literature attempt to provide better understanding of the nature and behavior of worms. Modeling worm propagation mechanisms and assessing the risk and implication of a worm epidemic on network infrastructure and computer network services in general 
has attracted huge attention. Similarly, significant effort has been invested in developing detection and defense mechanisms against worm attacks. While some proposed worm detection and defense techniques have been adopted by the industry and incorporated into vendor products, others seem to be more theoretical than practically useful in today's computer network environments. However, the threat of large scale worm attacks remain quite formidable and real in computer networks today.

\subsection{Introduction to malicious worm detection and defense tech- niques}

\subsubsection{Detection}

Techniques for detecting malicious intrusions can be divided into two broad groups: Signature detection techniques and anomaly detection techniques. Fig. 1.1 depicts a taxonomy for worm detection techniques. A malicious code signature is an invariant component of that malicious code that can be used to uniquely identify the malicious code. Signature detection techniques in general require a managed database of unique malicious code signatures and are only capable of detecting malicious packets with known signatures [76]. Using the point of detection as a basis for classification, signature detection techniques can be classified as either host-based or network-based detection techniques. Host-based signature detection techniques maintain the database of known malicious signatures on endpoints used for detection. Use of host-based anti-malware and anti-virus software fall within this class of detection. Network-based signature detection techniques such as network intrusion detection systems inspect network traffic at designated points in the network for presence of known malicious code in packet payloads. While signature detection techniques can be used to detect re-occurring malicious attacks that are known and for which signatures have been developed, zero-day attacks due to malicious traffic with unknown signatures cannot 


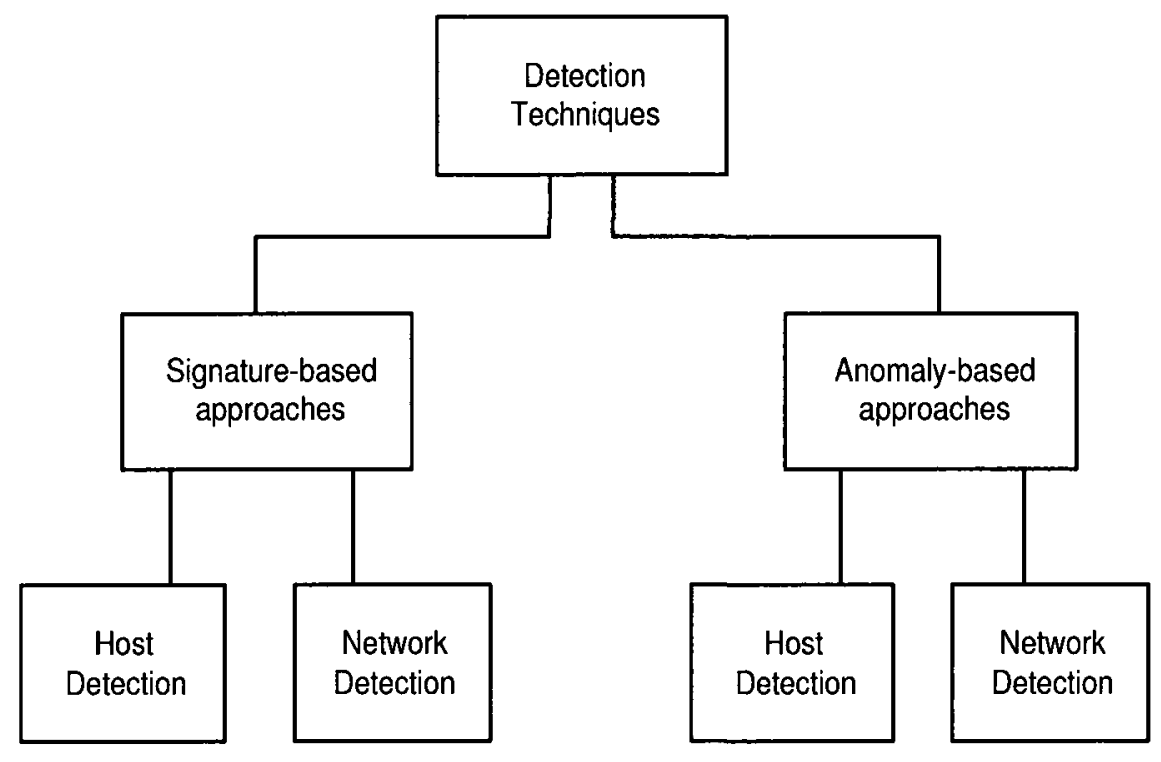

Figure 1.1: Taxonomy of worm detection techniques

be detected by such techniques. Similarly, intrusion defense and containment systems that depend on signature-based detection schemes are largely ineffective in defending against zero-day attacks. Also, there are well-known techniques for evading signature-based detection. Such techniques include encryption and tunneling of malicious traffic flows to hide the signature of the code [44] [32] [64]. Packet fragmentation can also be used to evade detection since some network-based signature detection schemes may not re-construct the complete datagram or may not correctly re-assemble out-of-order fragments [32]. Recently, the use of polymorphic mutations which change the byte sequence of different worm instances to evade signature based intrusion detection systems was also affirmed [25].

Anomaly detection techniques can also be classified as network-based or host-based depending on where the anomalous behavior is to be detected. Network-based anomaly detection techniques infer malicious activity in a network by detecting some form of anomalous network traffic patterns [10] [29] [45]. In [60], the number of connection attempts and failures was used for such network anomalous detection. ARP requests and name resolution requests or lack of them have also been used in [80] [79] as a basis for anomalous detection in the 
network. Network-based anomaly detection techniques are somewhat limited in their ability to accurately detect worm activity since there is no information about vulnerabilies expoited by worms at the network level [21]. The lack of such verifiable malicious activity results in non-trivial amounts of false positives and false negatives. Also, while network-based anomaly detection techniques can effectively detect fast spreading worms under favorable conditions, they cannot reliably detect worm propagation that does not cause anomalous traffic patterns [25] [66]. For instance, flash worms [66] do not exhibit anomalous number of connection failures and connection attempts since they have pre-determined lists of vulnerable targets. Such worms will therefore evade existing scanning worm detection techniques that depend solely on number of connection failures and connection attempts for detection.

Host-based anomaly detection techniques monitor specific endpoint and system-level characteristics and infer malicious activity when normal system behavior is altered. Recent research work [74] [21] [44] [9] and vendor implementations [68] have recorded success in using host-based anomaly intrusion detection techniques for effective detection of unauthorized intrusions. Host-based anomaly detection techniques are capable of leveraging large amounts of detailed context about applications and system behavior to effectively detect anomalous host behaviors [64]. Authors in [9] presented a detection model that successfully detects attacks on a host machine running Windows by checking for anomalous attempts to access the Windows registry. It has been suggested that since end hosts running vulnerable software are the targets of malicious code attacks, they ought to be the point of detection [21]. The host-based anomaly detection technique adopted in [21] shows that with properly instrumented detection software, host-based intrusion detection is effective and capable of eliminating false positives. 


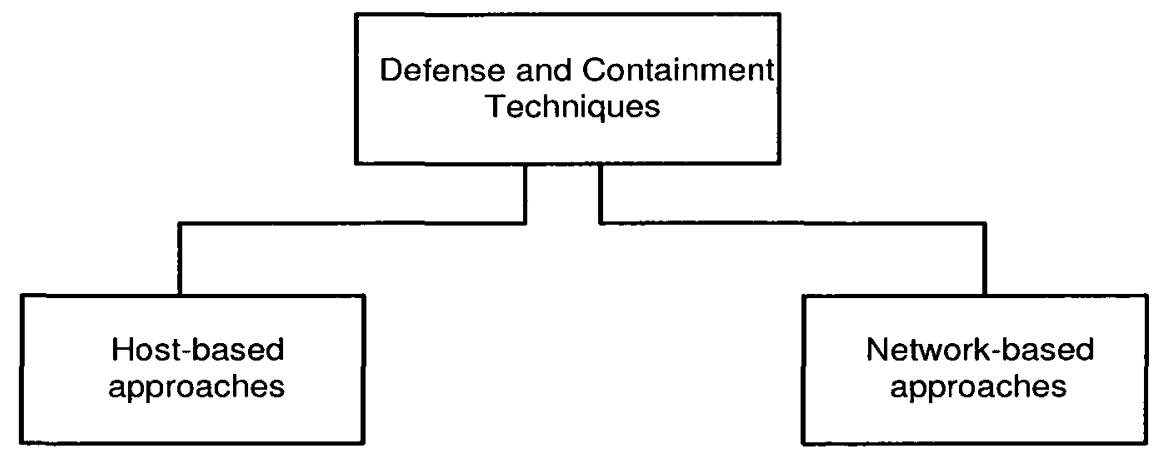

Figure 1.2: Taxonomy of worm defense techniques

\subsubsection{Worm Defense}

Similar to detection, worm defense and containment techniques can be classified as hostbased or network-based techniques (Fig. 1.2). Host-based defense techniques typically involve deployment of system patches [44] against detected malicious code or activation of host-based filters [21] to block malicious intrusion traffic. Automated host-based defenses can be successfully deployed in specific scenarios or network zones such as in server farms or in a demilitarized network zone (DMZ). However, host-based techniques are not very scalable and are unlikely to address worm containment in large enterprise networks. Large scale fast spreading worm attacks in particular that traverse several ISP and enterprise networks would be difficult to contain using host-based techniques. Also, in large networks, network administrators do not typically have any direct control over individual client machine configuration and therefore cannot depend on them for worm containment. Another problem with host-based defense techniques is that a successful compromise of a participating host could subvert the entire defense strategy [77] [56].

Network-based defense techniques typically implement filters on network devices such as firewalls, routers and intrusion prevention systems to block malicious intrusion traffic. In [50], content filtering based on signatures was compared to network address blocking techniques and it was shown that if the reaction time between detection and containment can 
be minimized, address blocking techniques can be more effective. Recent developments in router technology which enables deep packet inspection and content-based filtering [70] has been leveraged for network-based defenses. 


\section{Chapter 2}

\section{Thesis Approach and Contributions}

This thesis work investigates the following problems related to malicious worms and intrusions in computer networks and develops solutions to address them:

1. Fast detection and containment of fast scanning malicious worms in computer networks with low false detection rates and improved accuracy irrespective of their signature or their scanning strategies. Detection of such worms with minimal false detection rates and within short intervals still remains a problem today and development of a practical and deployable detection and containment solution remains an open research issue.

2. Detection of both slow scanning stealthy worms and fast scanning malicious worms in computer networks with low false detection rates and improved accuracy even when they occur concurrently in a target computer network. We point out that there are two major shortfalls of previously proposed approaches [1] [61] to detection of slow scanning and fast scanning worms.

$\dagger$ First, a common characteristic of previously proposed techniques is the use of 
connection counts and traffic rates as the basis for anomalous detection. This approach inherently carries a high rate of false positives and false negatives because worms are capable of propagating at rates similar or less than normal traffic rates and therefore can camouflage as normal traffic [27]. Also, anomalous connection counts and traffic rates (whether high or low) do not necessarily confirm existence of malicious activity.

† Second, though previously proposed techniques [1] [61] demonstrate capability to detect fast scanning and slow scanning intrusions under certain circumstances, they do not address detection of both fast scanning and slow scanning malicious intrusions when they occur concurrently in the same target computer network. This particularly exacerbates the difficulty in detecting slow worms due to interference from other traffic flows scanning at faster rates. With advancements in worm propagation techniques and considering the wide range of worm exploits that have been seen in the wild, a detection approach that takes into account the real possibility that slow scanning malicious worms can occur concurrently with faster propagating malicious worms in computer networks is required.

\subsection{Detection of Fast Scanning Intrusions}

The first contribution of this thesis to the state of the art is the novel use of the Generalized Evidence Processing (GEP) theory, an optimized decision level multi-sensor data fusion technique for detection of fast scanning malicious intrusions such as worms. The GEP theory ensures an improved fused decision is made by minimizing the cummulative risk associated with making that decision. Other popular decision level multi-sensor data fusion techniques, such as the Bayesian theory and the Dempster-Shafer theory have been used in previous works as theoretical foundations for intrusion detection techniques [82] [18] [12], but as far as our literature survey revealed, and to the best of our knowledge, we were 
the first in [4] to use the GEP theory as a theoretical foundation for intrusion detection of malicious traffic in computer networks. The GEP theory technique has also been known to have merits over both the Bayesian and Dempster-Shafer techniques [39]. Using the GEP theory, we developed a detection and correlation engine for our novel Endpoint Detection And Network Containment (EDANC) approach for distributed detection and collaborative defense against fast spreading worms.

\subsection{Modeling Collaborative Containment of Fast Scanning In- trusions}

The second contribution of this thesis is the development and analysis of the Analytical Active Worm Containment (AAWC) model. The AAWC model is a novel non-deterministic discrete-time model which we use to model vulnerable host population protected from random scanning fast spreading malicious worms as a result of the EDANC collaborative defense scheme in a large scale network. Using the AAWC model, we demonstrate quantitatively the collaborative containment capability of EDANC defense in a large scale network.

The proposed EDANC approach uses a network-based collaborative technique for fast containment of fast spreading worms. With the EDANC approach, after the detection and correlation engines identify an offending traffic flow, network filters are automatically generated and deployed on the first upstream router (i.e the gateway router) of the network segment or cell under attack. The gateway router of the target cell then sends notification signals to neighbor routers. Participating neighbor routers that receive the notification signal run a reactive blocking protocol which ensures execution of a similar containment action if the offending traffic is still active. The neighbor routers then notify their own neighbor routers thus propagating the containment information to achieve enterprise-wide

collaborative containment. We used the AAWC model to quantitatively demonstrate the 
effectiveness of the EDANC containment technique in protecting a vulnerable uninfected population from infection from a fast scanning malicious worm attack. We define containment of an infectious worm as the complete halting of further worm spread. Therefore, when a fast spreading worm is contained using the EDANC defense technique, significant portions of the vulnerable uninfected population are protected from infection.

\subsection{Integrated Detection using Multi-sensor Data Fusion}

The third contribution of this thesis to the state of the art is the proposal and development of a novel approach to integrated detection of both slow scanning stealthy worms and fast scanning malicious worms that is distinct from previously proposed approaches. First, our proposed detection approach uses an optimized correlation technique based on the GEP theory to combine malicious intrusion evidence provided by distributed host-based anomaly intrusion detectors. The use of the GEP theory, an optimized multi-sensor data fusion technique for combining the local decisions provided by the individual host detectors ensures that false detection rates are minimized and that detection accuracy is optimized. The fact that host anomaly detectors are also known to be capable of exhibiting low false detection rates [21] [68] adds to making our novel GEP-based detection technique even more viable. Second, our novel detection approach takes into consideration the real possibility that slow scanning malicious worms can occur concurrently with faster propagating malicious worms in computer networks. This particularly exacerbates the difficulty in detecting slow worms due to interference from other traffic flows scanning at faster rates. We introduced an improvement to our proposed EDANC detection and correlation engine to extend its capabilities to detection of slow worms. The improved EDANC detection and correlation engine uses a detection window-based self adapting profiler to filter detected fast scanning malicious traffic profiles hence isolating detected slow scanning malicious traffic profiles in the correlation engine. The resulting integrated detection technique was used to achieve 
detection of both fast scanning and slow scanning malicious worms even when they occur concurrently in a target computer network. In Chapter seven, we present the integrated detection technique and use experiments on a live test-bed to demonstrate its functionality.

A summary of the main contributions of this thesis work are:

$\dagger$ Development of the EDANC defense scheme for detection and containment of fast spreading malicious worms. The detection and correlation engine is based on the Generalized Evidence Processing (GEP) theory, a decision-level multi-sensor data fusion technique, while a network-based collaborative technique is used for containment.

$\dagger$ Experiments on a live test-bed to validate the functionality and performance of the EDANC detection technique.

$\dagger$ Development of the Analytical Active Worm Containment (AAWC) model, a discretetime model used to model vulnerable host population protected from random fast scanning worms as a result of EDANC collaborative containment technique in a large scale network.

† Use of the AAWC model to demonstrate quantitatively the collaborative containment capability of the EDANC scheme. Containment of an infectious worm is defined as the complete halting of further worm spread. Therefore, when a fast spreading worm is contained using the EDANC defense, significant portions of the vulnerable uninfected population are protected from infection.

$\dagger$ We propose an integrated approach to detecting both fast and slow scanning malicious worms by introducing improvements to our EDANC scheme.

$\dagger$ We achieve detection of slow worms by using a combination of evidence from hostbased anomaly detectors, a detection window-based adaptive profiler and a detection engine based on the Generalized Evidence Processing (GEP) theory. 
$\dagger$ Our approach takes into consideration the real possibility that faster propagating malicious intrusions can occur concurrently with slow worms in computer networks, and therefore interfere with slow worm detection.

$\dagger$ Experimentation on a live test-bed to demonstrate the integrated detection technique and present results.

This thesis work resulted in the following technical publications:

† A journal paper, "Towards Host-based Detection and Collaborative Network Containment of Fast Spreading Active Worms", submitted to the Journal of Network and Computer Applications, 2009 [6].

† A conference paper, "Detection of Slow Malicious Worms using Multi-sensor Data Fusion", published in the proceedings of the IEEE Symposium on Computational Intelligence for Security and Defense Applications (CISDA 2009), 2009 [4].

$\dagger$ A conference paper, "An Integrated Approach to Detection of Fast and Slow Scanning Worms", published in the proceedings of the ACM Symposium on Information, Computer and Communications Security (ASIACCS 2009), 2009 [5].

$\dagger$ A conference paper, "Modeling Host-based Detection and Active Worm Containment", published in the proceedings of the 11th Communications and Networking Simulation Symposium (CNS), 2008 [3].

† A conference paper, "Endpoint-driven Intrusion Detection and Containment of Fast Spreading Worms in Enterprise Networks", published in the proceedings of the IEEE Military Communications Conference (MILCOM), 2007 [2]. 


\section{Chapter 3}

\section{Related Work}

This thesis work is concerned with developing an optimized detection and defense technique against malicious worm activity in computer networks. This work also focusses on the use of multi-sensor data fusion techniques for detecting both fast scanning malicious worm intrusions and slow scanning stealthy worms even when they occur concurrently in computer networks. In this chapter, we first discuss previous works related to worm propagation techniques and modeling worm propagation behavior. Second, we discuss previous works related to anomaly-based detection of fast scanning worms in computer networks. We also review previous works related to worm defense techniques and collaborative containment of fast scanning worms. We then examine slow worm detection and techniques for integrated detection of both fast and slow malicious worms related to our work. Finally, this chapter discusses previous works related to multi-sensor data fusion techniques used for intrusion detection. 


\subsection{Worm Propagation - Techniques and Models}

Fast spreading self-propagating malicious programs also known as active worms [67] [19] have been an enduring security threat on the Internet and large enterprise networks. With advanced scanning and propagation techniques, active worms are known to have successfully spread across the Internet in a few seconds [67]. The Code Red and the Slammer worms uniformly scanned the entire IPv4 space [48] [23]. Blaster sequentially scanned the Internet choosing to sequentially scan from a local IP address with $40 \%$ probability [24]. Code Red II also used a local preference scan in its propagation, exhibiting a higher probability of scanning an IP address within the same Class B or Class A network as the attacker than a random address [49]. Researchers have also conceived of advanced scanning techniques for highly virulent worms. With hit-list scanning [67], an attacker first builds a list of IP addresses of vulnerable hosts into the worm code before release of the worm. This significantly shortens the propagation time and improves effectiveness of the worm. The flash worm is an example of such a worm. With topological scanning [67], the worm uses information gathered from victim machines to create new target hosts. The Morris worm used this technique. A worm that successfully exploits a vulnerable host that is part of a peer-to-peer network can use this technique to spread across the peer-to-peer network by gathering a list of peers from the exploited host.

Worm propagation has been likened to biological virus spreading and biological epidemiological models have been used to model worm propagation [26] [38]. The simple epidemic model (SI model) assumed that the vulnerable population size is constant and that no recovery or death of an infected host is possible. An infected system therefore will remain in an infected state perpetually. Kermack-McKendrick's SIR model [26] [38] improved the SI model by considering that some infected host either recover from the infection or die with time. However, this model did not consider human countermeasures or automatic defense mechanisms which can reduce infection rates and isolate both infected and vulnerable sys- 
tems. The two-factor model [85] modeled Internet worm propagation by considering human coutermeasures such as patching, physical removal of systems from the network and manual setup of router filters. This model did not account for automated defenses which do not rely on human intervention. More analytical worm propagation models which are not derived from epidemiology have also been developed. The Analytical Active Worm Propagation (AAWP) model [19] is a discrete time model that characterizes the propagation of random scanning worms. In [19] the AAWP model was described as more accurate and realistic compared to the epidemiological models for the following reasons:

1. In the AAWP model a host cannot infect other hosts before it is completely infected, but in the epidemiological models a host begins infecting other hosts even before it is completely infected. Therefore the observed propagation speed of the worm and number of infected hosts are different with the two models. The AAWP model uses a more accurate approach.

2. The epidemiological models do not consider the time it takes to infect a host. Depending on characteristics such as size of the worm and the vulnerability exploited by the worm, scanning worms take a varying amount of time to infect a vulnerable host. The AAWP model takes this time into consideration and [19] shows that the time to infect a host is an important factor in the spread of active worms.

3. The AAWP model also takes into consideration the realistic case that a vulnerable host can be scanned by multiple copies of a worm at the same time. The epidemiological models ignore this case.

Besides AAWP model, the Random Constant Spread (RCS) worm model, also not derived from epidemiology, was developed in [67] to model the behavior of the Code Red worm [49]. The accuracy of the model was demonstrated in modeling the Slammer worm outbreak [48]. The RCS model assumes that an infected host randomly scans the IP address space for vulnerable machines. The model captures the initial worm spread at an exponential rate 
and eventual slow down as the worm spread reaches saturation. The RCS model shows that to be effective, practical worm detection and containment system must detect and initate rapid containment of the worm spread as early as possible in the exponential growth phase. In [59] it was noted that traditional RCS worm model is fundamentally deterministic and identified conditions under which variability in the stochastic behavior of the RCS worms can be ignored. Authors in [59] then presented a hybrid deterministic/stochastic model for a RCS worm behavior.

The importance of considering the stochastic behavior of worms in modeling worm propagation and in developing worm defenses was further emphasized in [55]. Using the Time of Next Infection (TNI) model, it was shown in [55] that the variance in worm infection time is primarily due to variance in inter-infection times early in the worm's life. According to [55], consideration for stochastic variance in the worm infection time is particularly crucial for quantitative evaluation of worm detection and defense mechanisms since metrics such as number of infections or scans as well as and reaction time of worm defense systems are affected by this variance.

\subsection{Anomaly-based Detection for Fast Scanning Worms}

Anomaly-based intrusion detection has emerged as a mechanism for detecting malicious intrusions that are either previously unknown or are capable of bypassing traditional signaturebased detection schemes [25] [32] [44]. Anomaly-based Intrusion Detection Systems (AIDS) can be classified as network AIDS or host AIDS depending on where the anomalous behavior is detected. Network AIDS infer suspicious activity in a network by detecting anomalous network traffic patterns [79] [10] [60]. While this approach can effectively detect fast spreading worms under favorable conditions, it cannot reliably detect malicious intrusions that do not cause anomalous network traffic patterns [25] [21] [60]. Host-based AIDS which infer 
suspicious activity when detector endpoints experience an intrusion that attempts to alter a pre-defined standard state ${ }^{*}$ of the endpoint have been more successful at detecting malicious worm intrusions irrespective of scanning behavior of worms. Typically, such attempts are in the form of anomalous system calls [51] or unauthorized intrusions [44] which cause the host AIDS to trigger an alert. This aligns with the argument in [21] that since end hosts running vulnerable software are the targets of malicious code attacks, they ought to be the point of detection. Recent work [21] [2] and vendor implementations [68] have recorded success in using host AIDS for detecting unauthorized intrusions. Host AIDS are capable of leveraging large amounts of detailed context about applications and system behavior to effectively detect anomalous host behaviors [64]. The technique adopted in [21] shows that with properly instrumented detection software, host-based intrusion detection is effective and capable of minimizing false positives. Though host-based AIDS can successfully detect malicious intrusions on a host and therefore determine the attempted exploit, the potential false positive rates and detection accuracy associated with individual host-based AIDS software is still a concern. Hence, some form of intelligent correlation and analysis is required to reduce the dependence on a single host anomaly detector and therefore minimize false alarm rates and improve detection accuracy. This is especially crucial for detection of multiple concurrent malicious attacks on a computer network. In [28] [17], this concept of combining alerts from multiple detectors for improved performance was also emphasized. Authors in [28] proposed a likelihood ratio test (LRT) technique for combining different alarm reports and showed that with the LRT technique detection accuracy can be improved to some degree.

In this thesis, we use distributed anomaly-based host detectors for local detection of fast scanning malicious worms. Reports from the local detectors are combined in a fusion center running our novel correlation algorithm based on a decision level multi-sensor data fusion technique.

${ }^{*}$ Pre-defined standard states of endpoints are typically determined by established security policies and standards. 


\subsection{Worm Defense Techniques and Collaborative Containment}

The advanced scanning and propagation techniques of malicious worms in computer networks and the severity of the damage they inflict on vulnerable computer systems has motivated several efforts to understand worm propagation mechanisms as well as contrive techniques for actively defending against malicious worm attacks. Active defense mechanisms take the battle to the worm [53] by automatically eliminating, isolating or patching infected systems as well as pro-actively protecting vulnerable uninfected systems from infection.

Containment of malicious intrusions can be executed either at the host or on the network. Hence, worm defense and containment techniques can be broadly classified as host-based or network-based techniques.

Host-based defense techniques typically include:

1. Deployment of system patches [44] [53] against known vulnerabilities and detected malicious code.

2. Activation of host-based filters [21] to block malicious intrusion traffic.

3. Proactively hardening host systems sufficiently to prevent a vulnerability exploit or a successful infection on a single attempt [14]. Such hardening methods include sandboxing, privilege seperation, anomaly detection, system call monitoring [14].

4. Removal of susceptible but uninfected hosts from the network before they become infected [54].

Automated host-based defenses can be successfully deployed in specific scenarios or in specific network zones such as in a server farm or in a demilitarized network zone (DMZ). However, host-based techniques are not very scalable and are unlikely to address worm con- 
tainment in large enterprise networks. Large scale fast spreading worm attacks in particular that traverse several Internet Service Provider (ISP) and enterprise networks would be difficult to contain using host-based techniques. Also, in large networks such as ISP networks that span several geographical regions, network administrators do not typically have any direct control over individual client (customer) machine configuration and therefore cannot depend on such machines for worm containment. Another problem with host-based defense techniques is that a successful compromise of a participating host could subvert the entire defense strategy [77] [56]. A containment technique that ensures fast propagation of the containment action is required. Ideally, such a technique should propagate the containment action at a rate comparable or superior to the rate of propagation of fast spreading malicious worms. While network-based techniques protect a fraction of the target population when executed, host-based techniques protect only a single host at a time. Clearly, for fast containment, network-based techniques should be deployed.

Network-based defense techniques typically implement filters on network devices such as firewalls, routers and intrusion prevention systems to block malicious intrusion traffic [50] [77] or throttle the rate of network traffic flow from suspicious hosts [60] [81]. While rate limiting worm containment schemes can successfully suppress the rapid spread of fast propagating worms, they do not necessarily stop the spread. Also, the criteria for selecting a suspicious traffic flow to rate limit depends heavily on observation of connection success rates [60] or some anomalous network traffic behavior, which are prone to false positives. Hence, there is a concern about false positive rates with rate limiting worm containment schemes. In [50], content filtering based on malicious code signatures was compared to network address blocking techniques and it was shown that if the reaction time between detection and containment can be minimized, address blocking techniques can be more effective. Recent developments in router technology which enables deep packet inspection and content-based filtering [70] has been leveraged for network-based defenses. Further, a collaborative containment architecture should be used to suppress large scale worm attacks [56] [16]. Authors in [56] [16] experimented with the concept of collaborative cellular containment where the network is 
broken into logical cells, each with in-built containment capabilities. Their results show that such a distributed and cooperative approach can greatly improve containment systems. Similarly, the pushback technique [34] employed a collaborative packet dropping technique to suppress distributed denial of service (DDoS) attacks.

In this thesis, we propose a collaborative worm defense scheme triggered by our novel detection and correlation engine. The defense scheme executes network-based containment at the first upstream router (i.e the gateway router) of the network segment or cell under attack. The gateway router of the cell then sends signals to neighbor routers to execute similar containment procedures thus achieving enterprise-wide collaborative containment. We also develop a non-deterministic discrete-time active worm containment model for our proposed collaborative worm defense scheme.

\subsection{Slow Worm Detection}

The propagation behavior of slow scanning worms which enable them blend with normal traffic patterns and evade intrusion detection systems (IDS) that depend on only anomalous network heuristics for detection has made them an interesting research subject. Unlike fast scanning worms, this class of worms propagate and scan the network at rates below detection thresholds of network based intrusion detection systems and host based detection systems that use number of incoming or outgoing connections as a basis for anomalous detection. Such worms are indistinguishable from normal traffic seen on the network, or seen by end host network connections and are difficult to detect. Malicious slow scanning worms therefore pose a serious threat to networks today. Some recent work have attempted to address the problem of detecting slow worms. In [22] a distributed end host detection scheme which uses a dynamic bayesian network model for probabilistic detection was proposed for detection of slow worms. End host detectors alert when the number of outgoing connections to 
unique destination addresses and ports exceed a threshold and share detection information with each other using a gossip messaging protocol to improve false detection rates. The gossip messaging between detectors proposed in [22] was based on the assumption that individual detectors only see a partial picture of the larger network behavior of the worm(s) and therefore are considered weak detectors with high false positive rates. Hence, collaboration through gossips provided a way to remedy this by allowing multiple detectors share information to provide better coverage. In [65] the SWORD (Self-propagating Worm Observation and Rapid Detection) detection system was proposed to detect zero-day worms of different propagation types and speeds. This was achieved by determining whether total number of outgoing worm-like connections from a domain during a sliding window crosses a threshold set based on observation of normal traffic. However, it was acknowledged that if the worm speed is slow enough to cause interspersed traffic throughout a large amount of normal traffic, detection with the SWORD system becomes difficult. In [61] a multiresolution approach for worm detection was proposed to deal with the limitations of simple threshold-based detection methods. Using number of unique destinations contacted as a basis for anomaly detection, the multi-resolution approach used different thresholds during different time windows to detect attacks of different speeds. Faster scanning attacks were detected with smaller time windows while slower attacks were detected with larger time windows.

We distinguish between slow scanning worms and stealth worms [67]. While slow scanning worms randomly scan IP addresses for vulnerable hosts at a low scanning rate, stealth worms are slowly speading but with no peculiar communication patterns [67]. Authors in [67] described an example of a stealth worm in which an attacker has attained a pair of exploits: $E_{s}$, which subverts a popular type of Web server, and $E_{c}$, which subverts a popular type of web client (e.g. a browser). The attacker begins the worm attack on a convenient server or client and then simply waits. If the starting point is a server, then the attacker waits for clients to visit. As each client visits, the subverted server detects whether the client is vulnerable to $E_{c}$. If so, the server infects it, sending along both $E_{c}$ and $E_{s}$. 
As an infected client now surfs other websites, the client inspects whether the servers on those sites are vulnerable to $E_{s}$, and, if so, again infects them, sending along $E_{c}$ and $E_{s}$. In this is example the infection therefore spreads from clients to servers and unto other clients, much as a contagious disease spreads based on the incidental traffic patterns of its hosts. This kind of worm-spread exhibits no peculiar communication pattern and finds easy preys in peer-to-peer networks and is also an interesting research subject [37]. However, in this thesis we focus on the detection of slow scanning worms and not stealth worms.

\subsection{Integrated Detection Techniques}

Worm detection techniques are typically designed based on some unique characteristic(s) of the worm to be detected. Fast scanning worms typically exhibit an abnormally high number of connections or traffic flows which are detectable in the network or on end hosts, while slow scanning worms propagate more stealthily, enabling them to blend with normal traffic patterns and evade intrusion detection systems (IDS) that depend on only anomalous network heuristics for detection. Most proposed techniques for detection of fast scanning worms [2] [77] [60] [15] are unable to detect slow scanning worms. Slow scanning worms are usually indistinguishable from normal traffic seen on the network, or seen by end host network connections and are therefore difficult to detect. However, both types of worms pose serious threats to vulnerable systems and investigating detection systems capable of detecting both types of worms is relevant. Some recent works have attempted to address the challenge of detecting both fast and slow worms. In [1], adaptively adjusting the detection threshold on end host detectors based on observed traffic was proposed as a way to detect both fast and slow worms. A supervised classifier predicts the time-varying distribution of outgoing traffic based on previous observations and this was used for the adjustment. In [61] a multi-resolution approach for worm detection was proposed to deal with the limitations of simple threshold-based detection methods. Using number of unique destinations contacted 
as a basis for anomaly detection, the multi-resolution approach used different thresholds during different time windows to detect attacks of different speeds. Faster scanning attacks were detected with smaller time windows while slower attacks were detected with larger time windows.

We point out that there are two major shortfalls of previously proposed approaches in [1] and [61] to detection of slow scanning and fast scanning worms. First, a common characteristic of both techniques is the use of connection counts and traffic rates as the basis for anomalous detection. This approach inherently carries a high false alarm rate because worms are capable of propagating at rates similar or less than normal traffic rates and therefore can camouflage as normal traffic [27]. Also, anomalous connection counts and traffic rates (whether high or low) do not necessarily confirm the existence of malicious activity. Second, though both techniques demonstrate capability to detect fast scanning and slow scanning intrusions under certain circumstances, they do not address detection of both fast scanning and slow scanning malicious intrusions when they occur concurrently in the same computer network.

Information about vulnerabilities and attempted exploits do not exist in the network layer, hence techniques that use connection counts and traffic rates as the basis for anomalous detection are unable to provide hard evidence of malicious intrusions. In fact, the assumption that malicious attacks necessarily cause anomalous activity in the network in terms of host or network traffic was recently challenged in [27]. On the other hand, host-based Anomaly Intrusion Detection Systems (AIDS) which infer suspicious activity when detector endpoints experience an intrusion that attempts to alter a pre-defined standard state ${ }^{\dagger}$ of the endpoint have been more successful at detecting malicious worm intrusions irrespective of scanning behavior of worms. Typically, such attempts are in the form of anomalous system calls [51], unauthorized or infectious intrusions which cause the host AIDS to trigger an alert.

\footnotetext{
${ }^{\dagger}$ Pre-defined standard states of endpoints are typically determined by established security policies and stan-
} dards. 
Recent work [21] and vendor implementations [68] have recorded success in using host AIDS for detecting unauthorized intrusions. Host AIDS are capable of leveraging large amounts of detailed context about applications and system behavior to effectively detect anomalous host behaviors [64]. The technique adopted in [21] shows that with properly instrumented detection software, host-based intrusion detection is effective and capable of minimizing false positives.

In this thesis, we propose and develop a novel approach to integrated detection of both slow scanning stealthy worms and fast scanning malicious worms that is distinct from previously proposed approaches. First, our proposed detection approach uses an optimized correlation technique based on a decision level multi-sensor data fusion technique to combine malicious intrusion evidence provided by distributed host-based anomaly intrusion detectors. This inherently reduces false alarm rates and optimizes detection accuracy. Second, our detection approach takes into consideration the real possibility that slow scanning stealthy worms can occur concurrently with faster propagating malicious worms in computer networks. This particularly exacerbates the difficulty in detecting slow worms due to interference from other traffic flows scanning at faster rates.

\subsection{Multi-sensor Data Fusion - Introduction}

In [39], data fusion is described as a multifaceted process dealing with the automatic detection, correlation and combination of data and information from single or multiple sources to achieve a refined assessment of situations and threats. Multi-sensor data fusion is concerned with combining data from multiple sensors in order to make inferences about events, activities or situations that may not be possible with a single sensor alone [31]. Typically, multiple sensors are used to enable more accurate data to be gathered about events present in the observation space of the sensors. Multi-sensor data fusion applications exist in several 
areas of study such as artificial intelligence [30], signal processing, statistical estimation [75], pattern recognition [43] etc. where combination of data is required. It has been described as a technology that holds promise in the development of highly reliable intrusion detection and next generation security-decision systems to combat multiple complex threats [12] [11].

We are interested in decision-level identity fusion, an aspect of multi-sensor data fusion that involves combining decisions from individual sensors to achieve a more accurate and refined joint or fused decision on the particular observation of interest. Techniques used for decision-level multi-sensor data fusion include classical inference, Bayesian inference, Dempster-Shafer method, and the Generalized Evidence Processing theory. We describe decision-level multi-sensor data fusion techniques in more detail in Chapter four.

In this thesis, we apply the Generalized Evidence Processing (GEP) theory to the detection of malicious worms in computer networks. The GEP theory offers an optimized and flexible decision-level identity fusion technique for combining individual sensor decisions and is known to have inherent advantages over the other decision-level multi-sensor data fusion techniques. These are discussed in more detail in Chapter four. 


\section{Chapter 4}

\section{Background}

This thesis investigates the problem of detecting and containing worms and malicious intrusions in computer networks. In this chapter, we review multi-sensor data fusion, a wellknown approach to making decisions based on distributed sensing and data combination. Data fusion techniques such as Bayesian inference and the Dempster-Shafer's evidential theory are briefly discussed. We then discuss in detail the Generalized Evidence Processing (GEP) theory technique and its application to detection of malicious intrusions. We also highlight the advantages of the GEP theory technique over the other techniques. This thesis focuses on using a detection and correlation engine based on the Generalized Evidence Processing theory for detecting malicious worms in computer networks.

\subsection{Decision Level Multi-sensor Data Fusion}

Multi-sensor data fusion is the process of combining data from multiple sensors to perform inferences or to improve the accuracy of inferences that may not be possible with only a single sensor. This thesis is concerned with the use of multi-sensor data fusion for detection 
of worm attacks and malicious intrusions in computer networks. In particular, it focuses on decision-level data fusion where a fusion center processes identity declarations or decisions from multiple sensors to achieve a joint declaration of identify also referred to as a fused decision. With decision-level fusion, multiple sensors participate in monitoring an event or activity of interest and perform individual processing and identity declaration concerning the observed event. The individual sensor identity declarations are then combined or fused in a fusion center to achieve a joint declaration that more acurately identifies the event of interest. Typically, individual sensor identity declarations can be either a hard decision concerning the event which provides only a single declaration of identity or a soft decision which provides multiple declarations of identity with an associated probability [31] *. With decision-level fusion, techniques used for fusing individual sensor declarations include classical inference, Bayesian inference, Dempster-Shafer evidential theory, Generalized Evidence Processing theory and heuristic approaches such as voting methods.

In this thesis, we pay particular attention to the Generalized Evidence Processing theory technique and develop a detection and correlation engine based on the technique for detecting malicious worms in computer networks. In the next section, we briefly describe the Bayesian inference and the Dempster-Shafer evidential theory.

\subsubsection{Bayesian Inference}

Bayesian inference is a probability-based decision level data fusion technique that determines the validity of a proposed hypothesis concerning an event versus an alternative hypothesis based on Baye's rule [13]. With Bayesian inference, the fusion center updates the probabilities of alternative hypotheses based on observations or declarations from multiple sensors. The technique updates the likelihood of a hypothesis given a previous likelihood estimate and additional sensor observations or declarations. This provides a relationship between the

\footnotetext{
* Soft decisions give a notion of uncertainty about the true identity of the observed event.
} 
a priori probability of the hypothesis, and the a posteriori probability of the hypothesis.

Assume $H_{1}, H_{2}, \ldots H_{k}$ are mutually exclusive hypotheses that describe or identify an event $X$ which has just occurred, then with Bayesian inference,

$$
P\left(H_{i} \mid X\right)=\frac{P\left(X \mid H_{i}\right) P\left(H_{i}\right)}{\sum_{i=1}^{k} P\left(X \mid H_{i}\right) P\left(H_{i}\right)}
$$

and

$$
\sum_{i=1}^{k} P\left(H_{i}\right)=1
$$

where $P\left(H_{i}\right)$ is the a priori probability of hypothesis $H_{i}$ being true (before observing the event or evidence $X), P\left(X \mid H_{i}\right)$ is the probability of observing evidence $X$ given that $H_{i}$ is true. $P\left(X \mid H_{i}\right)$ is also called a likelihood function when it is considered as a function of $H_{i}$ for fixed $X . P\left(H_{i} \mid X\right)$ is the a posteriori probability of hypothesis $H_{i}$ being true given that evidence $X$ is available (or event $X$ has occured).

For detection of malicious intrusions two hypotheses are typically of interest, the hypothesis that an observed intrusion is malicious and the hypothesis that the intrusion is benign. These are mutually exclusive hypotheses and are therefore suited for investigation using Bayesian inference. In a multi-sensor detection system, observations or declarations from distributed sensors (or detectors) provide evidence used to update the probabilities of each hypotheses until a probabilistic decision is made based on some stopping conditions. While Bayesian inference provides a tractable approach to data fusion, it has been criticized for lack of flexibility when evidence provided by detector observations do not necessarily support hard decisions. In other words, capability of sensors to make hard decisions is required for the Bayesian inference technique to be usable. Other limitations of the technique include the requirement for mutual exclusivity between competing hypotheses [63] [31] and the fact 
that there can be difficulty in defining a priori probabilities and likelihood functions for the alternative hypotheses.

\subsubsection{Dempster-Shafer Evidential Theory}

Similar to the Bayesian inference, the Dempster-Shafer technique is a decision level data fusion technique. Whereas the Bayesian theory requires probabilities for each hypothesis of interest, the Dempster-Shafer theory uses a concept of belief functions with associated weighting for particular propositions. For detection of malicious intrusions, weighting or mass values from individual detectors are fused using the Dempster-Shafer rule of combination to compute a combined mass value for a particular proposition [82] [18]. This quantifies the credibility (measure of belief) of the proposition and its plausibility (lack of evidence refuting the proposition). Proponents of the Dempster-Shafer theory argue that the Dempster-Shafer technique offers unique advantages for a number of reasons. It was explained in [82] that the capability to assign uncertainty or ignorance to Dempster-Shafer propositions makes it more applicable to problems that would otherwise seem intractable. Since a priori knowledge is not required for the Dempster-Shafer technique unlike Bayesian inference, authors in [18] affirmed that the Dempster-Shafer technique is potentially more suitable for anomaly detection of previously unseen events or situations. Some of the disadvantages of the Dempster-Shafer method include inability to use a priori probabilities when they are known, and counter-intuitive results that are sometimes generated when support for conflicting propositions is significant [39]. Also, the Dempster-Shafer theory does not natively offer a decision logic to facilitate arriving at a fused decision even though it provides evidence supporting the hypotheses or propositions being considered [39]. In addition, the computational complexity of the technique increases exponentially with the number of propositions under consideration [18]. 


\subsubsection{Generalized Evidence Processing Theory}

Some of the highlighted limitations of the Bayesian inference or decision processes include inability to deal with both non-mutually exclusive multiple hypotheses and uncertainty [39] [31]. The Generalized Evidence Processing (GEP) theory [72] [73], also a decision level data fusion technique extends the Bayesian inference framework to deal with these limitations. As an example, let $H_{0}$ and $H_{1}$ be the two hypotheses under testing. The events associated with the probability space can be attributed to the two hypotheses $H_{0}$ and $H_{1}$ with probabilities $P\left(H_{0}\right) \geq 0$ and $P\left(H_{1}\right) \geq 0$ respectively, where $P\left(H_{0}\right)+P\left(H_{1}\right) \leq 1$. Let $D_{0}, D_{1}$, and $D_{2}$ be the decisions which correspond to the propositions " $H_{0}$ is true", " $H_{1}$ is true" and " $H_{0}$ or $H_{1}$ is true" ${ }^{\dagger}$ respectively. With Bayesian inference where $H_{0}$ and $H_{1}$ are mutually exclusive events, the probability associated with $D_{2}$ is equivalent to:

$$
P\left(D_{2}\right)=P\left(H_{0} \cup H_{1}\right)=P\left(H_{0}\right)+P\left(H_{1}\right)=1
$$

This shows an inability to account for non-mutually exclusive events and uncertainty (or indecision) within the Bayesian inference framework. The Dempster-Shafer technique also has shortcomings as explained earlier in Section 4.1.2. The GEP theory is a unified evidence combining theory which accounts for indecision and combines evidence that supports nonmutually exclusive propositions to arrive at a decision by minimizing a cummulative risk function. Hence, the GEP theory enjoys the merits of both the Bayesian and the DempsterShafer techniques and avoids their shortcomings [39]. In a distributed multi-sensor system with $N$ sensors (see Fig. 4.1), let $\mathbf{Z}$ be the observation (data) space which results in individual local decisions on the sensors. We represent $\mathbf{Z}$ as $\mathbf{Z}=\left\{\underline{z}: \underline{z}=\left(z_{1}, z_{2}, . ., z_{N}\right), z_{i}=0,1,2\right\}$ where 0 implies a "benign observation", 1 implies a "malicious observation" and 2 implies an "uncertainty" about the nature of the observation. Also, for each sensor let two hypotheses $H_{1}$ and $H_{0}$ be considered, where $H_{1}$ is the hypothesis that the observation is malicious and $H_{0}$ is the hypothesis that the observation is benign. Each local sensor observation results in

\footnotetext{
${ }^{\dagger}$ Decision $D_{2}$ therefore represents an indecision about the true nature of the hypothesis.
} 


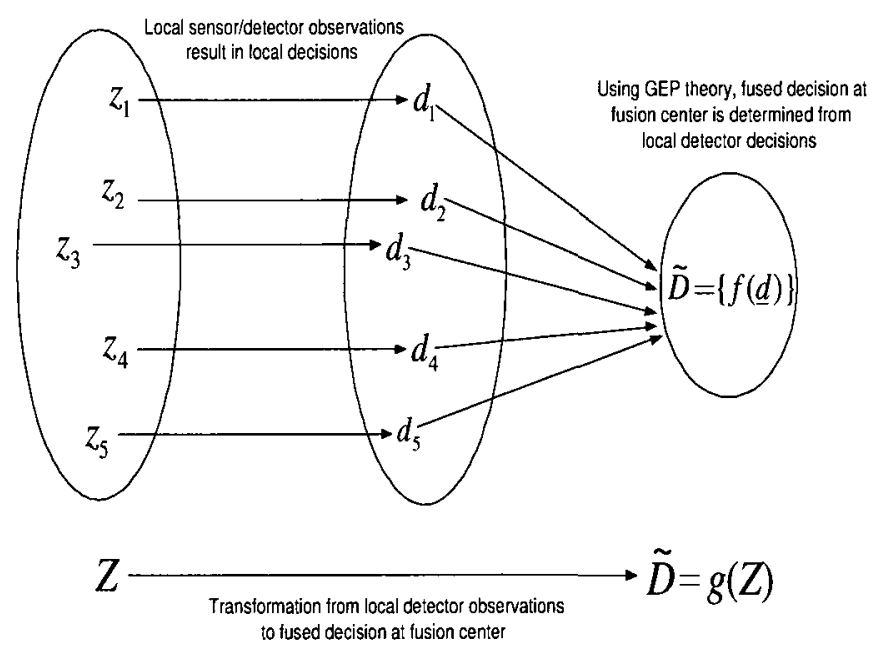

Figure 4.1: Transformation from $N$ local detector observations to a fused decision. $N=5$.

a local sensor decision (see Fig. 4.1). Hence, the vector of observations $\underline{z}$ results in a vector of local decisions $\left\{\underline{d}: \underline{d}=\left(d_{1}, d_{2}, . ., d_{N}\right), d_{i}=0,1,2\right\}$, where 0,1 , and 2 are the individual local decisions which correspond to the propositions " $H_{0}$ is true", " $H_{1}$ is true" and " $H_{0}$ or $H_{1}$ is true" (i.e. an indecision). Using the GEP theory, the local sensor decisions are combined at a fusion center to arrive at a fused decision that minimizes a cummulative risk function. As depicted in Fig. 4.1, let $g(\mathbf{Z})$ be the transformation from the observation space $\mathbf{Z}$ into the fused decision space $\tilde{\mathbf{D}}=\left\{\mathbf{D}=f(\underline{d}): \underline{d}=\left(d_{1}, d_{2}, . ., d_{N}\right), d_{i}=0,1,2\right\}$ such that

$$
\tilde{\mathbf{D}}=g(\mathbf{Z}), \quad \tilde{\mathbf{D}}=\{0,1,2\} \quad \text { and } \quad \mathbf{D}=0,1,2
$$

where $\mathbf{D}=0,1,2$ are the fused decisions that " $H_{0}$ is true", " $H_{1}$ is true" and " $H_{0}$ or $H_{1}$ is true" respectively.

In practical worm detection systems, distributed detectors in a network can make observations of malicious intrusions in the network and report their individual decisions to a central processor (such as a gateway router). The transformation $g(\mathbf{Z})$ corresponds to the function of a correlation algorithm running on the gateway router that takes as input the individual local decisions of the detectors and outputs a fused decision. 
Following the GEP theory [72] [73], let $C_{a b}$ be the cost associated with a fused decision $a$ (that is in set $\tilde{\mathbf{D}}$ at the fusion center) when hypothesis $H_{b}$ is true, where $0 \leq C_{a b} \leq 1$. We assume that there is no penalty for a correct fused decision, hence the associated cost for a correct decision is zero (i.e. $C_{00}=C_{11}=0$ ). We now determine the decision rules which ensure the fused decision (4.3) made at the fusion center minimizes the cummulative decision risk $\mathbf{R}$. As shown in [72] the cummulative risk $\mathbf{R}$ can be expressed as:

$$
\mathbf{R}=\sum_{a} \sum_{b} C_{a b} P\left(H_{b}\right) \int_{\mathbf{D}=a} d P\left(\underline{d} \mid H_{b}\right) ; b=0,1 \text { and } a=0,1,2
$$

where $\mathbf{D}=0,1,2$ are the fused decisions that " $H_{0}$ is true", " $H_{1}$ is true" and " $H_{0}$ or $H_{1}$ is true" respectively which occupy the fused decision space. Solving,

$$
\begin{aligned}
\mathbf{R} & =\int_{\mathbf{D}=0}\left[P\left(H_{0}\right) C_{00} d P\left(\underline{d} \mid H_{0}\right)+P\left(H_{1}\right) C_{01} d P\left(\underline{d} \mid H_{1}\right)\right] \\
& +\int_{\mathbf{D}=1}\left[P\left(H_{0}\right) C_{10} d P\left(\underline{d} \mid H_{0}\right)+P\left(H_{1}\right) C_{11} d P\left(\underline{d} \mid H_{1}\right)\right] \\
& +\int_{\mathbf{D}=2}\left[P\left(H_{0}\right) C_{20} d P\left(\underline{d} \mid H_{0}\right)+P\left(H_{1}\right) C_{21} d P\left(\underline{d} \mid H_{1}\right)\right]
\end{aligned}
$$

$\mathbf{R}$ is minimized if the fusion decision rule assigns $\mathbf{D}$ (the fused decision) to a region $(\mathbf{D}=0$, $\mathbf{D}=1$ or $\mathbf{D}=2$ ) that results in the least integrand under the three integrals. Since $\underline{d}$ is a vector with discrete components, we can write the fusion decision rules as follows:

$$
\begin{aligned}
& P\left(H_{0}\right) C_{00} P\left(\underline{d} \mid H_{0}\right)+P\left(H_{1}\right) C_{01} P\left(\underline{d} \mid H_{1}\right) \underset{\mathbf{D}=0 \text { or } \mathbf{D}=2}{\mathbf{D}=1} \stackrel{\text { or }}{\mathbf{D}=2} P\left(H_{0}\right) C_{10} P\left(\underline{d} \mid H_{0}\right)+P\left(H_{1}\right) C_{11} P\left(\underline{d} \mid H_{1}\right) \\
& P\left(H_{0}\right) C_{00} P\left(\underline{d} \mid H_{0}\right)+P\left(H_{1}\right) C_{01} P\left(\underline{d} \mid H_{1}\right) \underset{\mathbf{D}=0 \text { or } \mathbf{D}=1}{\mathbf{D}=2 \text { or } \mathbf{D}=1} P\left(H_{0}\right) C_{20} P\left(\underline{d} \mid H_{0}\right)+P\left(H_{1}\right) C_{21} P\left(\underline{d} \mid H_{1}\right) \\
& P\left(H_{0}\right) C_{20} P\left(\underline{d} \mid H_{0}\right)+P\left(H_{1}\right) C_{21} P\left(\underline{d} \mid H_{1}\right) \underset{\mathbf{D}=2 \text { or } \mathbf{D}=0}{\stackrel{\mathbf{D}=1}{\text { or }} \mathbf{D}=0} P\left(H_{0}\right) C_{10} P\left(\underline{d} \mid H_{0}\right)+P\left(H_{1}\right) C_{11} P\left(\underline{d} \mid H_{1}\right)
\end{aligned}
$$


where,

$$
L \stackrel{d_{x}}{\gtrless} R
$$

implies that decision $d_{x}$ is made if $L>R$, otherwise decision $d_{y}$ is made.

Dividing both sides by $P\left(\underline{d} \mid H_{0}\right)$ and defining $\Lambda(\underline{d})=\frac{P\left(\underline{d} \mid H_{1}\right)}{P\left(\underline{d} \mid H_{0}\right)}$ the decision rules become:

$$
\begin{aligned}
& {\left[C_{01}-C_{11}\right] \Lambda(\underline{d}) \underset{\mathbf{D}=0 \text { or } \mathbf{D}=2}{\mathbf{D}=1 \text { or } \mathbf{D}=2} \frac{P\left(H_{0}\right)}{P\left(H_{1}\right)}\left[C_{10}-C_{00}\right]} \\
& {\left[C_{01}-C_{21}\right] \Lambda(\underline{d}) \underset{\mathbf{D}=0 \text { or } \mathbf{D}=1}{\mathbf{D}=2 \text { or } \mathbf{D}=1} \frac{P\left(H_{0}\right)}{P\left(H_{1}\right)}\left[C_{20}-C_{00}\right]} \\
& {\left[C_{21}-C_{11}\right] \Lambda(\underline{d}) \underset{\mathbf{D}=1}{\stackrel{\text { or }}{\gtrless} \mathbf{D}=0} \frac{P\left(H_{0}\right)}{P\left(H_{1}\right)}\left[C_{10}-C_{20}\right]}
\end{aligned}
$$

Solving,

$$
\begin{aligned}
& \Lambda(\underline{d}) \underset{\mathbf{D}=0}{\mathbf{D}=1} \underset{\text { or }}{\stackrel{\text { or }}{ } \mathbf{D}=2} \mathbf{D} \frac{P\left(H_{0}\right)}{P\left(H_{1}\right)} \frac{C_{10}}{C_{01}} \\
& \Lambda(\underline{d}) \underset{\mathbf{D}=0}{\mathbf{D}=2} \underset{\text { or }}{\stackrel{\text { or }}{\gtrless} \mathbf{D}=1} \frac{P\left(H_{0}\right)}{P\left(H_{1}\right)} \frac{C_{20}}{C_{01}-C_{21}} \\
& \Lambda(\underline{d}) \underset{\mathbf{D}=2 \text { or }}{\mathbf{D}=1} \underset{\text { or }}{\mathbf{D}=0} \frac{P\left(H_{0}\right)}{P\left(H_{1}\right)} \frac{C_{10}-C_{20}}{C_{21}}
\end{aligned}
$$

According to equation (4.5), (4.6), (4.7), the fusion decision rules depend on the values of the $C_{a b}$ costs and a priori probabilities $P\left(H_{1}\right)$ and $P\left(H_{0}\right)$ of the two hypotheses, $H_{1}$ and $H_{0}$ respectively. We assume that the a priori probabilities are known and we are interested in estimating $\Lambda(\underline{d})$. 
For malicious worm detection, $C_{10}$ and $C_{01}$ are the costs associated with a decision that results in a false positive and a false negative respectively. For worm detection systems without a bias for false positives or false negatives, cost values $C_{10}=C_{01}$ is appropriate to ensure the same penalty for decisions that result in either false positives or false negatives.

Equation (4.5), (4.6), (4.7) also show that the GEP framework can make use of the a priori probabilities of both hypothesis $H_{1}$ and $H_{0}$ if they are known. When they are not known, we assume that $P\left(H_{1}\right)=P\left(H_{0}\right)$ thus nullifying the impact of a priori probabilities on the fusion decision rules in (4.5), (4.6), (4.7). Also, note that the GEP decision process breaks down to a binary decision process if indecision is not considered. In this case, only the decision rule (4.5) applies since $C_{21}$ and $C_{20}$ become undefined.

To illustrate an application of the decision rules, we consider different possible cases as was done in [72]. We assume a priori probabilities of both hypothesis $H_{1}$ and $H_{0}$ are unknown, hence $P\left(H_{1}\right)=P\left(H_{0}\right)$, and that the cost of an incorrect decision is greater than the cost associated with an indecision (i.e. $C_{10}>C_{20}, C_{01}>C_{21}$ ).

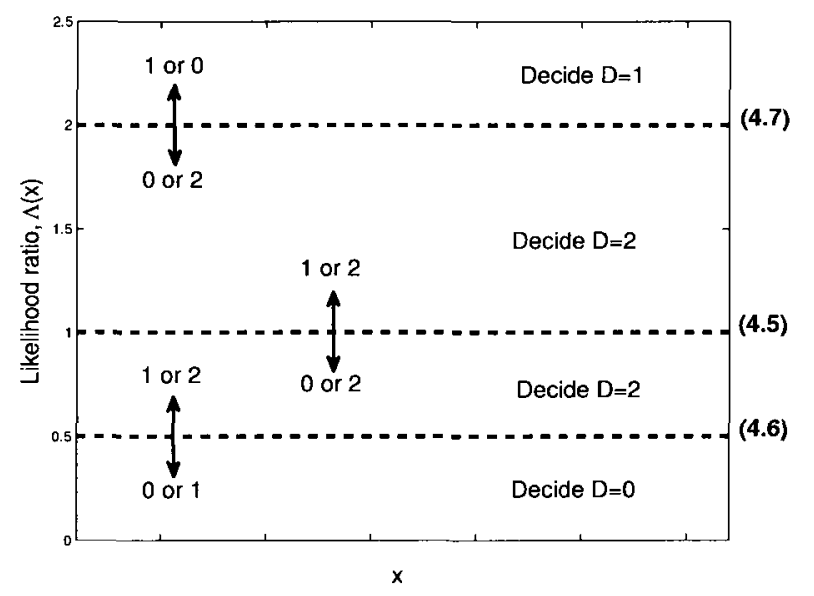

Figure 4.2: Case 1: The indecision region lies between the two definite decision regions. 


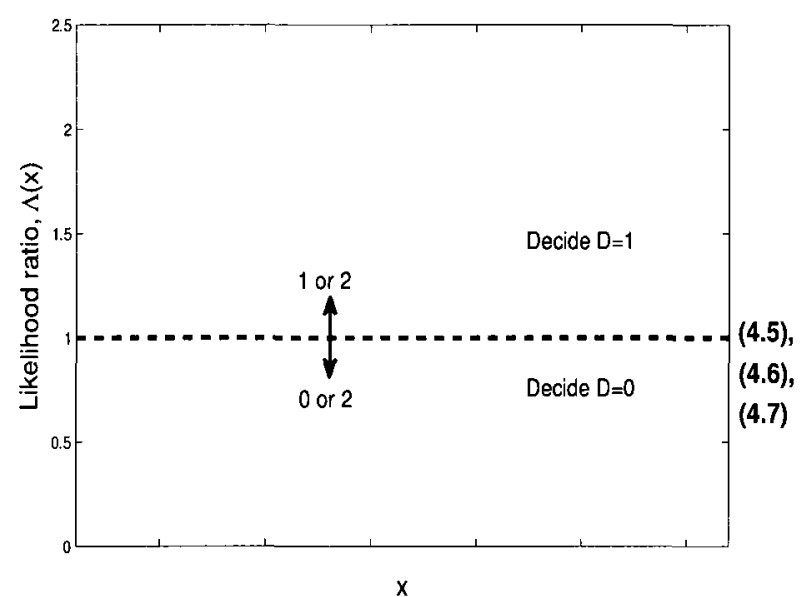

Figure 4.3: Case 2: The indecision region is completely eliminated.

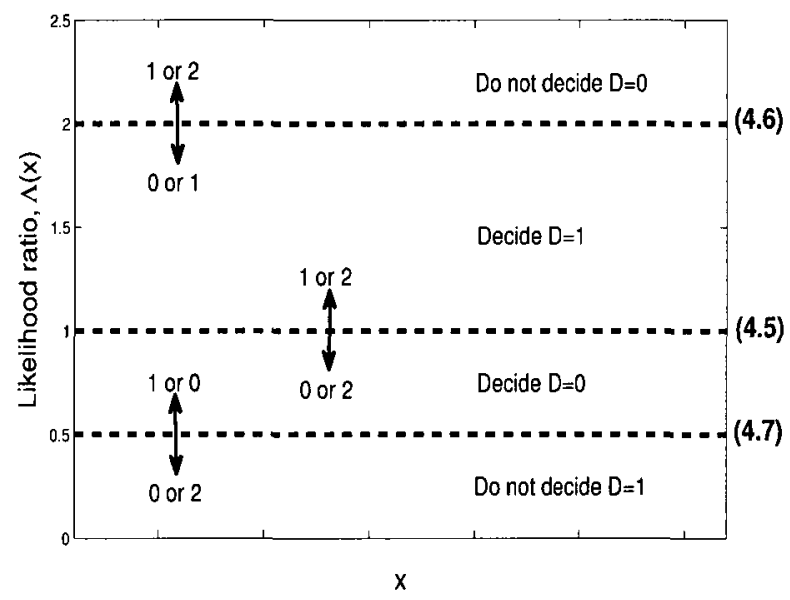

Figure 4.4: Case 3: The definite decision regions lies between two indecision regions.

Case 1: $C_{00}=C_{11}=0, C_{10}>2 * C_{20}, C_{01}>2 * C_{21}$

Let $C_{10}=C_{01}=1, C_{20}=C_{21}=\frac{1}{3}$. Hence, $\frac{C_{10}}{C_{01}}=1, \frac{C_{20}}{C_{01}-C_{21}}=0.5$, and $\frac{C_{10}-C_{20}}{C_{21}}=2$. Equation (4.5), (4.6), (4.7) are used to generate the partition in Fig. 4.2. In this case, Fig. 4.2 shows that the indecision region lies between the two definite decision regions. This case is applicable to practical detection systems that are not always capable of providing 
evidence to support definite decisions, hence the option of indecision is provided.

Case 2: $C_{00}=C_{11}=0, C_{10}=2 * C_{20}, C_{01}=2 * C_{21}$

Let $C_{10}=C_{01}=1, C_{20}=C_{21}=0.5$. Hence, $\frac{C_{10}}{C_{01}}=\frac{C_{20}}{C_{01}-C_{21}}=\frac{C_{10}-C_{20}}{C_{21}}=1$. All three thresholds have the same values and the indecision region is non-existent as shown in Fig. 4.3. In this case the decision process corresponds to a standard binary decision process. This case is applicable if the detection system is capable of always providing hard evidence sufficient to support a decision or if the system is not capable of dealing with indecision.

Case 3: $C_{00}=C_{11}=0, C_{10}<2 * C_{20}, C_{01}<2 * C_{21}$

Let $C_{10}=C_{01}=1, C_{20}=C_{21}=\frac{2}{3}$. Hence, $\frac{C_{10}}{C_{01}}=1, \frac{C_{20}}{C_{01}-C_{21}}=2$, and $\frac{C_{10}-C_{20}}{C_{21}}=0.5$. In this case, Fig. 4.4 shows that the two definite decision regions lie between two indecision regions, an exact opposite of Case 1. Case 3 represents a detection system that exhibits a standard binary decision process within a likelihood ratio bound (in this case $0.5<\Lambda(x)<2$ ). Beyond the bound, the detection system is incapable of making a definite decision.

Practical detection systems are more suited to Case 1 and Case 2. 


\section{Chapter 5}

\section{EDANC Detection Technique for Fast Scanning Worms}

\subsection{Introduction}

Anomaly-based intrusion detection has emerged as a mechanism for defending against advanced malicious intrusions that are either previously unknown or are capable of bypassing traditional signature-based detection schemes [32] [44]. Anomaly-based Intrusion Detection Systems (AIDS) can be classified as network AIDS or host AIDS depending on where the anomalous behavior is detected. Network AIDS infer suspicious activity in a network by detecting anomalous network traffic patterns [79] [10] and trends [84]. While this approach can effectively detect fast spreading worms under favorable conditions, it cannot reliably detect malicious intrusions that do not cause anomalous network traffic patterns [21]. Hostbased AIDS have been more successful at detecting malicious worm intrusions irrespective of scanning behavior of worms since a detection alert is generated when an intrusion attempts 
to alter the standard state ${ }^{*}$ of the endpoint. Typically, such attempts are in the form of anomalous system calls [51] or unauthorized intrusions which cause the host AIDS to trigger an alert. This aligns with the argument in [21] that since end hosts running vulnerable software are the targets of malicious code attacks, they ought to be the point of detection. Recent work [21] [2] [5] and vendor implementations [68] have recorded success in using host AIDS for detecting unauthorized intrusions. Host AIDS are capable of leveraging large amounts of detailed context about applications and system behavior to effectively detect anomalous host behaviors [64]. The technique adopted in [21] shows that with properly instrumented detection software, host-based intrusion detection is effective and capable of eliminating false positives.

In this chapter, use of the Generalized Evidence Processing (GEP) theory, a multi-sensor data fusion technique, for combining intrusion detection evidence provided by distributed host-based intrusion detectors [72] [73] is introduced. There has been previous attempts to use two major evidence combining theories for intrusion detection - the Bayesian theory and the Dempster-Shafer theory [47] [63]. Proponents of the Bayesian theory criticize the Dempster-Shafer theory for lack of rigorousness in the axiomatic definition of evidence and the inability to use a priori probabilities when they are known [72] [39]. Also, the DempsterShafer theory does not natively offer a decision logic to facilitate arriving at a fused decision even though it provides evidence supporting the hypotheses or propositions being considered [39]. On the other hand, proponents of the Dempster-Shafer theory criticize the Bayesian theory for lack of flexibility when it comes to fuzzy decisions where the evidence might not support hard decisions, difficulty in defining a priori probabilities and likelihood functions, as well as the mutual exclusivity requirement for competing hypotheses [63] [31]. The GEP theory unifies both theories in a generalized framework and combines their advantages [72] [31]. With GEP, the evidence collected by the host detectors determines the probability mass associated with a decision under a hypothesis. The probability mass assignments may

*Pre-defined standard states of endpoints are typically determined by established security policies and standards. 
be based on the Bayesian likelihood function or correspond to the belief functions used in the Dempster-Shafer evidential theory. The probability mass assignments associated with each host detector decision is combined at a fusion center to arrive at an improved fused decision by minimizing a decision risk function.

This thesis proposes the EDANC scheme which uses host-based anomaly intrusion detectors as sensors in a target cell (or network segment) and runs a GEP-based correlation algorithm on their gateway router, which acts as a fusion center to achieve an improved detection decision. Based on that decision, the gateway router communicates with other routers in the network to achieve collaborative worm containment in the network. With the EDANC scheme, the host detectors do not participate in normal traffic transactions but function solely as anomaly detectors in the target cell. Their role is to make local decisions (malicious or benign) concerning detected intrusions and communicate that decision to their gateway router. There is evidence of the effectiveness of host-based anomaly detection techniques in detecting unauthorized and malicious host intrusions with minimal false alarms [21] [36]. The EDANC technique is unique because it leverages probabilistic evidence and local decisions from distributed host-based anomaly detectors about unauthorized intrusions, as well as an optimized correlation of the local decisions and evidence based on the GEP theory to achieve network-based collaborative containment of fast spreading worms. Though the GEP theory can be generalized to cover detector indecision, the implementation of our proposed EDANC detection scheme does not consider indecision in this thesis. We focus on detectors that are capable of making decisions and leave detector indecision for future work.

The main contributions in this chapter are:

$\dagger$ We propose the EDANC scheme which uses an optimized intrusion detection technique based on the Generalized Evidence Processing (GEP) theory. The GEP theory is a sensor integration and decision level data fusion technique known to have advantages over the two major evidence combining theories that have dominated the field of 


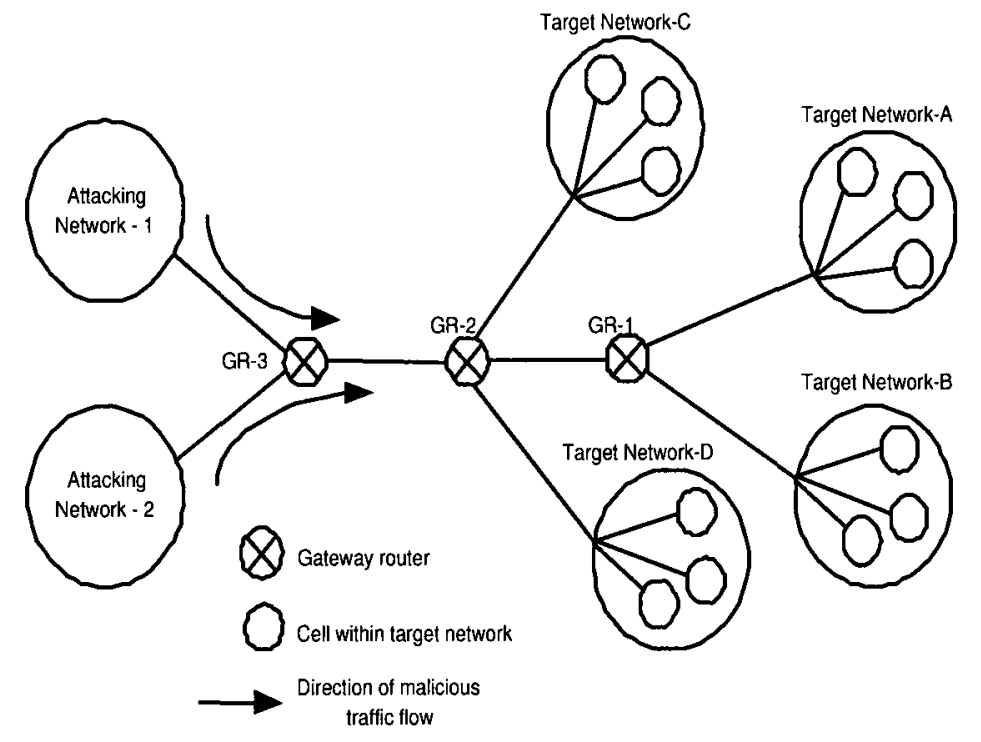

Figure 5.1: Typical worm attack on multiple networks

distributed evidence processing - the Bayesian theory and the Dempster-Shafer theory.

$\dagger$ We present an analysis of EDANC detection interval for a fast scanning worm.

$\dagger$ Experimenting on a live test-bed we evaluate the EDANC scheme and show that the results obtained concur with analytical results. The experiments also show that our GEP-based EDANC detection technique is a viable intrusion detection technique and exhibits much better accuracy than the use of a single host detector for detection.

\subsection{The EDANC Scheme}

Fig. 5.1 depicts a typical attack scenario in which single or multiple attackers in Network1 and Network-2 launch fast scanning worm attacks on several enterprise networks (Target Network-A, Target Network-B, Target Network-C, Target Network-D). Typically, welldesigned enterprise networks are logically subdivided into cells or network zones as shown in Fig. 5.1. 


\subsubsection{Detection Technique}

The EDANC scheme uses host based anomaly detection software running on detector endpoints (DEs) for detecting malicious intrusions into the network. In a large enterprise network, a number of DEs located within distributed cells detect and respond to intrusion attempts targeted at the cells. Individual DEs are capable of performing real-time monitoring and recording of profiles of all network traffic originated from outside their cell and targeted at the DEs. We define a profile as a 4-tuple consisting of srcIP, dstport, proto, payload. srcIP is the source IP address in the IP header of packets captured by the DE, dstport is the target port, proto is the transport layer protocol used, and payload is the signature of the exploit in the payload of the IP packet. When the host AIDS ${ }^{\dagger}$ running on a detector endpoint (DE) detects an unauthorized intrusion associated with a particular traffic flow the following occurs:

1. The DE immediately starts real-time recording of profiles of all network traffic originated from outside its cell and targeted at the $\mathrm{DE}$ for a pre-set capture interval. The DE capture interval is the fast worm detection window with duration $t_{f}$.

2. The DE also immediately sends an alert to other participating DEs within the cell. DEs communicate only with other DEs.

3. When the alert is received, the DEs within the target cell also start real-time recording of profiles for all network traffic originated from outside their cell and targeted at the DEs for the pre-set capture interval.

4. For each traffic profile $i$ detected in the target cell by a DE, two hypotheses $H_{1}$ and $H_{0}$ are considered, where $H_{1}$ is the hypothesis that the traffic profile $i$ is malicious and $H_{0}$ is the hypothesis that the traffic profile $i$ is benign. For the profile $i$, let $d_{q}^{i}$ be the individual local decision by the $q^{\text {th }} \mathrm{DE}$ based on observed intrusion attempts. $d_{q}^{i}=1$ if

\footnotetext{
${ }^{\dagger}$ Emulated using a detector agent (DA) that constantly monitored the directory structure and directory content on the DE as well as the file transfer logs on the DE.
} 
$H_{1}$ is decided and $d_{q}^{i}=0$ if $H_{0}$ is decided. We assume the anomaly detection software running on a DE is capable of making such a decision. For this work, we considered a binary local detection outcome which did not include indecision. However, the GEP theory is capable of dealing with indecision as explained earlier.

5. At the end of the detection window, the DEs in the cell transfer their records and local decisions to their upstream gateway router and continue monitoring for unauthorized intrusions. For a target cell with $m$ DEs, let $\underline{d}^{i}=\left(d_{1}^{i}, d_{2}^{i}, . ., d_{m_{i}}^{i}\right)$ be the vector of individual DE decisions on traffic profile $i$.

The fundamental role of the DEs is to make independent local decisions (malicious or benign) concerning detected intrusions and communicate that decision to their gateway router.

\subsubsection{Correlation Technique}

The upstream gateway router receives the records and local decisions transfered from the DEs in the target cell. The gateway router runs a correlation engine based on the Generalized Evidence Processing (GEP) theory data fusion technique to determine the traffic profile(s) associated with the detected malicious or unauthorized intrusion(s). Multiple correlation processes can run on the gateway router simultaneously.

\subsubsection{Correlation Engine (based on GEP Theory)}

At the gateway router, we are interested in using collected DE local decisions and records in making an optimized fused decision which minimizes the cummulative decision risk of making a decision. For each traffic profile $i$ with associated records and local decisions received from the DEs, two hypotheses $H_{1}$ and $H_{0}$ are considered at the gateway router, where $H_{1}$ is the 
hypothesis that the traffic profile $i$ is malicious and $H_{0}$ is the hypothesis that the traffic profile $i$ is benign. In general, the a priori probabilities of the two hypothesis $P_{i}\left(H_{1}\right)$ and $P_{i}\left(H_{0}\right)$ can be estimated using historical data or experience. However, without loss of generality, we assume that $P_{i}\left(H_{1}\right)$ and $P_{i}\left(H_{0}\right)$ for each profile $i$ are the same. Hence, the GEP optimized decision criteria at the fusion centre (the gateway router) which minimizes the cummulative decision risk can be expressed using the following likelihood ratio rule (derived from (4.5)):

$$
\Lambda\left(\underline{d}^{i}\right)=\frac{P\left(\underline{d}^{i} \mid H_{1}\right)}{P\left(\underline{d}^{i} \mid H_{0}\right)} \underset{\mathbf{D}=0}{\stackrel{\mathrm{D}=1}{\gtrless}} \frac{C_{10}}{C_{01}}=\gamma
$$

where $C_{10}$ and $C_{01}$ are the costs of a decision that results in a false positive and a false negative respectively. Using notations derived in Section $4.1 .3, \mathbf{D}=1$ is the fused decision that traffic profile $i$ is a malicious traffic profile while $\mathbf{D}=\mathbf{0}$ is the fused decision that traffic profile $i$ is a benign traffic profile. The choice of $C_{10}$ and $C_{01}$ is system design driven and has an impact on the behavior of our algorithm as we show in our experiments. Since the goal of our EDANC technique is to automatically detect fast malicious intrusions and generate a response, we consider false positives more severe than false negatives because false positives are likely to cause self-inflicted service disruptions which will degrade network reliability. Therefore, a $\gamma=\frac{C_{10}}{C_{01}}$ value of 2.0 was used in our experiments with fast worm detection to ensure that a greater cost or penalty was associated with a decision that results in a false positive than a decision that results in a false negative. Previous works on worm detection and containment have made similar assumptions concerning fast worms and false positives [77].

In our implementation we do not consider indecision, hence the GEP decision process breaks down to a binary decision process. Though the GEP theory framework can support indecision, we reserve that for future work and focus on fused decisions that are definite. This corresponds to Case 2 in Section 4.1.3.

To express (5.1) in more practical terms, let $p_{d q}$ denote the detection probability and $p_{f q}$ de- 


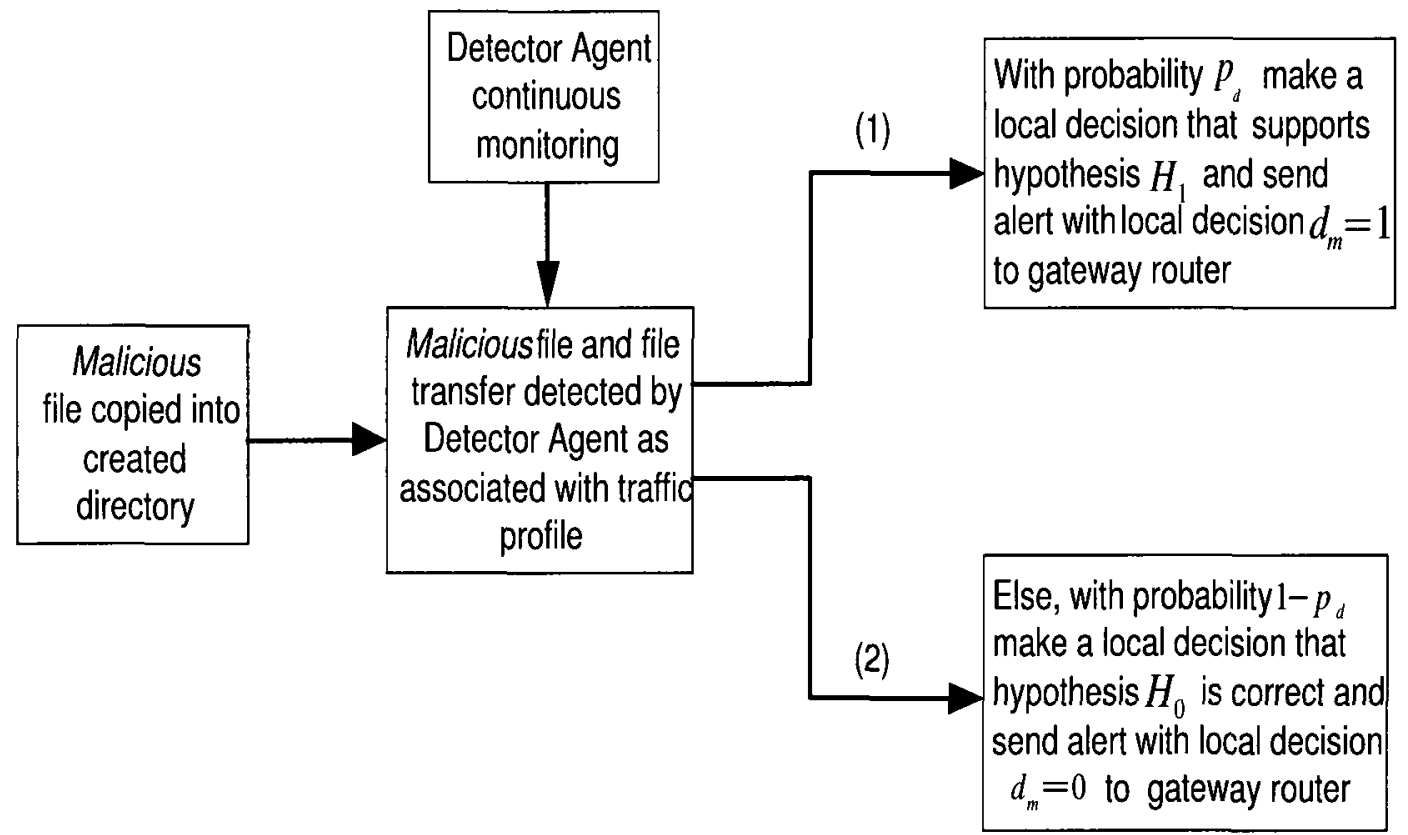

Figure 5.2: Implementation of host detection of malicious intrusion on the $m^{\text {th }} \mathrm{DE}$.

note the false detection probability of the $q^{t h}$ individual detector. Both $p_{d q}$ and $p_{f q}$ depend on the quality of the detector. In our implementation, the DEs are homogeneous $\ddagger$ since all DEs are assumed to run the same anomaly host-based intrusion detection software, hence $p_{d q}=p_{d}$ and $p_{f q}=p_{f}$, for all $q$. Also, $p_{d}>p_{f}$. See Table 5.1 for the relationship between $H_{1}, H_{0}$ and $p_{d}, p_{f}$ for each individual detector. Fig. 5.2 and Fig. 5.3 show block diagrams of our implementation of host detection and alerting.

Table 5.1: Relationship between $H_{1}, H_{0}$ and $p_{d}, p_{f}$ for individual detectors

\begin{tabular}{ccr}
\hline \hline True Nature & \multicolumn{2}{l}{ Local detector decision } \\
\hline & $H_{1}$ & $H_{0}$ \\
$H_{1}$ & $p_{d}$ & $1-p_{d}$ \\
$H_{0}$ & $p_{f}$ & $1-p_{f}$ \\
\hline
\end{tabular}

For a particular profile $i$, let $m_{i}$ be the total number of detectors in the target cell with observations of profile $i$ and $u_{i}$ be the total number of such detectors with individual local decisions which favor $H_{1}$. If we assume the observations on individual DEs are conditionally $\ddagger$ The implementation can be modified to use heterogeneous DEs with different $p_{d}$ and $p_{f}$ values. 


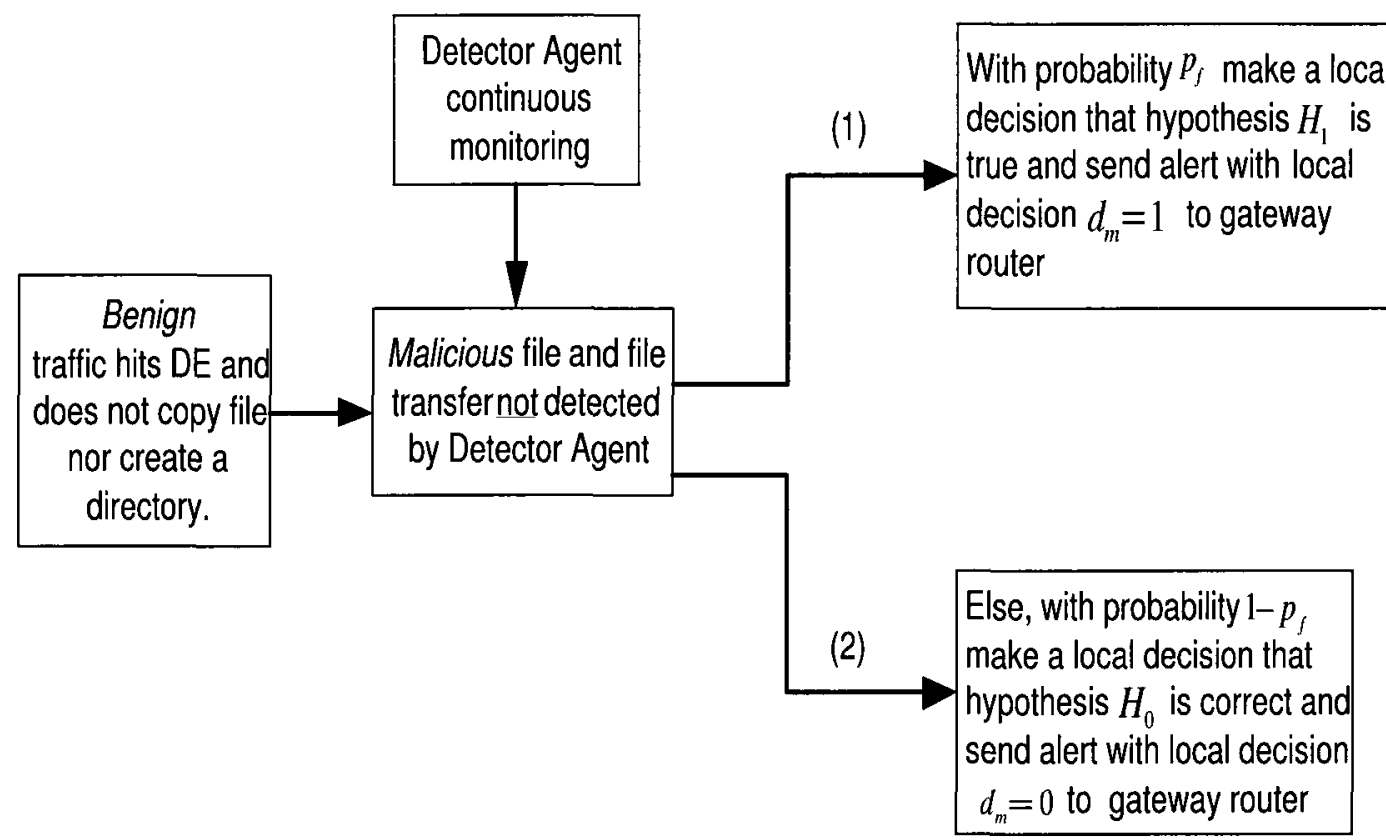

Figure 5.3: Implementation of host detection of benign intrusion on the $m^{\text {th }}$ DE.

Table 5.2: Parameters for GEP-based correlation algorithm

\begin{tabular}{|l|l|}
\hline Notation & Explanation \\
\hline$P_{D i}$ & Combined probability of positive detection for traffic profile $i$ \\
\hline$p_{d q}$ & Detection probability for the $q^{t h}$ individual detector \\
\hline$p_{f q}$ & False detection probability for the $q^{t h}$ individual detector \\
\hline$d_{q}^{i}$ & Individual local binary decision by the $q^{t h}$ DE on intrusion attempts due to profile $i$ \\
\hline$\underline{d}^{2}$ & Vector of local host decisions concerning profile $i$ \\
\hline$\Lambda\left(\underline{d}^{2}\right)$ & GEP likelihood ratio for optimized fused decision concerning profile $i$ \\
\hline$\gamma$ & GEP likelihood ratio threshold, also equivalent to $\frac{C_{10}}{C_{01}}$ \\
\hline$C_{a b}$ & Cost or penalty associated with a detector decision $a$ when the true hypothesis is $H_{b}$ \\
\hline$m_{i}$ & Total number of detectors with observations of profile $i$ \\
\hline$u_{i}$ & Total number of detectors with observations of profile $i$ and that favor $H_{1}$ \\
\hline$v_{i}$ & Number of detectors which favor $H_{1}$ required to minimally satisfy $\Lambda\left(\underline{d}^{2}\right) \geq \gamma$ \\
\hline
\end{tabular}

independent given hypotheses $H_{1}$ and $H_{0}$, then according to GEP, the conditional probabilities at the gateway router are:

$$
\begin{aligned}
& P\left(\underline{d}^{i} \mid H_{1}\right)=p_{d}^{u_{i}} *\left(1-p_{d}\right)^{m_{i}-u_{i}} \\
& P\left(\underline{d}^{i} \mid H_{0}\right)=p_{f}^{u_{i}} *\left(1-p_{f}\right)^{m_{i}-u_{i}}
\end{aligned}
$$


Hence, the likelihood ratio test in (5.1) is equivalent to,

$$
\Lambda\left(\underline{d}^{i}\right)=\frac{P\left(\underline{d}^{i} \mid H_{1}\right)}{P\left(\underline{d}^{i} \mid H_{0}\right)}=\left(\frac{p_{d}}{p_{f}}\right)^{u_{i}} *\left(\frac{1-p_{d}}{1-p_{f}}\right)^{m_{i}-u_{i}} \underset{\mathbf{D}=0}{\stackrel{\mathbf{D}=1}{\gtrless}} \frac{C_{10}}{C_{01}}=\gamma
$$

Based on computation of $\Lambda\left(\underline{d}^{i}\right)$, the likelihood ratio test in (5.2) determines whether the correlation algorithm considers traffic profile $i$ as a malicious traffic profile (i.e. $\mathbf{D}=1$ ) or a benign traffic profile (i.e. $\mathbf{D}=0$ ).

\subsubsection{Algorithm for handling multiple malicious attacks}

When multiple simultaneous malicious fast worm attacks occur and are detected using the correlation algorithm, there is a need to determine how to respond to the multiple attacks. Our proposed approach involves computing the combined probability of detection, $P_{D i}$ for each profile $i$ determined to be malicious using the likelihood ratio test in (5.2). $P_{D i}$ for each detected traffic profile $i$ is computed using the following expressions (see Table 5.2 for description of notations):

$$
P_{D i}=P\left(\Lambda\left(\underline{d}^{i}\right) \geq \gamma \mid H_{1}\right)
$$

For given $p_{f}$ and $p_{d}$ values on the host detectors, if we let $v_{i}$ be the minimum number of detectors which favor $H_{1}$ required to satisfy the condition $\Lambda\left(\underline{d}^{i}\right) \geq \gamma$ (determined using 5.2), then $P_{D i}$ defined in (5.3) can be expressed as:

$$
P_{D i}=\sum_{u_{i}: u_{i} \geq v_{i}}\left(\begin{array}{c}
m_{i} \\
u_{i}
\end{array}\right)\left(p_{d}\right)^{u_{i}}\left(1-p_{d}\right)^{m_{i}-u_{i}}
$$

Containment action for the multiple malicious traffic profiles is triggered in a sequence ordered by the value of $P_{D i}$ for the different profiles. Containment for profile $u$ is triggered before that of profile $v$ if,

$$
P_{D u}>P_{D v}
$$




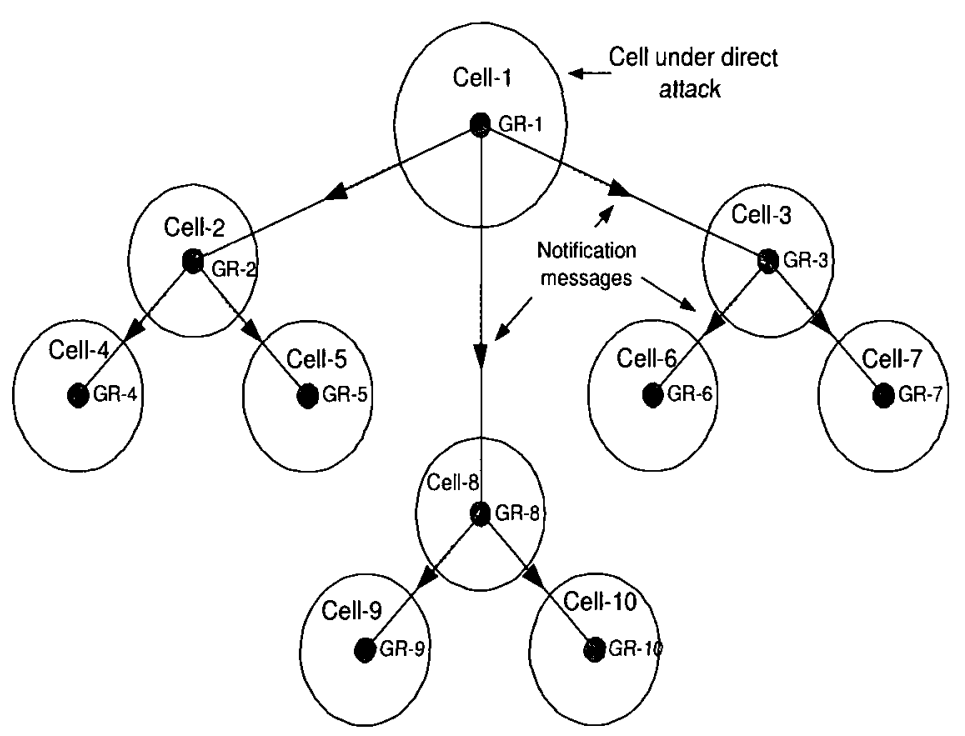

Figure 5.4: Distributed collaborative containment strategy

\subsubsection{Containment Technique}

The EDANC containment technique is based on a distributed defense scheme which uses a reactive blocking protocol (explained later in better detail) running on gateway routers to achieve collaborative containment. Using the detection and correlation technique explained above, the gateway router attached to the target cell under malicious attack identifies the malicious traffic profile(s) and triggers a containment action. First, the gateway router attached to the target cell (in Fig. 5.4, Cell-1 is the target cell and GR-1 is the attached gateway router) executes a reactive block ${ }^{\S}$ against the traffic profile(s) identified as malicious by automatically applying router filters against ingress connections that match the profile(s). The gateway router of the target cell (GR-1 in Fig. 5.4) then originates a block notification message to its neighbor routers (GR-2, GR-3 and GR-8 in Fig. 5.4) which informs them of the blocked profile(s). With the EDANC containment technique, all gateway routers run the reactive blocking protocol when a block notification message is received. The reactive blocking protocol that runs on the gateway routers determine how the routers respond to

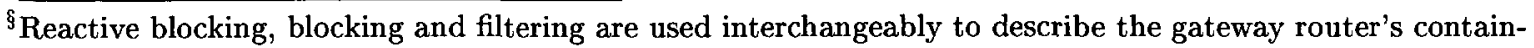
ment action. 
a block notification message. We describe the reactive blocking protocol below using the following processes.

\subsubsection{Filtering process}

The reactive blocking protocol starts by creating a router filter on the neighbor gateway router that receives the block notification message (in this case GR-2, GR-3 and GR-8 in Fig. 5.4 are the neighbor gateway routers of GR-1). The filter blocks ingress traffic that match the profile(s) contained in the block notification message from entering all cells or subnets existing on the neighbor gateway router.

\subsubsection{Monitoring process}

With the filter still applied, the neighbor router carries out real-time monitoring and recording of the number of hits on the filter caused by the blocked profile(s) for a time interval equivalent to $x$ seconds to verify actual existence of the suspected malicious worm activity. In our experiments, a tractable value was chosen for parameter $x$ based on experience. After $x$ seconds the algorithm computes a probing rate, $R_{i}$ for each blocked profile $i$ :

$$
R_{i}=\frac{\text { number of hits on filter by profile } i}{x}
$$

It then carries out the following conditional loop with chosen parameter $w$. Similar to $x$, a tractable value was chosen for parameter $w$ based on experience with our experiments. 


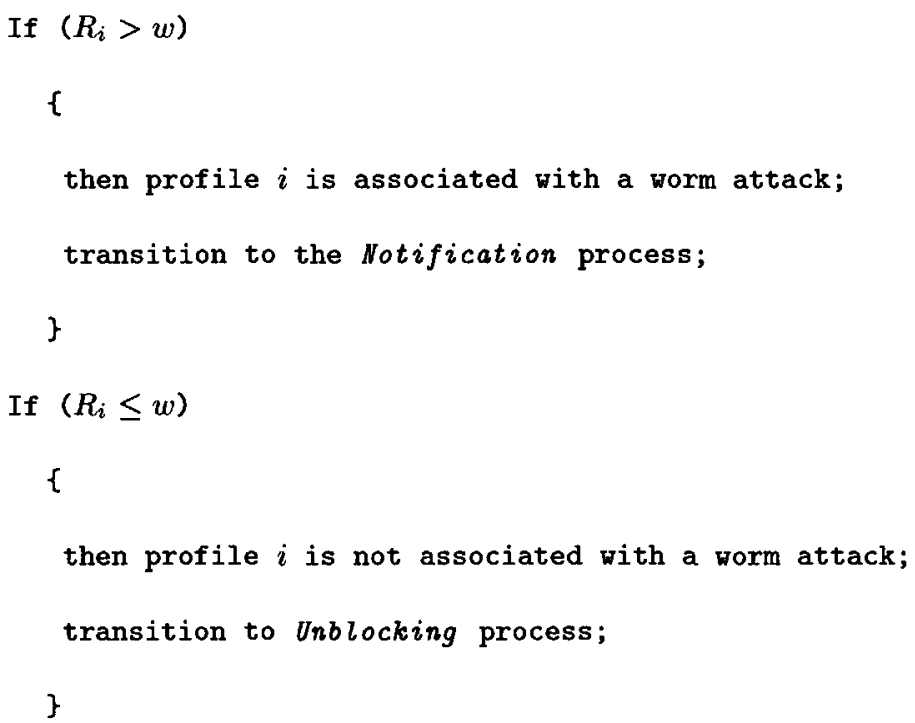

\subsubsection{Notification process}

During this process, the neighbor router notifies its own neighbor routers of the suspicious profile by sending a block notification message to them. This also triggers the reactive blocking protocol on the new neighbor gateway routers. Note that a block notification message is not sent back the path it was earlier received on. This ensures that a gateway router that originates a block notification message does not receive the same message from its neighbors. As an example, in Fig. 5.4, GR-2 which received an initial block notification message from GR-1 will send a block notification message to GR-4 and GR-5 but will not send the message back to GR-1.

\subsubsection{Unblocking process}

In this state the filter is removed from the neighbor router to prevent a denial of service on non-malicious traffic. 
Fast spreading malicious worms are known to exhibit probing rates in the order of tens of thousands probes/second. In the event of a fast spreading worm attack, the reactive blocking protocol on most gateway routers will get to run the Notification process if $x$ and $w$ parameters are properly chosen. In that scenario, all enterprise routers quickly become aware of the suspicious profile(s) and establish filters against ingress traffic that match the profile(s) thus achieving enterprise-wide fast and automated containment.

\subsection{Detection Interval Analysis}

For a particular malicious worm traffic with profile $i$, detection interval, $t_{D, i}$ is the interval between the time the worm scan first hits a target cell and the time the worm is positively detected in the target cell. As explained in Section 5.2.2, for given $p_{f}$ and $p_{d}$ values on the host detectors, a minimum of $v_{i}$ DEs in the target cell must make a positive detection of the malicious intrusion to satisfy the condition $\Lambda\left(\mathbf{x}_{\mathbf{i}}\right) \geq \gamma$ for the GEP optimized fused decision $\mathbf{D}=1$ to be made. Hence, the detection interval, $t_{D, i}$ is the interval between the time the worm scan first hits a target cell and the time a minimum of $v_{i}$ DEs in the target cell make a positive detection of the malicious intrusion. The detection interval, $t_{D, i}$ comprises the total inter-infection interval, $t_{V, i}$ and the total time to infect, $t_{i n f e c t, i}$ for the particular malicious traffic with profile $i$ (i.e. $t_{D, i}=t_{V, i}+t_{\text {infect }, i}$ ). These parameters will be explained in the following sections. We also show in Section 5.3.2 that since $t_{\text {infect }, i}$ is negligible for fast scanning worms, $t_{D, i} \approx t_{V, i}$. 


\subsubsection{Total inter-infection interval, $t_{V, i}$}

Inter-infection interval is the time interval between successive hits experienced by hosts in a target cell as a result of worm scans. The total inter-infection interval, $t_{V, i}$ is the sum of time intervals between successive worm scans of the DEs in the target cell until a minimum of $v_{i}$ DEs in the target cell make a positive detection of intrusion.

We model scanning of hosts in the target cell by a Poisson process with an average rate of $r$ hosts/second $(\mathrm{h} / \mathrm{s})$. Use of the Poisson distribution to model random scanning behavior of malicious worms is not uncommon. Authors in [55] [35] used the Poisson process to model the behavior of random scanning worms. Using the Poisson model, the inter-infection interval between hosts is therefore an exponential random variable with mean $\frac{1}{r}$ and the total interinfection interval, $t_{V, i}$ is the sum of inter-infection intervals until at least $v_{i}$ DEs in the target cell make a positive detection. Two network scenarios are possible:

1. The total number of hosts in the target cell is $W$ and the number of detectors (DEs) in the target cell is $m$, where $m<W$.

2. All hosts on the target cell function as detectors, hence $m=W$.

\subsubsection{Scenario 1: $m<W$}

In this scenario, we assume that there are a total of $W$ hosts in the target cell comprising $m$ DEs and therefore $W-m$ non-detectors (non-DEs) ๆ, and that $k_{i}$ hosts (comprising DEs and non-DEs) in the target cell are scanned by the intrusion traffic with profile $i$ before the condition for detection is met (i.e $\Lambda\left(\mathbf{x}_{\mathbf{i}}\right) \geq \gamma$ ). Then, the minimum total inter-infection $\mathrm{I}_{m \geq v_{i}}$ 
interval, $t_{V \min , i} \|$ will be an Erlang- $k_{i}$ random variable with mean $\frac{k_{i}}{r}$. But,

$$
k_{i}=v_{i}+z_{i} \quad v_{i} \leq m
$$

where $z_{i}$ is the number of non-DEs that were scanned before a positive detection decision is made. $z_{i}$ is a random variable with values that range between 0 and $W-m$. Since $z_{i}$ can have values between 0 and $W-m$ with equal probability, we can assume $z_{i}$ follows a uniform distribution, $z_{i} \sim U(0, W-m)$. Hence,

$$
E\left[t_{V \min , i}\right]=E\left[\frac{k_{i}}{r}\right]=E\left[\frac{v_{i}+z_{i}}{r}\right]=\frac{2 v_{i}+W-m}{2 r} \quad v_{i} \leq m
$$

\subsubsection{Scenario 2: $m=W$}

In this scenario, we assume that the number of detectors, $m$ in the target cell is equivalent to the total number of hosts $W$ in the target cell and that $k_{i}$ hosts (comprising only DEs) in the target cell are scanned by the intrusion traffic with profile $i$ before the condition for detection is met. Then $k_{i}=v_{i}$. The minimum total inter-infection interval, $t_{V m i n, i}$ is also an Erlang- $v_{i}$ random variable with mean $\frac{v_{i}}{r}$.

Since both $v_{i}$ and $r$ are constants,

$$
E\left[t_{V \min , i}\right]=E\left[\frac{v_{i}}{r}\right]=\frac{v_{i}}{r}
$$

$t_{V \min , i}$ is the minimum total inter-infection interval which occurs when hosts in the target cell are scanned only once in a single worm attack instance. Multiple host scans by a particular malicious worm will result in greater total inter-infection interval for the worm. 


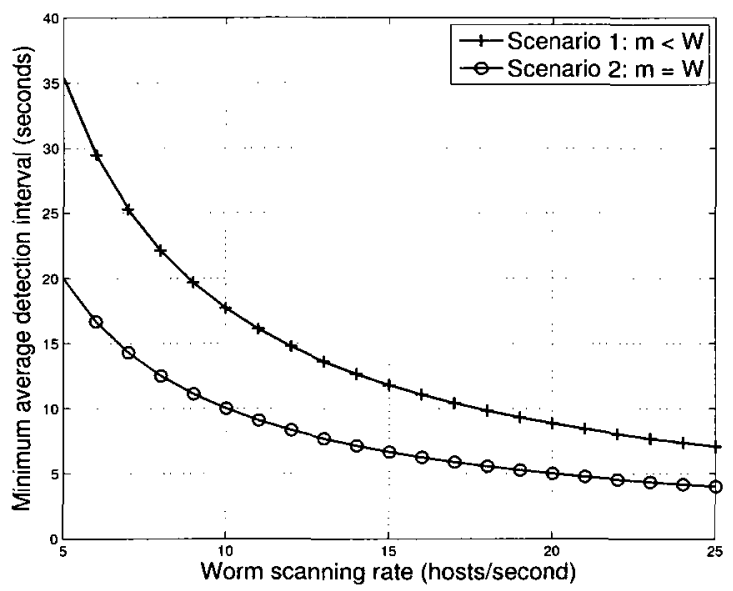

Figure 5.5: Average detection interval using (5.5), (5.6) and (5.7). $W=254, v_{i}=20$.

\subsubsection{Total time to infect, $t_{\text {infect }, i}$}

This is the time interval it takes to scan and infect a vulnerable host in the target cell. This time is largely dependent on the nature of the intrusion attack and the vulnerability being exploited on the endpoint. For analysis, we assume that $t_{\text {infect, } i}$ is negligible for fast spreading worms. Therefore,

$$
\bar{t}_{D \min , i} \approx E\left[t_{V \min , i}\right]
$$

where $\bar{t}_{D m i n, i}$ is the minimum average detection interval for profile $i$.

Using simulations, Fig. 5.5 was generated using (5.5), (5.6) and (5.7). Fig. 5.5 shows that using our technique $\bar{t}_{D m i n, i}$ decreases progressively with increase in worm scanning rate, and intrusion attacks of over $15 \mathrm{~h} / \mathrm{s}$ are detectable within 13 seconds after starting the attack. Detection interval is even less (under 8 seconds) if $m=W$. 


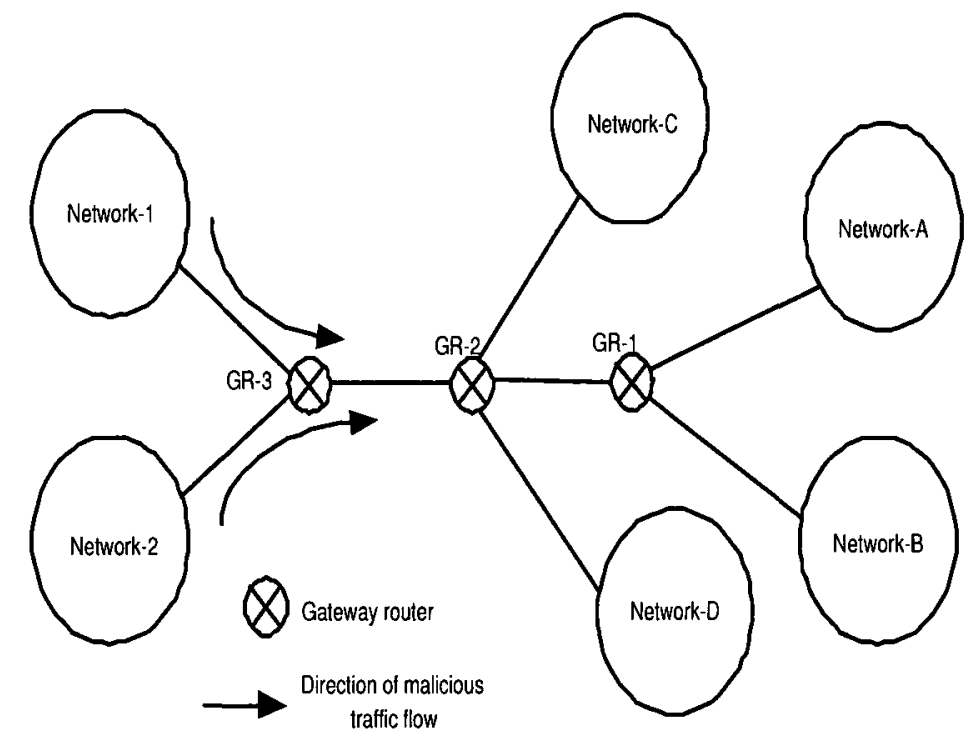

Figure 5.6: Test-bed used for experimentation

\subsection{Experimentation}

\subsubsection{Description of test-bed setup}

Fig. 5.6 shows the topology of our live testbed which will be described in more detail in this section. Worm attacks are sourced from Network-1 and Network-2 and targeted at vulnerable hosts in Network-A, Network-B, Network-C and Network-D. Detector endpoints (DEs) that run the EDANC detection algorithm are located within the target cells and communicate with their gateway router (GR-1 and GR-2). The gateway router runs our GEP-based correlation algorithm.

To evaluate the functionality and performance of the EDANC detection technique on a live testbed, we emulated scanning worm attacks using a modified blaster worm source code [52]. To emulate multiple malicious attacks, the source code was used to instrument two worms that performed two different actions on the target hosts. The first, worm-1 was instrumented to create a directory named/root/infected-1 on the target host and copy a file named 
malicious-1 into that directory over TCP port 888 . The second, worm- 2 was instrumented to create a directory named/root/infected-2 on the target host and copy a file named malicious-2 into that directory over UDP port 999. Hosts in Network-1 and Network-2 were used to launch worm-1 and worm-2 attacks respectively on hosts in the target networks (Network A, Network B, Network C, and Network D). We used OpenVZ virtualization ** [69] to create the required vulnerable host population in the target networks. Upto 128 virtual hosts per workstation were created on a number of Linux workstations running OpenVZ kernel-2.6.22 to emulate a class $\mathrm{C}$ network population in each target network. Scanning rates of upto 70 hosts per second $(\mathrm{h} / \mathrm{s})$ were generated from a single worm-1 or worm-2 attack instance. In comparison, the Code Red worm [49] infected 359000 hosts in less than 14 hours, equivalent to an average infection rate in the order of $7.1 \mathrm{~h} / \mathrm{s}$. The Witty worm [62] infected 110 hosts in the first 10 seconds, equivalent to an average infection rate of about $11 \mathrm{~h} / \mathrm{s}$ while the Slammer worm [48] infected more than 75,000 hosts within 10 minutes, equivalent to an average infection rate of over $125 \mathrm{~h} / \mathrm{s}$.

For our test, host-based anomaly detection on the DEs was emulated using a detector agent (DA) ${ }^{\dagger \dagger}$ that constantly monitored the directory structure and directory content on the DE as well as the file transfer logs on the DE. The detector agent generates an alert when a file named malicious-1 or malicious- 2 is transfered into a directory named/root/infected-1 or /root/infected-2 respectively on the DE. In our implementation, a snort-based IDS was used for real-time recording of incoming packets on the DE in combination with a detector agent created using Linux scripts $\ddagger$. Fig. 5.2 and Fig. 5.3 in Section 5.2.2.1 show our implementation of host detection and alerting. As explained in Section 5.2.2.1, a $\gamma=\frac{C_{10}}{C_{01}}$ value of 2.0 was used in our experiments. All hosts on the target networks functioned as detectors, hence $m=W$ (using notations explained in Section 5.3.1). The fast worm

\footnotetext{
** OpenVZ is an operating system-level virtualization technology based on the Linux kernel and operating system.

${ }^{\dagger}$ Combination of Linux scripts and Snort-based IDS.

${ }^{\ddagger}$ Note that our emulation of host-based detection with detector agents and snort-based real-time logging was only used to demonstrate the EDANC scheme. Other host Anomaly Intrusion Detection System (AIDS) software such as Thirdbrigade's host AIDS, Cisco's Security Agent and Tripwire's host AIDS can be used for host-based detection in enterprise deployment of the EDANC scheme.
} 
detection window parameter, $t_{f}$ on the detectors was set to 20 seconds.

The purpose of the experiments was to demonstrate how the EDANC detection technique can be used for detecting fast propagating worm attacks. It may not be representative of all the possible fast worm attack scenarios that exist or may exist on the Internet today. Note that the focus of this work is to develop and demonstrate a new technique for combining local detector decisions from multiple host detectors with known $p_{d}$ and $p_{f}$ values for improved detection, and not to demonstrate the detection capabilities of any particular host detection software or tool.

For data generation and analysis of results we are interested in, each experiment was run 30 times and the average value as well as a confidence interval based on a $95 \%$ confidence level was computed.

\subsubsection{Experiment 1: EDANC detection interval}

In this experiment, a single attacking host in Network-1 was used to launch direct attacks on hosts in the target networks. The objective of the experiment was to evaluate the responsiveness of the EDANC scheme in detecting unauthorized malicious intrusions in a target network. The worm scanning rate was varied to investigate its impact on the EDANC detection scheme. The probability of detection, $p_{d}$ and the probability of false detection $p_{f}$ of the individual detectors were set to 0.99 and 0.01 respectively. Similar values were used in $[36]$.

Fig. 5.7 shows that average detection interval observed on GR-1 and GR-2 reduces progressively with increase in worm scanning rate. These observations concurs with results obtained analytically in Section 5.3 (see Fig. 5.5). Fig. 5.7 shows that the EDANC scheme is capable of automatically detecting malicious intrusion attacks with scanning rate of over $15 \mathrm{~h} / \mathrm{s}$ 


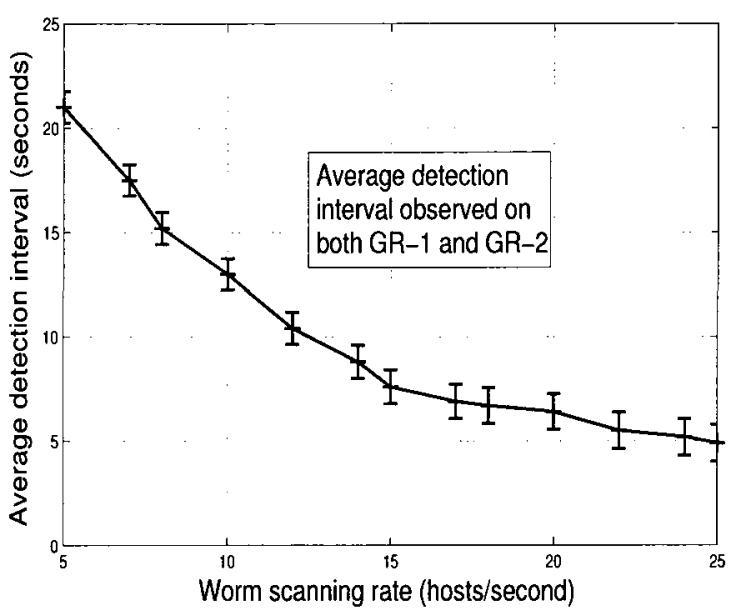

Figure 5.7: Experiment 1: Average detection interval. $W=254, \gamma=2.0, p_{d}=0.99, p_{f}=0.01$.

within an average of 8 seconds (with $m=W$ ) after starting the attack on a network with cells containing 254 hosts. In comparison, simulation results in [50] suggest that an effective worm containment should require a reaction time of at least 60 seconds. The responsiveness of the EDANC intrusion detection scheme is therefore reasonable.

\subsubsection{Experiment 2: Effect of varying probability of detection, $p_{d}$ on EDANC detection}

We performed this experiment to investigate the impact of varying the probability of detection $p_{d}$ of the individual detectors on the GEP-based correlation algorithm and fused decision explained in Section 5.2.2.1. A single attacking host in Network-1 was used to launch direct attacks on hosts in the target networks at a scanning rate of $20 \mathrm{~h} / \mathrm{s}$ and the attack session was stopped when a detection decision was made by the correlation algorithm running on GR-1 and GR-2. The probability of false detection, $p_{f}$ of the individual detectors was set to 0.01 . The number of hosts in a target cell was set to 254 . Several sets of experiment runs were made, and in each set the value of the probability of detection $p_{d}$ of the individual detectors in the target network was modified. Fig. 5.8 shows that a reduction 


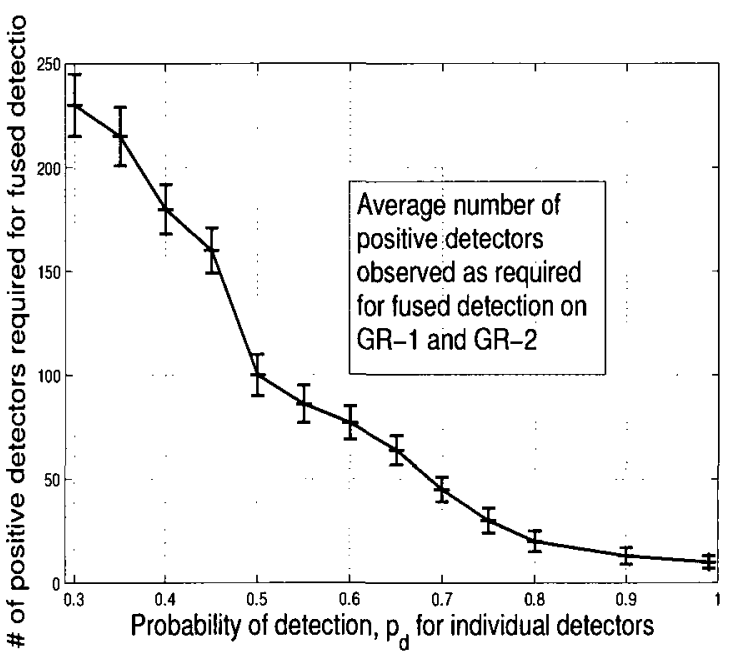

Figure 5.8: Experiment 2: Impact of varying, $p_{d}$ on EDANC detection. $W=254, p_{f}=0.01$. Worm scanning rate $=20 \mathrm{~h} / \mathrm{s} . \gamma=2.0$.

in $p_{d}$ results in a corresponding increase in the number of positive detectors required to meet the conditions for a fused decision $\mathbf{D}=1$ (i.e. $v_{i}$ ) using the GEP likelihood ratio test in (5.2). Positive detectors refer to detectors which favor the $H_{1}$ hypothesis. This result is somewhat intuitive since a reduction in $p_{d}$ means that the individual detectors are less accurate in detecting malicious traffic (see Table 5.1). In this scenario, the correlation algorithm therefore required more reports of individual detector local decisions to arrive at a fused decision. Conversely, more accurate detectors (with high $p_{d}$ values) will cause the correlation algorithm to require less number of individual detector local decisions to arrive at a fused decision.

\subsubsection{Experiment 3: Effect of varying probability of false detection, $p_{f}$ on EDANC detection}

This experiment was carried out to investigate the impact of varying the probability of false detection $p_{f}$ of the individual detectors on the GEP-based correlation algorithm and fused decision. Two attacking hosts, one in Network-1 and the other in Network-2 were used to launch direct malicious attacks on hosts in the target networks at a scanning rate of $20 \mathrm{~h} / \mathrm{s}$ 


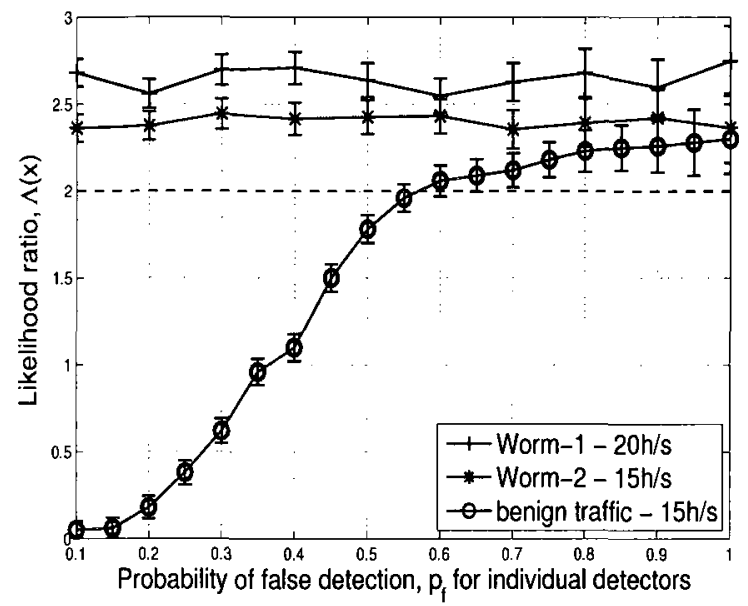

Figure 5.9: Experiment 3: Impact of varying, $p_{f}$ on EDANC detection. $W=254$. Worm scanning rate $=20 \mathrm{~h} / \mathrm{s}, \gamma=2.0, p_{d}=0.99$.

and $15 \mathrm{~h} / \mathrm{s}$ respectively. The attack sessions were stopped when a detection decision was made by the correlation algorithm running on GR-1 and GR-2. A third host in Network-1 was used to scan the target networks with benign background traffic at a rate of $15 \mathrm{~h} / \mathrm{s}$. The probability of detection, $p_{d}$ of the individual detectors was set to 0.99 . Several sets of experiment runs were made, and in each set the value of the probability of false detection $p_{f}$ of the individual detectors in the target network was modified. Fig. 5.9 shows that changes in the $p_{f}$ parameter for individual detectors does not have significant impact on the detection of malicious traffic if the $p_{d}$ parameter for the detectors is fixed. This is expected since detection of malicious traffic is dependent on $p_{d}$ but has no dependency on $p_{f}$ (see Table 5.1). For the benign traffic, Fig. 5.9 shows that an increase in $p_{f}$ results in a corresponding increase in the likelihood that the benign background traffic will be falsely detected as malicious. This is because as $p_{f}$ increases the number of detectors that wrongly favor the $H_{1}$ hypothesis for benign traffic hits increases progressively. Fig. 5.9 shows that for $p_{f} \geq 0.55$ our GEP algorithm exhibited false detection of benign traffic. 


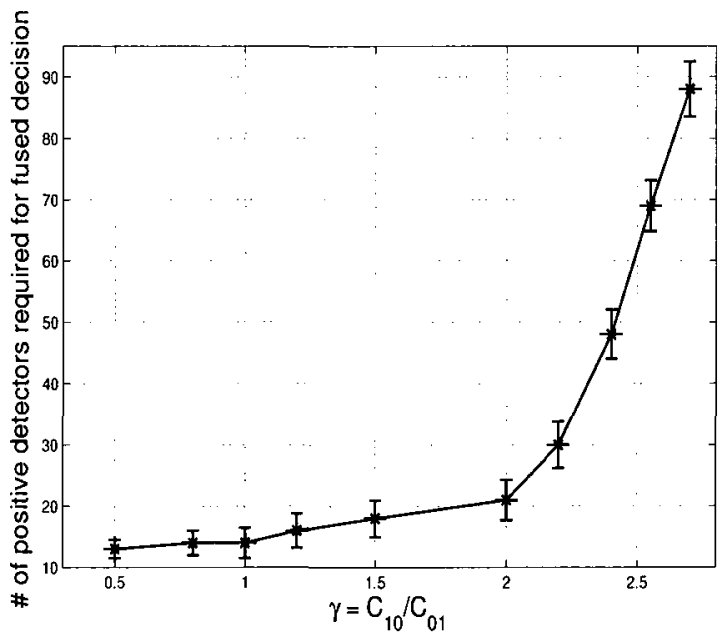

Figure 5.10: Experiment 4: Impact of $\gamma=\frac{C_{10}}{C_{01}}$ on EDANC detection. $W=254, p_{d}=0.99$, $p_{f}=0.01$.

\subsubsection{Experiment 4: Effect of varying $\gamma=\frac{C_{10}}{C_{01}}$ on EDANC detection}

In this experiment, a single attacking host in Network-1 was used to launch direct attacks on hosts in the target networks and the attack session was stopped when a detection decision was made by the correlation algorithm running on GR-1 and GR-2. The probability of detection, $p_{d}$ and the probability of false detection $p_{f}$ of the individual detectors were set to 0.99 and 0.01 respectively. Similar values were used in [36]. The number of hosts in a target cell was set to 254. Several sets of experiment runs were made, and in each set the value of $\gamma=\frac{C_{10}}{C_{01}}$ was modified. Figure 5.10 shows that an increase in $\gamma$ results in a corresponding increase in the average number of positive detectors required to meet the conditions for a fused decision $\mathbf{D}=1$ (i.e. $v_{i}$ ) using the likelihood ratio test in (5.2). With $\gamma=2.0$, we observed that the number of positive detectors required to meet the conditions for a fused decision $\mathbf{D}=1$ remained an average of 20 . In practical deployments with homogeneous detectors, security engineers can choose a desirable number of positive detectors required for a fused decision and tune $v_{i}$ to match this chosen number by adjusting $\gamma$. 


\subsubsection{Experiment 5: Comparison: Accuracy of EDANC GEP-based de- tection versus single detector detection}

The objective of this experiment was to compare the accuracy of EDANC GEP-based detection and single host detector detection using receiver operating characteristic (ROC) curves. On our test-bed (Fig. 5.6), only attacking hosts from Network-1 and host detectors in Network-A were used in this experiment.

\subsubsection{Generating ROC curve for EDANC GEP-based detection}

The host detectors in Network-A (254 hosts) and gateway router GR-1 were configured to run the GEP algorithm. Two sets of experiments were performed. In the first set, one malicious host from Network-1 was used to scan hosts in Network-A with scanning rate $20 \mathrm{~h} / \mathrm{s}$. The $p_{f}$ and $p_{d}$ parameters for the detectors in Network-A were varied and for each $\left(p_{f}, p_{d}\right)$ pair 30 runs of the scan were carried out and fused decisions on the gateway router were observed. To generate data for the ROC curve for GEP-based detection, we computed the detection rate (i.e. true positive rate) using (5.8) for each $\left(p_{f}, p_{d}\right)$ pair used in the experiment.

Detection rate $=$ True positive rate $=\frac{\text { Number of malicious runs with fused decision, } \mathbf{D}=1}{\text { Total number of runs with malicious traffic }}$

In the second set of experiments, one benign host from Network-1 was used to scan hosts in Network-A with scanning rate $20 \mathrm{~h} / \mathrm{s}$. The $p_{f}$ and $p_{d}$ parameters for the detectors in the target networks were varied using same values as in the first set of experiments. For each $\left(p_{f}, p_{d}\right)$ pair several runs of the scan were carried out and fused decisions on the gateway router were observed. We computed the false positive rate using (5.9) with each $\left(p_{f}, p_{d}\right)$ 
pair.

False positive rate $=\frac{\text { Number of benign runs with fused decision, } \mathbf{D}=1}{\text { Total number of runs with benign traffic }}$

Using the computed detection rates and false positive rates, the ROC curve for GEP-based detection in Fig. 5.11 was generated. Fig. 5.11 also shows the $\left(p_{f}, p_{d}\right)$ values used in each of the experiment runs.

\subsubsection{Generating ROC curve for single host detector detection}

Using only a single host detector in Network-A, the first and second set of experiments as described above were performed and the host detector local decisions were observed. Using (5.8) and (5.9), the detection rates (i.e. true positive rates) and false positive rates were computed and used to generated the ROC curve in Fig. 5.11 for a single host detector decision.

Fig. 5.11 shows the ROC curves for both GEP-based detection and the single host-based detection. The ROC curves show that the EDANC GEP-based detection technique results in a much more accurate intrusion detection system than the use of a single host-based intrusion detector.

\subsubsection{Experiment 6: Multiple concurrent worm attacks}

In this experiment, single attacking hosts from different networks (Network-1 and Network-2 in Fig. 5.6) were used to launch different attacks (worm-1 and worm-2 respectively) on hosts in the target networks. The single attacking hosts from Network-1 and Network-2 scanned the target networks at a scanning rates of $20 \mathrm{~h} / \mathrm{s}$ and $15 \mathrm{~h} / \mathrm{s}$ respectively. Parameter $p_{d}=0.9$ and $p_{f}=0.01$ were configured on the host detectors. We observed that both worms were 


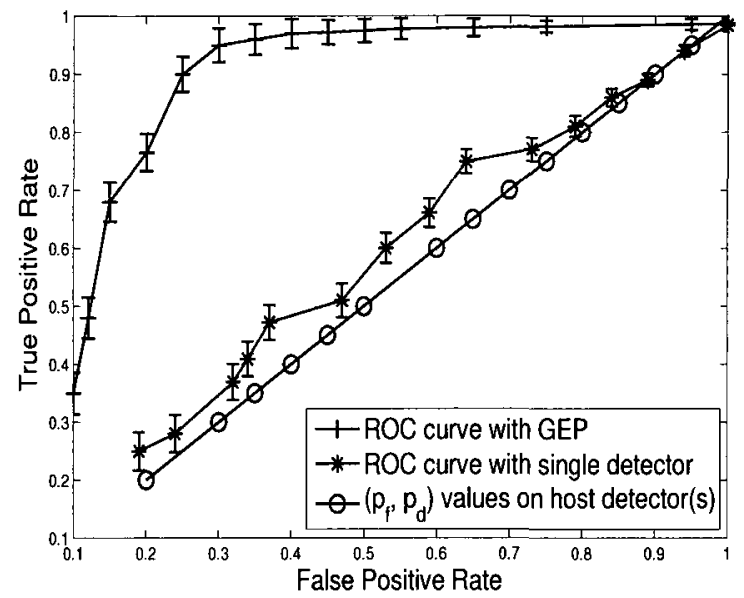

Figure 5.11: Experiment 5: ROC curves for EDANC GEP detection versus single host detection. $W=254 . \gamma=2.0$.

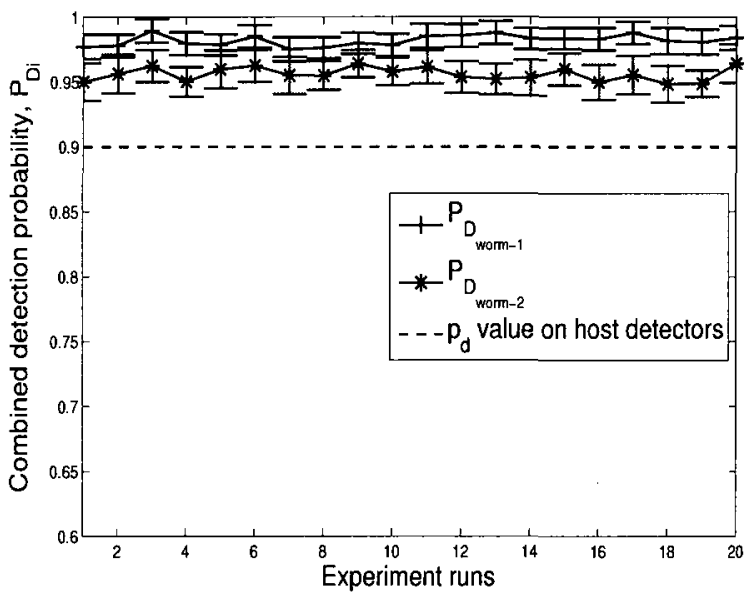

Figure 5.12: Experiment 6: Combined detection probability, $P_{D i}, W=254, \gamma=2.0, p_{d}=0.9$, $p_{f}=0.01$.

identified as malicious (i.e. fused decision $\mathbf{D}=1$ ) using the GEP likelihood ratio test in (5.2). The combined detection probability for worm-1 and worm-2 traffic profiles are captured in Fig. 5.12. We observed that though both worms were identified as malicious and selected for containment, worm-1 (the faster worm) was blocked first since the combined probability of detection (Fig. 5.12) for worm-1 was greater than that of worm-2 (i.e. $P_{D_{\text {worm-1 }}}>P_{D_{\text {worm-2 }} \text { ) }}$ as explained in (5.4). 


\subsection{Chapter Summary}

In this chapter, we developed the Endpoint Detection And Network Containment (EDANC) scheme for distributed detection and collaborative defense against fast spreading worms. The EDANC detection and correlation engine is based on the Generalized Evidence Processing theory, a decision level multi-sensor data fusion technique. With GEP theory, the evidence collected by the distributed detectors determines the probability associated with a decision under a hypothesis. The probability mass assignment associated with each host detector decision is combined to arrive at a improved fused decision by minimizing a decision risk function. GEP theory is known to have advantages over the two major evidence combining theories that have dominated the field of distributed evidence processing - the Bayesian theory and the Dempster-Shafer theory. As far as our literature survey revealed, and to the best of our knowledge we were the first in [4] to use the GEP theory as a theoretical foundation for intrusion detection of malicious traffic in computer networks.

We presented an analysis of EDANC detection interval for a fast spreading scanning worm and experimented with the EDANC scheme on a live test-bed. Results obtained from experimentation concurred with analytical results. The results also validate the functionality of the EDANC detection technique for fast scanning malicious worms. Some useful deductions from the experimental results include the following:

1. The EDANC scheme is capable of automatically detecting malicious intrusion attacks with scanning rate of over $15 \mathrm{~h} / \mathrm{s}$ within an average of 8 seconds after starting the attack on a network with cells (network segments) containing 254 hosts.

2. A reduction in the probability of detection, $p_{d}$ of the individual detectors results in a corresponding increase in the number of positive detectors required to meet the conditions for a fused decision $\mathbf{D}=1$ (i.e. decision that observed intrusion is malicious). We used homogeneous host detectors with same $p_{d}$ and $p_{f}$ values. Though somewhat 
intuitive, the results showed that with poor detectors (with low $p_{d}$ values) the correlation algorithm required more reports of individual detector local decisions to arrive at a fused decision. Conversely, more accurate detectors (with higher $p_{d}$ values) caused the correlation algorithm to require less number of individual detector local decisions to arrive at a fused decision.

3. Changes in probability of false detection, $p_{f}$ values for individual detectors does not have significant impact on the detection of malicious traffic if the $p_{d}$ value for the detectors is fixed. This is expected since detection of malicious traffic is dependent on $p_{d}$ but has no dependency on $p_{f}$ (see Table 5.1). However, an increase in $p_{f}$ results in a corresponding increase in the likelihood that a benign traffic will be falsely detected as malicious. With $p_{f} \geq 0.55$ our GEP algorithm exhibited false detection of benign traffic.

4. An increase in $\gamma=\frac{C_{10}}{C_{01}}$ (i.e. ratio of the cost of a false positive decision to the cost of a false negative decision) results in a corresponding increase in the number of positive detectors required to meet the conditions for a fused decision $\mathbf{D}=1$.

5. Generated ROC curves show that the EDANC GEP-based detection technique exhibits much better accuracy than the use of a single host detector for detection.

6. In multiple concurrent malicious attack scenarios, even though all the malicious attack profiles are identified as malicious and selected for containment, the profiles are blocked (or contained) in a sequence ordered by the combined probability of detection $\left(P_{D_{i}}\right)$ computed for the profiles. In our experiments, we observed that the scanning rate of a worm had a direct relationship with $P_{D_{i}}$ computed for that worm profile. The worm with a faster scanning rate exhibited a greater $P_{D_{i}}$ and was blocked first. 


\section{Chapter 6}

\section{Modeling EDANC Collaborative Defense}

\subsection{Introduction}

Recent advancements in Internet fast worm propagation techniques has generated interest in the development of appropriate defense techniques against such worms. Modeling the behavior of worm defense techniques to better understand and measure their defense capabilities is crucial to developing effective defenses. This chapter presents a discrete-time model for the EDANC collaborative network containment technique which we refer to as the Analytical Active Worm Containment (AAWC) model. The EDANC collaborative containment technique was explained earlier in Section 5.2.3. The AAWC model captures the protection capability of the EDANC technique by modeling the host population protected from fast spreading, scanning intrusion attacks such as worms in a large scale network. Analysing the model alongside an existing well known discrete-time worm propagation model - the AAWP (Analytical Active Worm Propagation) model, we demonstrate quantitatively the effective- 
ness of the EDANC containment technique in defending against fast spreading scanning worms. Further, we investigate the introduction of immunization to the EDANC containment technique and show the resultant effect on a vulnerable population under attack using the developed model.

The main contributions in this chapter are:

$\dagger$ We develop the Analytical Active Worm Containment (AAWC) model, a discrete-time model used to model a vulnerable host population protected as a result of EDANC collaborative defense in a large scale network.

† Using the AAWC model we demonstrate quantitatively the collaborative containment capability of the EDANC scheme. We define containment of an infectious worm as the complete halting of further worm spread. Therefore, when a fast spreading worm is contained using the EDANC scheme, significant portions of the vulnerable uninfected population are protected from infection. We also evaluate introducing immunization to the EDANC collaborative defense.

\subsection{Modeling}

In this section, we develop the Analytical Active Worm Containment (AAWC) model which is used to model host population protected as a result of the EDANC collaborative defense scheme in a large hierarchical network ${ }^{*}$ as depicted in Fig. 6.1 and Fig. 6.2. In Fig. 6.1 and Fig. 6.2 the nodes represent network routers, the level $L$ nodes represent gateway routers which contain cells ${ }^{\dagger}$ while level 0 represents the network core routers. Routers upstream of level $L$ do not contain cells. When a gateway router implements a blocking filter against

\footnotetext{
* The Internet and most well-designed large enterprise networks generally follow a hierarchical architecture [7] [42].

${ }^{\dagger}$ End hosts are logically located within a cell and a single gateway router typically contains multiple cells.
} 


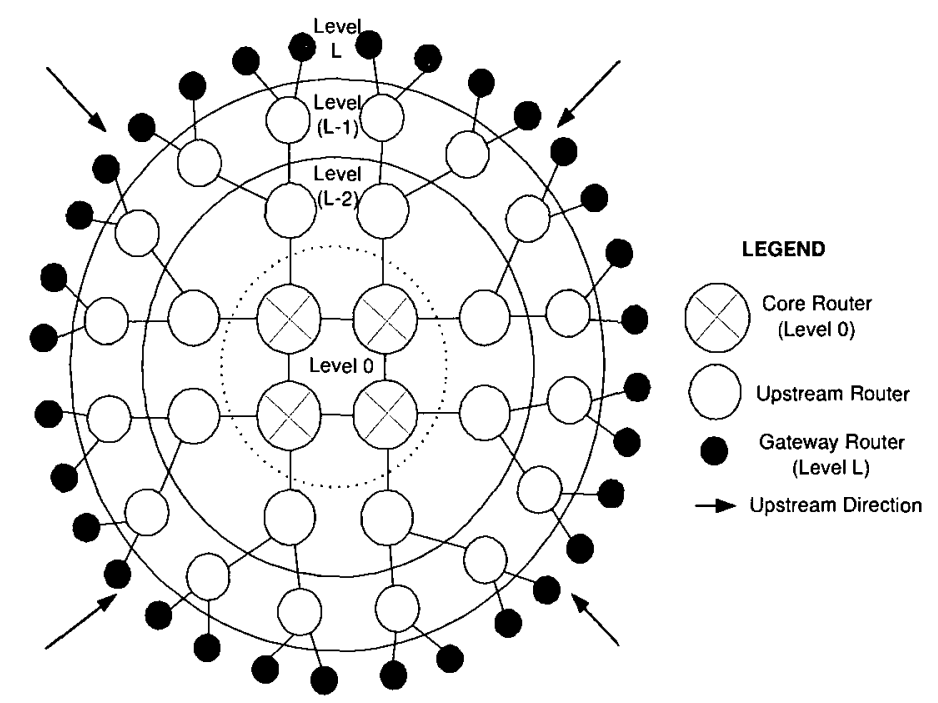

Figure 6.1: Typical large-scale hierarchical network topology.

an intrusion traffic, the filter is applied to all cells attached to the gateway router hence protecting them from the intrusion traffic. Also, when an upstream router implements a blocking filter against an intrusion traffic, all cells contained in gateway routers downstream of that upstream router are protected from the intrusion traffic. While we acknowledge that our hierarchical network topology is not representative of all real-life production networks, it depicts the general topology of a hierarchical network. Authors in [7] [42] emphasized that the Internet and most well-designed large enterprise networks generally follow a hierarchical architecture. We therefore considered our topology sufficient to demonstrate the protection capability of EDANC against large scale fast spreading worm epidemics.

Table 6.1: Parameters for network topology and AAWC model

\begin{tabular}{|l|l|}
\hline Notation & Explanation \\
\hline$L$ & number of hierarchical levels in network \\
\hline$y$ & number of nodes that connect to an upstream node \\
\hline$W$ & number of hosts in each cell \\
\hline$s$ & number of W-sized cells that exist on each GR \\
\hline$t_{r}$ & time intrusion traffic is released into the network \\
\hline$t(j)$ & time of containment at level $j$ in the hierarchical network \\
\hline$\delta$ & time interval for notification between routers \\
\hline$M(j)$ & total number of protected hosts after a time interval of $t(j)$ \\
\hline
\end{tabular}




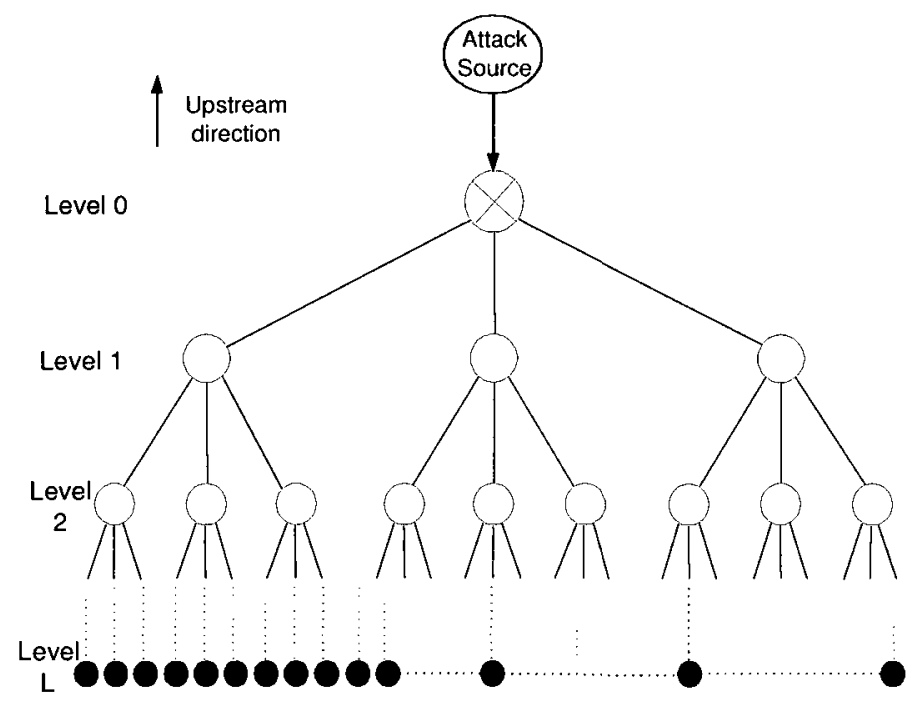

Figure 6.2: Hierarchical network topology used in AAWC modeling. $\delta=1$.

Considering the random scanning behavior of malicious worms and using (5.5), derived earlier in section 5.3.1.1, we develop expressions for the total number of hosts $M(j)$ protected as a result of EDANC collaborative containment action carried out by a router at level $j$ in an $L$-level network. Using notations in Table 6.1, it is assumed that $y$ is same for all upstream routers, $W$ is same for all cells and $s$ is same for all gateway routers. We realise that this assumption might not be applicable to all networks on the Internet, but we consider it sufficient to demonstrate the protection capability of EDANC collaborative containment. $M(j)$ can therefore be expressed as:

$$
M(j)=W s y^{L-j} \quad 0 \leq j \leq L
$$

Let us assume the worm's travel time from source to destination is negligible. This assumption is not unrealistic for fast propagating worms. If we also assume that the correlation time interval for the GEP theory based correlation engine is negligible $\ddagger$, then the time of containment $t(j)$ at any level $j$ in the hierarchical network for an intrusion with traffic profile

$\ddagger$ Processing speed of modern gateway routers make this assumption realistic. 


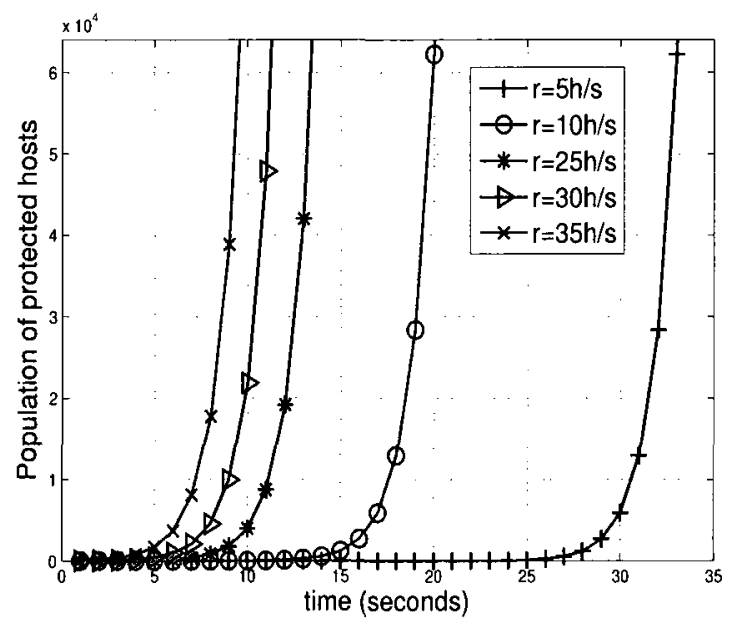

Figure 6.3: Effect of $r$ on protected population $M(j) . W=254, m=128$.

$i$ can be expressed as:

$$
t(j)=\frac{W+v_{i}}{2 r}+(L-j) \delta
$$

where $\delta$ is the notification time interval between neighbor routers in the network. Equation (6.2) comprises the average detection interval derived earlier in Section 5.3.1.1 and the collaborative containment interval based on our hierarchical network topology (Fig. 6.1 and Fig. 6.2). Solving for $L$ and substituting in (6.1), we get:

$$
M(j)=W s y^{\frac{t(j)-\frac{W+v_{i}}{2 r}}{\delta}} \quad 0 \leq j \leq L
$$

Using (6.3), Fig. 6.3 was generated to show the impact of varying worm scanning rate $r$ on $M(j)$. Fig. 6.3 shows that the protected population increases exponentially after the initial containment action on the gateway router due to collaborative containment. Also, an increase in worm scanning rate causes a faster EDANC defense response, hence a greater population is protected within a shorter time interval. 


\subsubsection{EDANC Protection Capability}

Using the AAWC model introduced in the previous section, we define the protection capability of the EDANC scheme in terms of the number of hosts an active worm successfully infects before further spread of the worm due to direct scans is completely contained. The smaller the number, the more effective the defense scheme is assumed to be. In order to quantitatively analyze EDANC protection capability using the AAWC model, it is important to first model malicious worm propagation. In this section, we briefly review the Analytical Active Worm Propagation (AAWP) model [19], a well known model for worm propagation and then adapt the AAWP model to our hierarchical network topology.

\subsubsection{Review of the AAWP Model}

Active worms often propagate by first randomly scanning a network, infecting vulnerable hosts in the network and then using infected hosts as a vehicle for further scanning and spreading. The Analytical Active Worm Propagation (AAWP) model [19] was chosen to model worm propagation in our analysis because it more accurately captures the behavior of random scanning worms compared to epidemiological models [19]. We explained this in better detail in Section 3.1. In addition, it is a discrete time model similar to our AAWC model. The AAWP model shows that the number of newly infected hosts in each time increment as a result of a random scanning worm attack is determined by parameters such as the size of the total population that the worm scans, the total number of vulnerable hosts in the population, the scanning rate of the worm, the patching rate, the death rate, and the time it takes for the worm to complete infection on a vulnerable host [19]. Using parameters in Table 6.2, our implementation of the AAWP model assumes that a worm randomly scans a class A network (with $2^{24}-2$ host IP addresses) $\S$ and requires one time increment to

\footnotetext{
$\S$ A class A network was chosen because most ISPs and large networks which are typical targets of large scale
} worm attacks have class A IP address blocks. 
Table 6.2: Parameters for AAWP Model

\begin{tabular}{|l|l|}
\hline Notation & Explanation \\
\hline$H$ & total number of vulnerable machines \\
\hline$r$ & scanning rate (the average number of machines scanned by an infected machine per unit time) \\
\hline$p$ & patching rate (the rate at which an infected or vulnerable machine becomes invulnerable) \\
\hline$z$ & size of hitlist (the number of infected machines at the beginning of the spread of active worms) \\
\hline$d$ & death rate (the rate at which an infection is detected on a machine and eliminated without patching) \\
\hline$n_{i}$ & number of infected machines at time tick $t_{i}$ \\
\hline$h_{i}$ & number of vulnerable machines (including infected ones) at time tick $t_{i}$ \\
\hline
\end{tabular}

infect a vulnerable host. Therefore, the probability that a host is hit by one scan is $\frac{1}{2^{24}}$. If at time tick $t_{0}$ there are $n_{0}=z$ infected hosts and $h_{0}$ vulnerable hosts then the effective initial scanning rate will be $z * r$ and there will be $\left(h_{1}-n_{1}\right)\left[1-\left(1-\frac{1}{2^{24}}\right)^{n_{1} r}\right]$ newly infected hosts on the next time tick, $t_{1}$. It was shown in [19] that with death rate $d$ and patching rate $p$, the total number of infected hosts $n_{i+1}$ at a time tick $t_{i+1}$ can be expressed as:

$$
n_{i+1}=n_{i}+\left(h_{i}-n_{i}\right)\left[1-\left(1-\frac{1}{2^{24}}\right)^{n_{i} r}\right]-(d+p) n_{i}
$$

Also, the total number of vulnerable hosts (including infected ones) reduce by a factor of $(1-p)$ after every time tick. Hence, $h_{i+1}=(1-p) h_{i}$ and $h_{i}=(1-p)^{i} h_{0}=(1-p)^{i} H$. Solving, $n_{i+1}$ can be expressed as:

$$
n_{i+1}=(1-d-p) n_{i}+\left[(1-p)^{i} H-n_{i}\right]\left[1-\left(1-\frac{1}{2^{24}}\right)^{n_{i} r}\right]
$$

where $i \geq 0, n_{0}=z$ and $h_{0}=H$. According to [19], the recursion stops when there are no remaining vulnerable hosts or when the worm can no longer increase the total number of infected hosts.

\subsubsection{AAWP Model in our Hierarchical Network}

We make the following assumptions in adapting the AAWP model to the hierarchical network topology. 
1. The entire host population in target networks are initially vulnerable to the worm attack.

2. Infection on infected hosts are eliminated only by patching. Hence, death rate $d=0$.

3. For analysis, we assume that one time tick is equivalent to one second.

Using the hierarchical network topology in Fig. 6.2, total number of vulnerable hosts, $H$ in the network can be expressed as:

$$
H=W s y^{L} \quad \text { derived from (6.1) using } j=0
$$

Substituting for $H$ in (6.4), $n_{i+1}$ can be expressed as:

$$
n_{i+1}=(1-p) n_{i}+\left[(1-p)^{i}\left(W s y^{L}\right)-n_{i}\right]\left[1-\left(1-\frac{1}{2^{24}}\right)^{n_{i} r}\right]
$$

In our analysis, we assume that the attacking worm is a fast scanning malicious worm. The adapted AAWP model (6.5) is used to model the number of hosts infected as a result of the worm attack while the AAWC model (6.3) is used to model the number of hosts protected as a result of the EDANC scheme.

Fig. 6.4 shows that while the AAWP model shows steady growth in infected population with time, the AAWC model shows an even greater growth in the number of protected hosts due to collaborative containment in the network. A perimeter is created on a gateway router or upstream router after a containment action is taken thus preventing further direct scans. For a scanning worm attack, successful direct worm scans are stopped when the number of protected hosts (modeled using AAWC model) exceed the number of directly scanned hosts (modeled using AAWP model), thus preventing further increase in the number of hosts infected by direct scans (see Fig. 6.4). Fig. 6.4 shows that this is achievable within 13 seconds after release of the worm. 


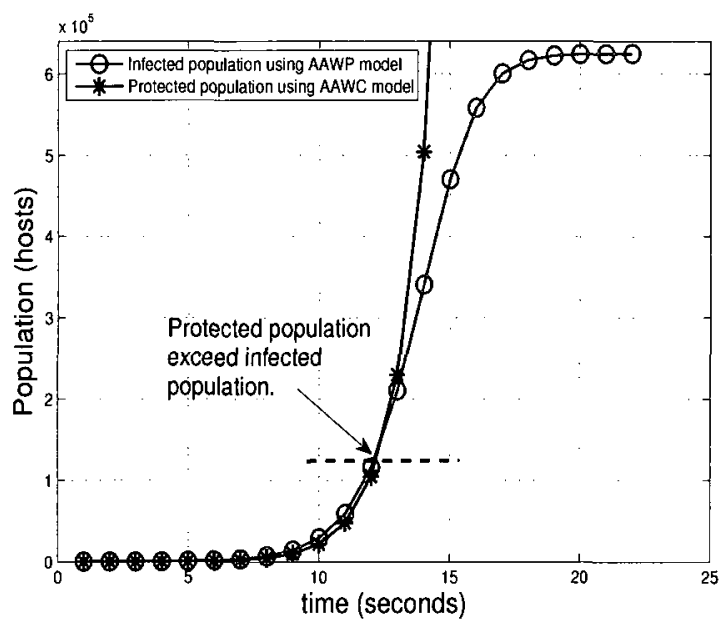

Figure 6.4: AAWC vs. AAWP. $z=30, y=3, s=3, L=6, p=0, r=30, \delta=1$.

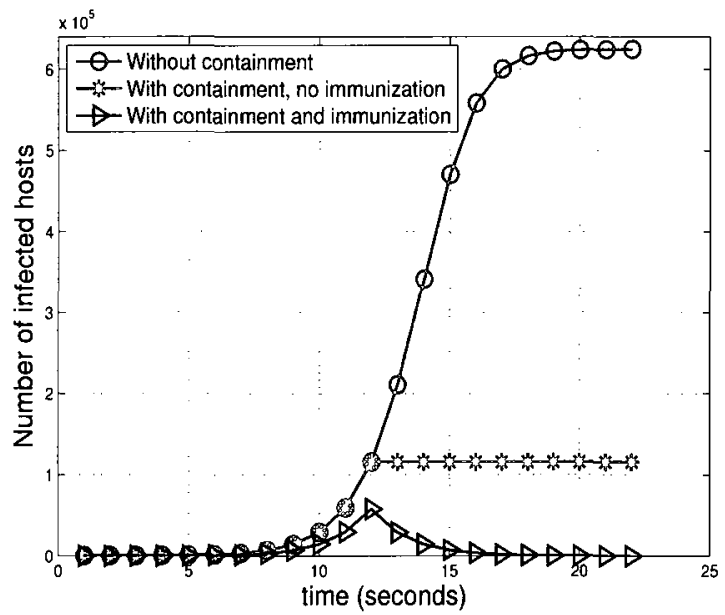

Figure 6.5: Effect of network containment and immunization on infected population. $z=$ $30, y=3, s=3, L=6, p=0.3, r=30, \delta=1$.

\subsubsection{EDANC with Immunization}

Immunization by quickly deploying patches on infected hosts has been proposed as an effective defense strategy for worms [86] [8]. Worm defense using EDANC can effectively and quickly stop further direct worm scans as shown in Fig. 6.4, but does not address the infectious state of hosts infected before complete containment of direct worm attacks. It also 
does not defend against local scanning within a protected cell that contains an infected host. For complete eradication of infection we introduce immunization to the EDANC scheme. For this analysis, a non-zero patching rate, $p=0.3$ is introduced in the AAWP model (6.5). This causes the number of infected hosts $n_{i}$ at time $t_{i}$ to decrease by the patching rate on every subsequent time tick. Fig. 6.5 shows that the number of infected hosts in protected cells is significantly reduced and tends towards zero as a result of this combined approach thus ensuring that hosts infected before complete containment of direct worm attacks do not become launching platforms for more attacks within or outside their cells.

\subsection{Chapter Summary}

In this chapter, considering the random scanning behavior of malicious worms, we developed the Analytical Active Worm Containment (AAWC) model, a discrete-time model used to model vulnerable host population protected as a result of the EDANC scheme in a large scale network. Using the AAWC model, we demonstrated quantitatively the collaborative containment capability of EDANC. For a scanning worm attack, the AAWC model assumes that successful direct worm scans are stopped when the number of protected hosts (modeled using AAWC model) exceed the number of directly scanned hosts (modeled using the known AAWP model [19]), thus preventing further increase in the number of hosts infected by direct scans. Our results showed that this is achievable within 13 seconds after release of the worm.

In addition, it was observed that while network containment of worms can halt further worm spread due to direct scans, it does not recover infected hosts nor does it prevent

local scanning within a protected cell. Immunization by patching was therefore introduced to EDANC by introducing a non-zero patching rate to the AAWP model. This combined approach caused the number of infected hosts after EDANC defense to decrease by the patching rate on every subsequent time tick. Results showed that the number of infected 
hosts in protected cells was significantly reduced and tended towards zero as a result of this combined approach, thus ensuring that hosts infected before EDANC defense did not become launching platforms for more attacks within or outside their network cells. 


\section{Chapter 7}

\section{Integrated Detection using EDANC}

\subsection{Introduction}

The propagation speed of fast scanning worms and the stealthy nature of slow scanning worms present unique challenges to intrusion detection. Typically, techniques optimized for detection of fast scanning worms fail to detect slow scanning worms, and vice versa. Typically, malicious traffic flows of varying scanning rates can occur concurrently on computer networks, hence there is interest in developing an integrated approach to detecting both types of worms. Detection of slow worms is particularly challenging due to the stealthy nature of their propagation techniques and their ability to blend with normal traffic patterns.

In this chapter, we introduce improvements to our earlier proposed EDANC detection technique (presented in Chapter 5) which extend its capabilities to slow worm detection. Using the EDANC technique we present an integrated approach for detecting both fast and slow scanning malicious worms. The integrated detection approach is also based on the Generalized Evidence Processing (GEP) theory, a multi-sensor data fusion technique [72] [73]. With GEP theory, evidence collected by distributed detectors, running host based anomaly 
detection software determine the probability associated with a detection decision under a hypothesis. The probability mass associated with each host detector decision is combined in a correlation engine to arrive at an improved fused detection decision by minimizing a cummulative decision risk function.

In practice, malicious traffic flows of different scanning rates can occur concurrently in computer networks, and the difficulty in detecting slow scanning worms in particular can be exacerbated by interference from other traffic flows scanning at faster rates. The integrated detection technique uses a window-based self adapting profiler to filter detected fast scanning malicious traffic profiles hence isolating slow scanning malicious traffic profiles in the correlation engine.

The main contributions in this chapter are:

$\dagger$ We propose an integrated approach to detecting both fast and slow scanning malicious worms using the EDANC scheme.

† Our approach takes into consideration the real possibility that faster propagating malicious intrusions can co-exist with slow worms in computer networks, and therefore interfere with slow worm detection.

$\dagger$ We achieve detection of slow malicious worms in the presence of fast scanning worms by using a combination of detector decisions from host-based anomaly detectors, a window-based adaptive profiler and correlation engines based on the Generalized Evidence Processing (GEP) theory.

$\dagger$ Experimenting on a live test-bed we demonstrate the technique and present results. 


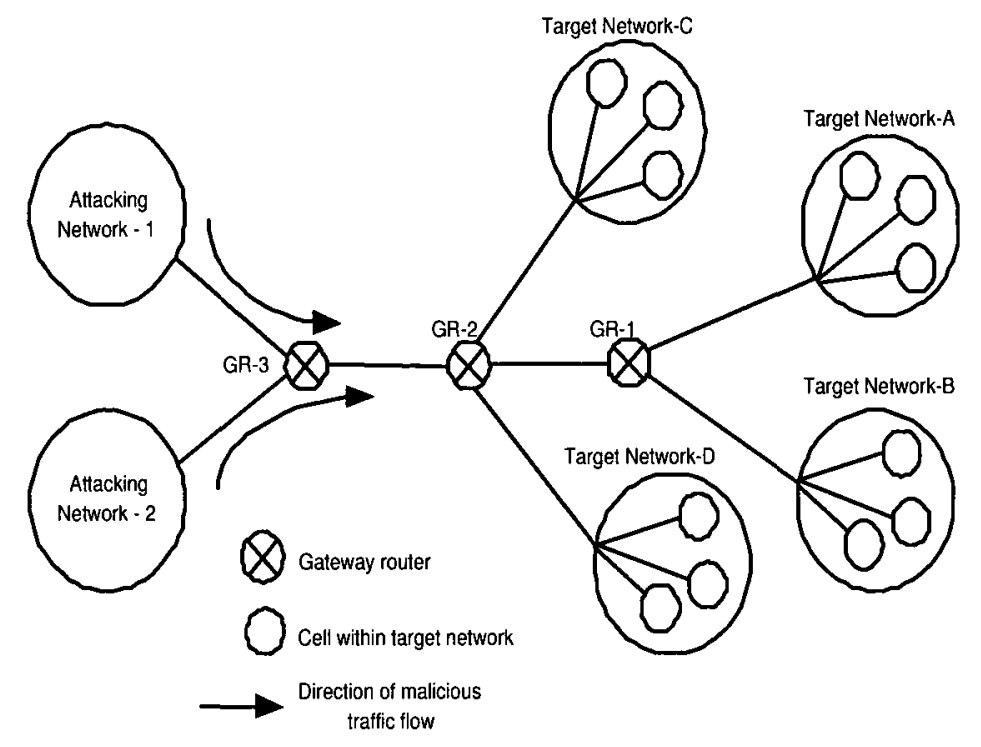

Figure 7.1: Typical worm attack on multiple networks

\subsection{Integrated Detection Approach}

Fig. 7.1 depicts a typical attack scenario in which single or multiple attackers in Network1 and Network-2 launch scanning worm attacks on several enterprise networks (Target Network-A, Target Network-B, Target Network-C, Target Network-D). Typically, well-designed enterprise networks are logically subdivided into cells or network zones as shown in Fig. 7.1. The detection scheme depends on detector endpoints within distributed cells in a target network for detecting intrusion attempts and making local decisions about the traffic flows that cause the intrusion attempts. The detector local decisions and captured traffic logs are combined at the gateway router (which is the fusion center) of the cells using our improved correlation technique to arrive at a fused decision. 
Table 7.1: Detection Parameters

\begin{tabular}{|l|l|}
\hline Notation & Explanation \\
\hline$S W_{j}$ & $j^{t h}$ slow worm detection window \\
\hline$F W_{k j}$ & $k^{t h}$ fast worm detection window within $S W_{j}$ \\
\hline$t_{s}$ & duration of slow worm detection window \\
\hline$t_{f}$ & duration of fast worm detection window \\
\hline$Z_{j}$ & set of profiles captured by the SWDA during $S W_{j}$ \\
\hline$X_{k j}$ & worm profiles detected during the $F W_{k j}$ window \\
\hline$Y_{j}$ & set of profiles forwarded to the slow worm correlation engine (SWCE) \\
\hline$d_{f q}^{i}$ & Individual decision by FWDA on the $q^{t h}$ DE on intrusion attempts due to profile $i$. \\
\hline$d_{s q}^{j}$ & Individual decision by SWDA on the $q^{t h}$ DE on intrusion attempts due to profile $j$. \\
\hline
\end{tabular}

\subsubsection{Detection Technique}

Our technique uses two instances of detector agents, fast worm detector agent (FWDA) and slow worm detector agent (SWDA). Both run simultaneously on detector endpoints (DEs) located within distributed cells in the network and are responsible for capturing malicious intrusion attempts targeted at the cells. The detector agents run host-based anomaly detection software configured to capture intrusion data * when malicious intrusions are detected on the detector endpoints. While the SWDA is used for detection of fast and slow propagating malicious worms, the FWDA is used for detecting worm intrusions that are fast propagating. This is achieved by capturing intrusion data during two different detection time window intervals. Table 7.1 and Fig. 7.2 describe some of the detection algorithm parameters and notations used in our explanation below.

\subsubsection{Intrusion Detection Windows $\left(F W_{k j}, S W_{j}\right)$}

We refer to an epoch that spans a capture interval as a detection window. Two detection windows are used in our detection algorithm - the "fast worm detection window" $\left(F W_{k j}\right)$ and the "slow worm detection window" $\left(S W_{j}\right)$. A fast worm detection window refers to an epoch of duration $t_{f}$ started as a result of an intrusion attempt detected by a FWDA. A slow

* As explained earlier in Section 5.4.1, intrusion data includes traffic profiles hits on the DE, transfer logs and existence of the transfered file on the DE. 


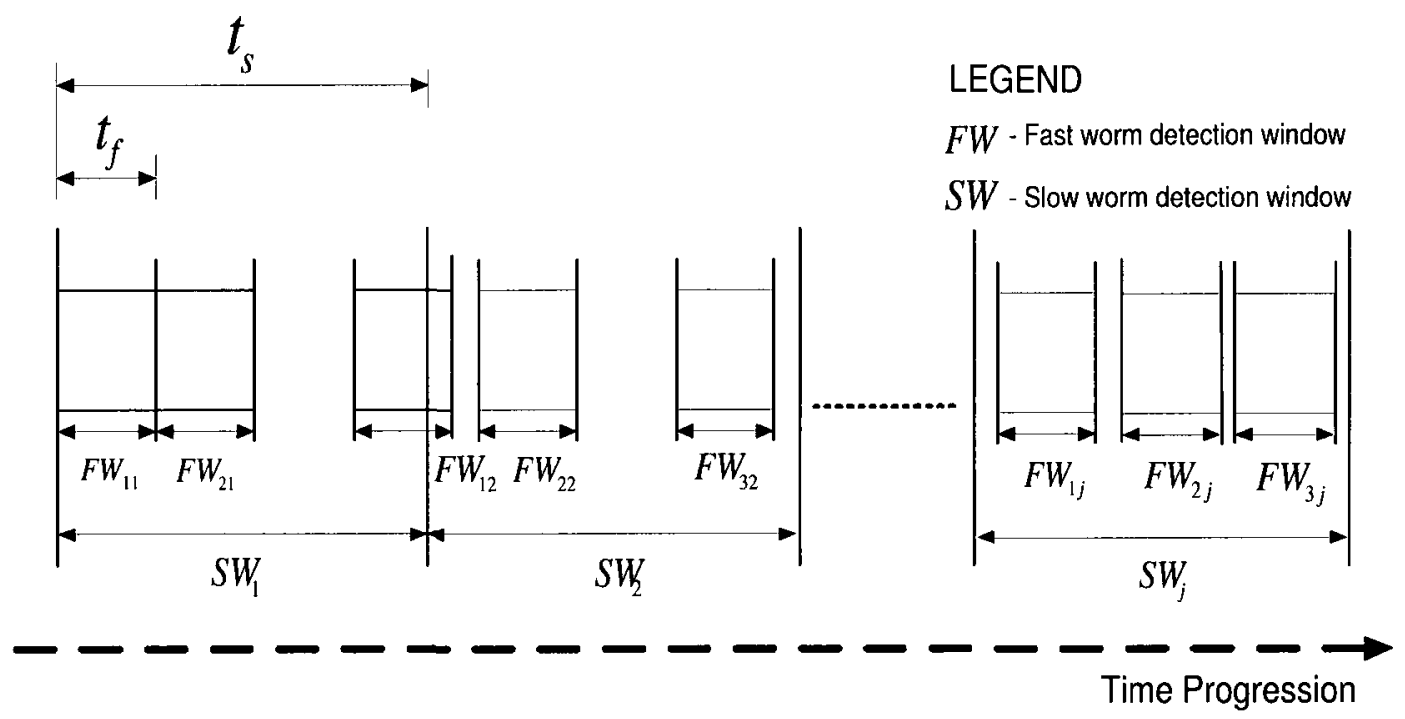

Figure 7.2: Series of epochs showing detection windows

worm detection window refers to a periodic epoch of duration $t_{s}$ which runs continuously on each SWDA. Fig. 7.2 shows a snapshot of a series of epochs during which the FWDA and SWDA carry out real-time recording of incoming network traffic profiles. Typically, $t_{s}>t_{f}$, hence there could be multiple fast worm detection windows within a single slow worm detection window (Fig. 7.2). At the end of a fast worm detection window, all profiles recorded by a FWDA running on a DE in the cell are transferred to the first upstream gateway router for correlation. After the transfer, the FWDA continues to monitor for future intrusion attempts. The SWDAs wait until the end of the slow worm detection window before transfering captured records to the gateway router. The next slow worm detection window is started immediately after the transfer.

\subsubsection{Fast Worm Detection}

When a FWDA running on a detector endpoint (DE) detects an unauthorized intrusion associated with a particular traffic profile, the following occurs: 
1. The FWDA immediately starts real-time recording of profiles of all network traffic originated from outside its cell and targeted at the DE for a pre-set capture interval. The FWDA capture interval corresponds to the fast worm detection window with duration $t_{f}$.

2. The FWDA also immediately sends an alert to other participating FWDAs within the cell. FWDAs communicate only with other FWDAs.

3. When the alert is received, the FWDAs within the target cell also start real-time recording of profiles for all network traffic originated from outside their cell and targeted at the DEs for the pre-set capture interval.

4. For each traffic profile $i$ detected in the target cell by a FWDA, two hypotheses $H_{1}$ and $H_{0}$ are considered, where $H_{1}$ is the hypothesis that the traffic profile $i$ is malicious and $H_{0}$ is the hypothesis that the traffic profile $i$ is benign. For the profile $i$, let $d_{f q}^{i}$ be the individual local decision by the FWDA on the $q^{\text {th }}$ DE based on observed intrusion attempts. $d_{f q}^{i}=1$ if $H_{1}$ is decided and $d_{f q}^{i}=0$ if $H_{0}$ is decided. We assume the anomaly detection software running on a FWDA is capable of making such a decision. For this work, we considered a binary local detection outcome which did not include indecision, though the GEP theory is capable of dealing with indecision as explained earlier in Chapter four. For a target cell with $m$ DEs, let $\underline{d}_{f}^{i}=\left(d_{f 1}^{i}, d_{f 2}^{i}, . ., d_{f m_{i}}^{i}\right)$ be the vector of individual FWDA decisions on traffic profile $i$.

5. At the end of the fast worm detection window, the FWDAs on all DEs in the cell transfer their records and local decisions to their upstream gateway router and continue monitoring the DEs for unauthorized intrusions. 


\subsubsection{Slow Worm Detection}

As mentioned earlier, we assume both SWDA and FWDA use the same anomaly detection mechanism, though the SWDA records network traffic profiles for a longer period, $t_{s}$. The SWDAs perform continuous real-time capturing of profiles of all network traffic originated from outside their cell and targeted at the DEs in epochs of interval $t_{s}$ which corresponds to the slow worm detection window. During a slow worm detection window, the following occurs:

1. For each traffic profile $j$ detected in the target cell by a SWDA, two hypotheses $H_{1}$ and $H_{0}$ are considered, where $H_{1}$ is the hypothesis that the traffic profile $j$ is malicious and $H_{0}$ is the hypothesis that the traffic profile $j$ is benign. For the profile $j$, let $d_{s q}^{j}$ be the individual local decision by the SWDA on the $q^{\text {th }}$ DE based on observed intrusion attempts. $d_{s q}^{j}=1$ if $H_{1}$ is decided and $d_{s q}^{j}=0$ if $H_{0}$ is decided. We assume the anomaly detection software running on a SWDA is capable of making such a decision. For this work, we considered a binary local detection outcome which did not include indecision, though the GEP theory is capable of dealing with indecision as explained earlier. For a target cell with $m$ DEs, let $\underline{d}_{s}^{j}=\left(d_{s 1}^{j}, d_{s 2}^{j}, . ., d_{s m_{j}}^{j}\right)$ be the vector of individual SWDA decisions on traffic profile $j$.

2. At the end of a slow worm detection window, the SWDAs on all DEs in the cell transfer their records and local decisions to their first upstream gateway router and immediately start the next epoch of recording.

Unlike the FWDAs, the SWDAs do not wait for an alert before capturing intrusion data. Intrusion data is captured in periodic slow worm detection windows of duration $t_{s}$. 
Table 7.2: Parameters for Integrated GEP-based correlation algorithm

\begin{tabular}{|l|l|}
\hline Notation & Explanation \\
\hline$P_{D i}^{f}$ & Combined probability of detection for traffic profile $i$ on FWCE \\
\hline$P_{D j}^{s}$ & Combined probability of detection for traffic profile $j$ on SWCE \\
\hline$p_{d q}^{f}$ & Detection probability for the FWDA on the $q^{t h}$ DE \\
\hline$p_{f q}^{s}$ & False detection probability for the FWDA the $q^{t h}$ DE \\
\hline$p_{d q}^{f}$ & Detection probability for the SWDA on the $q^{t h}$ DE \\
\hline$p_{f q}^{s}$ & False detection probability for the SWDA the $q^{t h}$ DE \\
\hline$d_{f q}^{i}$ & Individual decision by FWDA on the $q^{t h}$ DE on intrusion attempts due to profile $i$. \\
\hline$d_{s q}^{j}$ & Individual decision by SWDA on the $q^{t h}$ DE on intrusion attempts due to profile $j$. \\
\hline $\mathbf{D}^{f}$ & Fused decision on FWCE. \\
\hline $\mathbf{D}^{\mathbf{s}}$ & Fused decision on SWCE. \\
\hline$\Lambda\left(d_{f}^{i}\right)$ & GEP likelihood ratio for optimized fused decision on FWCE about profile $i$ \\
\hline$\Lambda\left(d_{s}^{j}\right)$ & GEP likelihood ratio for optimized fused decision on SWCE about profile $j$ \\
\hline$C_{a b}^{f}$ & Cost associated with a detector decision $a$ when the true hypothesis is $H_{b}$ on FWCE \\
\hline$C_{a b}^{s}$ & Cost associated with a detector decision $a$ when the true hypothesis is $H_{b}$ on SWCE \\
\hline$\gamma_{f}$ & GEP likelihood ratio threshold on the FWCE, also equivalent to $\frac{C_{10}^{f}}{C_{01}^{f}}$ on the FWCE \\
\hline$\gamma_{s}$ & GEP likelihood ratio threshold on the SWCE, equivalent to $\frac{C_{10}^{s}}{C_{01}^{s}}$ on the SWCE \\
\hline$m_{i}^{f}$ & Total number of FWDAs with observations of profile $i$ \\
\hline$u_{i}^{f}$ & Total number of FWDAs with observations of profile $i$ and that favor $H_{1}$ \\
\hline$v_{i}^{f}$ & Number of FWDAs which favor $H_{1}$ required to minimally satisfy $\Lambda\left(d_{f}{ }^{i}\right) \geq \gamma$ \\
\hline$m_{j}^{s}$ & Total number of SWDAs with observations of profile $j$ \\
\hline$u_{j}^{s}$ & Total number of SWDAs with observations of profile $j$ and that favor $H_{1}$ \\
\hline$v_{j}^{s}$ & Number of SWDAs which favor $H_{1}$ required to minimally satisfy $\Lambda\left(d_{s}^{j}\right) \geq \gamma$ \\
\hline
\end{tabular}

\subsubsection{Correlation Technique}

The upstream gateway router receives the records and local decisions transfered from the FWDAs and SWDAs on DEs in the target cell. The gateway router runs two correlation engines, Fast Worm Correlation Engine (FWCE) which executes a Fast Worm Correlation Algorithm (FWCA) and a slow worm correlation engine (SWCE) which executes a slow worm correlation algorithm (SWCA). Fig. 7.3 shows the communication flow between the DEs and the gateway router correlation engines. Both FWCA and SWCA use the GEP theory evidence combining technique to determine the profile(s) associated with the detected malicious or unauthorized intrusion(s). Multiple correlation processes can run on the gateway router simultaneously. 


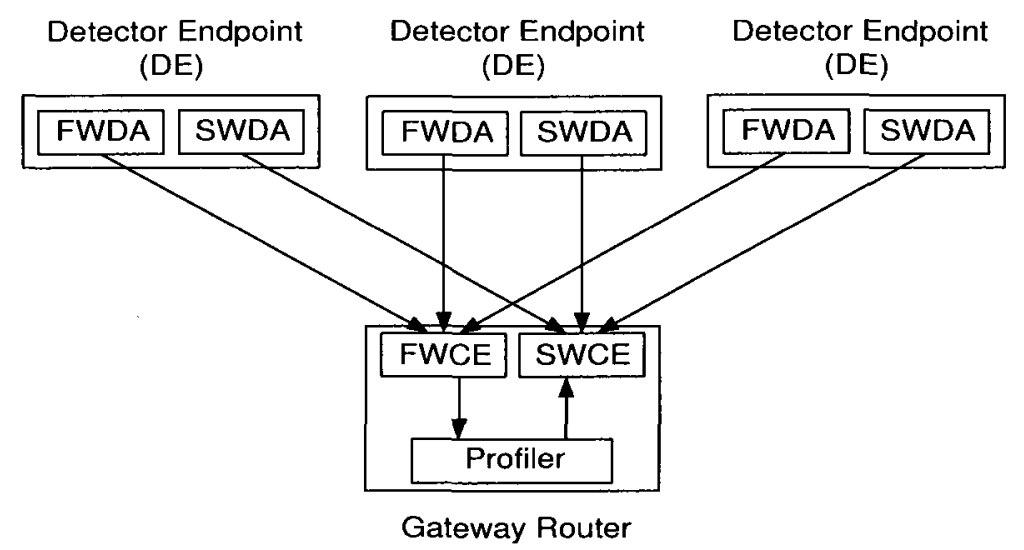

Figure 7.3: Communication flow between Detector Endpoints and Gateway Router

\subsubsection{Fast Worm Correlation Algorithm (FWCA)}

At the gateway router, we are interested in using collected FWDA local decisions in making an improved fused decision which minimizes the cummulative decision risk of making a decision. For each traffic profile $i$ with associated records and local decisions received from the FWDAs, two hypotheses $H_{1}$ and $H_{0}$ are considered at the gateway router, where $H_{1}$ is the hypothesis that the traffic profile $i$ is malicious and $H_{0}$ is the hypothesis that the traffic profile $i$ is benign. As explained in Section 5.2.2.1, the GEP optimized decision criteria at the fusion centre (the gateway router) which minimizes the cummulative decision risk can be expressed using the following likelihood ratio rule (derived from (4.5)):

$$
\Lambda\left(\underline{d_{f}^{i}}\right)=\frac{P\left(\underline{d}_{f}^{i} \mid H_{1}\right)}{P\left(\underline{d}_{f}^{i} \mid H_{0}\right)} \underset{\mathbf{D}^{\mathbf{f}=0}}{\stackrel{\mathbf{D}^{\mathbf{f}}=1}{\gtrless}} \frac{C_{10}^{f}}{C_{01}^{f}}=\gamma_{f}
$$

where $C_{10}^{f}$ and $C_{01}^{f}$ are the costs of a fused decision that results in a false positive and a false negative respectively on the FWCE. As explained in Section 5.2.2.1, a $\gamma_{f}=\frac{C_{10}}{C_{01}}$ value of 2.0 was used in our experiments with fast worm detection to ensure that a greater cost or penalty was associated with a decision that results in a false positive than a decision that results in a false negative. 
For homogeneous detectors, $p_{d q}^{f}=p_{d}^{f}$ and $p_{f q}^{f}=p_{f}^{f}$, for all $q$. Therefore, the likelihood ratio test in (7.1) can be expressed as:

$$
\Lambda\left(\underline{d_{f}^{i}}\right)=\frac{P\left(\underline{d}_{f}^{i} \mid H_{1}\right)}{P\left(\underline{d}_{f}^{i} \mid H_{0}\right)}=\left(\frac{p_{d}^{f}}{p_{f}^{f}}\right)^{u_{i}} *\left(\frac{1-p_{d}^{f}}{1-p_{f}^{f}}\right)^{m_{i}^{f}-u_{i}^{f}} \underset{\mathbf{D}^{\mathbf{f}}=0}{\underset{\mathbf{D}}{\mathbf{f}=1}} \frac{C_{10}^{f}}{C_{01}^{f}}=\gamma_{f}
$$

By computing for $\Lambda\left(\underline{d_{f}^{i}}\right)$, the likelihood ratio test in (7.2) determines whether the correlation algorithm considers traffic profile $i$ as a malicious traffic profile (i.e. fused decision $\mathbf{D}^{\mathbf{f}}=1$ ) or a benign traffic profile (i.e. fused decision $\mathbf{D}^{\mathbf{f}}=0$ ).

As explained in Section 5.2.2.1, when multiple simultaneous malicious fast worm attacks occur and are detected using the correlation algorithm, the combined probability of detection, $P_{D i}^{f}$ for each malicious profile $i$ is computed. $P_{D i}^{f}$ can be expressed as:

$$
P_{D i}^{f}=P\left(\Lambda\left(\underline{d_{f}^{i}}\right) \geq \gamma_{f} \mid H_{1}\right)=\sum_{u_{i}^{f}: u_{i}^{f} \geq v_{i}^{f}}\left(\begin{array}{c}
m_{i}^{f} \\
u_{i}^{f}
\end{array}\right)\left(p_{d}^{f}\right)^{u_{i}^{f}}\left(1-p_{d}^{f}\right)^{m_{i}^{f}-u_{i}^{f}}
$$

Containment action for the multiple malicious traffic profiles is triggered in a sequence ordered by the value of $P_{D i}^{f}$. Containment for profile $u$ is triggered before that of profile $v$ if,

$$
P_{D u}^{f}>P_{D v}^{f}
$$

\subsubsection{Window-based profiler}

Let $F W_{k j}$ be the $k^{t h}$ fast worm detection window within the $j^{\text {th }}$ slow worm detection window $S W_{j}$ (see Table 7.1 and Fig. 7.2). We use $X_{k j}$ to model a set with elements corresponding to profile(s) identified as associated with fast scanning worm intrusion(s). These profiles are 


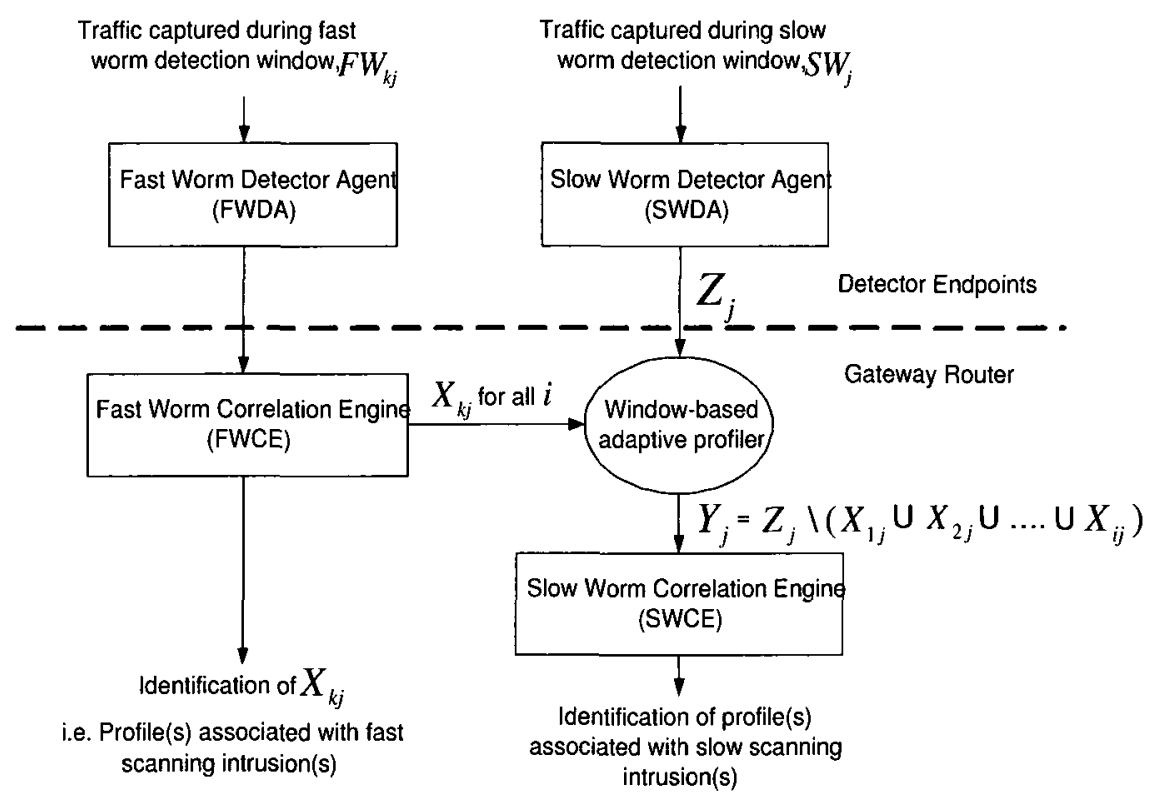

Figure 7.4: Block diagram of integrated detection of fast and slow worms.

identified by the FWCE from records captured during $F W_{k j}$ (see Fig. 7.4). We also use $Z_{j}$ to model the set with elements corresponding to all profiles captured by the SWDAs in the cell during the slow worm detection window $S W_{j}$. As mentioned earlier, we assume the SWDAs run that same host anomaly detection software as the FWDAs but record traffic profiles for a longer period, $t_{s}$ which corresponds to the slow worm detection window $S W_{j}$. For each slow worm detection window, the window-based adaptive profiler tags traffic profiles identified by the FWCE and periodically adapts the input into the slow worm correlation engine (SWCE) by filtering out the tagged profiles. This ensures that only profiles that have not been previously identified by the FWCE as associated with faster propagating intrusions are forwarded to the SWCE (Fig. 7.4). We used a similar adaptive profiler technique in [5] to filter out traffic profiles belonging to fast scanning worms. If $Y_{j}$ is the set with elements corresponding to profiles forwarded to the SWCE, then $Y_{j}$ is expressed as:

$$
Y_{j}=Z_{j} \backslash\left(X_{1 j} \cup X_{2 j} \cup \ldots \ldots \cup X_{k j}\right)
$$


This window-based adaptive profiler algorithm ensures that for every slow worm detection window, $S W_{j}$, the corresponding $Y_{j}$ is updated with outputs, $X_{k j}$ from the FWCE. At the end of a slow worm detection window, only profiles that are not deemed to belong to faster propagating intrusions by the FWCE are forwarded to the SWCE for slow worm detection and identification. The SWCE runs the slow worm correlation algorithm (SWCA) described in the next section.

\subsubsection{Slow Worm Correlation Algorithm (SWCA)}

The SWCE runs the slow worm correlation algorithm (SWCA) on $Y_{j}$. Slow scanning worms are known to exhibit high rates of false negatives since they are capable of avoiding detection by scanning at rates below most traditional IDS thresholds and blending with normal traffic patterns. As a result, unlike fast worms they inherently exhibit greater false negative rates than false positive rates. We use $\gamma_{s}=\frac{C_{10}^{s}}{C_{01}^{g}}$ where $0 \leq C_{a b}^{s} \leq 1$ to denote the decision cost ratio used for slow worm detection in the SWCA. We also use $\underline{d_{s}}{ }^{j}$ to denote the vector of local decisions from the SWDAs corresponding to the elements in $Y_{j}$. The slow worm correlation algorithm (SWCA) detects slow worm profiles by using the following likelihood ratio rule (derived from (4.5)):

$$
\Lambda\left(\underline{d_{s}^{j}}\right)=\frac{P\left(\underline{d}_{s}^{j} \mid H_{1}\right)}{P\left(\underline{d}_{s}^{j} \mid H_{0}\right)} \underset{\mathbf{D}=0}{\stackrel{\mathrm{D}=1}{\gtrless}} \gamma_{s}=\frac{C_{10}^{s}}{C_{01}^{s}}
$$

where $C_{01}^{s}>C_{10}^{s}$, thus ensuring a greater penalty for false negatives than false positives. Parameter $C_{10}^{s}$ and $C_{01}^{s}$ are the costs of a fused decision that results in a false positive and a false negative respectively on the SWCE. Unlike fast scanning worms, malicious slow worms are more likely to go undetected in the network since they can camouflage as normal traffic. We therefore consider false negatives more severe than false positives for slow worms and associate a greater cost or penalty for false negatives than false positives in our experiments 
with slow worm detection. We used $\gamma_{s}=\frac{C_{10}^{s}}{C_{01}^{s}}=0.1$ in the experiments.

For homogeneous detectors, $p_{d q}^{s}=p_{d}^{s}$ and $p_{f q}^{s}=p_{f}^{s}$, for all $q$. Therefore, the likelihood ratio test in (7.6) can be expressed as:

$$
\Lambda\left(\underline{d_{s}^{j}}\right)=\frac{P\left(\underline{d}_{s}^{j} \mid H_{1}\right)}{P\left(\underline{d}_{s}^{j} \mid H_{0}\right)}=\left(\frac{p_{d}^{s}}{p_{f}^{s}}\right)^{u_{j}^{s}} *\left(\frac{1-p_{d}^{s}}{1-p_{f}^{s}}\right)^{m_{j}^{s}-u_{j}^{s}} \underset{D^{s}=0}{\stackrel{D^{\mathbf{s}}=1}{\gtrless}} \frac{C_{10}^{s}}{C_{01}^{s}}=\gamma_{s}
$$

Given fixed parameters $p_{d}^{s}$ and $p_{f}^{s}$, the choice of $\gamma_{s}$ determines the minimum number of positive detectors (i.e. SWDAs) required to satisfy (7.7).

Note that our technique can also be used for detecting stealthy worms that are not necessarily scanning worms as long as such worms infect at least the minimum number of hosts required to satisfy (7.7) within the $S W_{j}$ window.

\subsection{Experimentation and Analysis}

\subsubsection{Description of test-bed setup}

Fig. 7.5 shows the topology of our live testbed. Worm attacks are sourced from Network-1 and Network-2 and targeted at vulnerable hosts in Network-A, Network-B, Network-C and Network-D. Detector endpoints (DEs) that run our detection algorithm are located within the target cells and communicate with their gateway routers (GR-1 and GR-2). The gateway routers runs our GEP-based correlation algorithm for the integrated EDANC scheme.

To evaluate the functionality and performance of our integrated detection scheme, we emulated self propagating worm attacks using a modified blaster worm source code [52]. To emulate multiple malicious attacks, the source code was used to instrument two worms that 


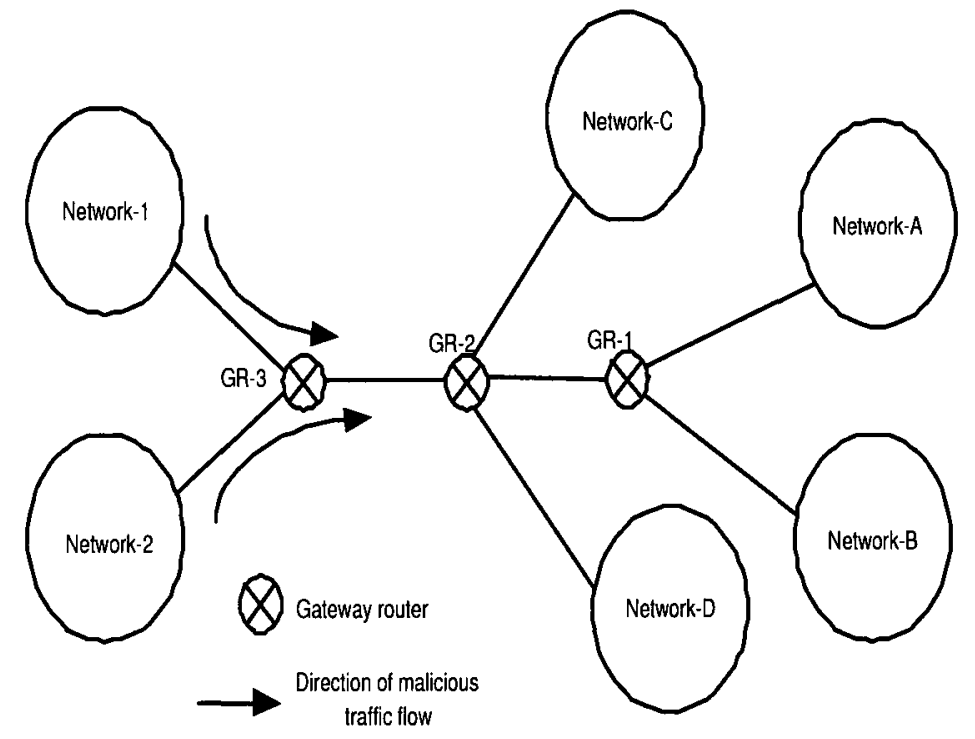

Figure 7.5: Test-bed used for experimentation

performed two different actions on the target hosts. The first, worm-1 was instrumented to create a directory named / root/infected-1 on the target host and copy a file named malicious1 into that directory over TCP port 888 . The second, worm- 2 was instrumented to create a directory named /root/infected- 2 on the target host and copy a file named malicious- 2 into that directory over UDP port 999. Hosts in Network-1 and Network-2 were used to launch worm-1 and worm-2 random attacks respectively on hosts in the target networks (Network A and Network B). We emulated slow worms with scanning rates of 5 hosts per minute $(\mathrm{h} / \mathrm{m})$ and $10 \mathrm{~h} / \mathrm{m}$ for our experiments. Slow worm rates and thresholds in the order of this magnitude have been used in previous works [65] [22]. Fast worms were emulated with scanning rates of 15 hosts per second $(\mathrm{h} / \mathrm{s})$ and $20 \mathrm{~h} / \mathrm{s}$. We used OpenVZ virtualization ${ }^{\dagger}$ [69] to create vulnerable host population in the target networks. Up to 128 virtual hosts per workstation were created on a number of Linux workstations running OpenVZ kernel-2.6.22 to emulate a vulnerable population in each target network.

$\dagger^{\dagger}$ OpenVZ is an operating system-level virtualization technology based on the Linux kernel and operating system. 
For our test, the host-based Anomaly Intrusion Detection System (AIDS) running on the Fast Worm Detector Agent (FWDA) and the Slow Worm Detector Agent (SWDA) were created using different instances of Linux-based scripts that constantly monitored the directory structure and content of the DE as well as the file transfer logs on the DE. Instances of Snort-based IDS running on the host detectors were used for real-time logging on the FWDA and SWDA. The FWDA and SWDA generate an alert when a file named malicious1 or malicious- 2 is transfered into a directory named/root/infected-1 or $/$ root/infected- 2 respectively on the DE. Fig. 5.2 and Fig. 5.3 in Section 5.2.2.1 show block diagrams of our implementation of host detection and alerting. Table 7.3 shows the relationship between $H_{1}, H_{0}$ and $p_{d}^{f}, p_{f}^{f}$ for each FWDA while Table 7.4 shows the relationship between $H_{1}, H_{0}$ and $p_{d}^{s}, p_{f}^{s}$ for each SWDA.

Table 7.3: Relationship between $H_{1}, H_{0}$ and $p_{d}^{f}, p_{f}^{f}$ for FWDA

\begin{tabular}{ccr}
\hline \hline True Nature & \multicolumn{2}{c}{ Local FWDA decision } \\
\hline$H_{1}$ & $H_{1}$ & $H_{0}$ \\
& $p_{d}^{f}$ & $1-p_{d}^{f}$ \\
$H_{0}$ & $p_{f}^{f}$ & $1-p_{f}^{f}$ \\
\hline
\end{tabular}

Table 7.4: Relationship between $H_{1}, H_{0}$ and $p_{d}^{s}, p_{f}^{s}$ for SWDA

\begin{tabular}{ccr}
\hline \hline True Nature & \multicolumn{2}{l}{ Local SWDA decision } \\
\hline & $H_{1}$ & $H_{0}$ \\
$H_{1}$ & $p_{d}^{s}$ & $1-p_{d}^{s}$ \\
$H_{0}$ & $p_{f}^{s}$ & $1-p_{f}^{s}$ \\
\hline
\end{tabular}

The parameter $t_{f}$ was set to 20 seconds on the FWDA and $t_{s}$ was set to 10 minutes on the SWDA. The gateway routers, GR-1 and GR-2 ran instances of the Fast Worm Correlation Engine (FWCE) and Slow Worm Correlation Engine (SWCE).

The purpose of the experiments was to demonstrate the functionality of the integrated detection technique in detecting both fast and slow propagating worm attacks even when they occur concurrently in the same computer network. The experiments may not be represen- 
tative of all the possible worm attack scenarios that exist on the Internet today.

For data generation and analysis of results we are interested in, each experiment was run 30 times and the average value as well as a confidence interval based on a $95 \%$ confidence level was computed.

Table 7.5: Worm attack sources and scanning rates used in Experiment 1

\begin{tabular}{crr}
\hline \hline Worm type & \multicolumn{2}{c}{ Worm sources and scanning rates } \\
\hline & worm-1 from Network-1 & worm-2 from Network-2 \\
Fast worms & $20 \mathrm{~h} / \mathrm{s}$ & $15 \mathrm{~h} / \mathrm{s}$ \\
Slow worms & $10 \mathrm{~h} / \mathrm{m}$ & $5 \mathrm{~h} / \mathrm{m}$ \\
\hline
\end{tabular}

\subsubsection{Experiment 1: Integrated detection of fast and slow worms}

In this experiment, four attacking hosts, two from Network-1 and two from Network-2 in Fig. 7.5 were used to launch different attacks (worm-1 and worm-2 respectively) on hosts in the target networks. Table 7.5 shows the sources and scanning rates of the worms used in this experiment. The objective of the experiment was to demonstrate the functionality of our integrated detection technique when fast and slow worms occur concurrently in a target network.

Fig. 7.6 shows that the number of detectors (i.e. FWDAs) that positively detected the fast worms exceeded the number required for a fused decision $\mathbf{D}^{\mathbf{f}}=1$ on the FWCE (using (7.2)) and this resulted in accurate detection of the fast worms. The profiles that corresponded to the fast worms were filtered off by our window-based adaptive profiler before sending the remaining profiles to the SWCE. This is depicted in Fig. 7.4. On the SWCE, Fig. 7.6 shows that the number of detectors (i.e. SWDAs) that positively detected the slow worms 


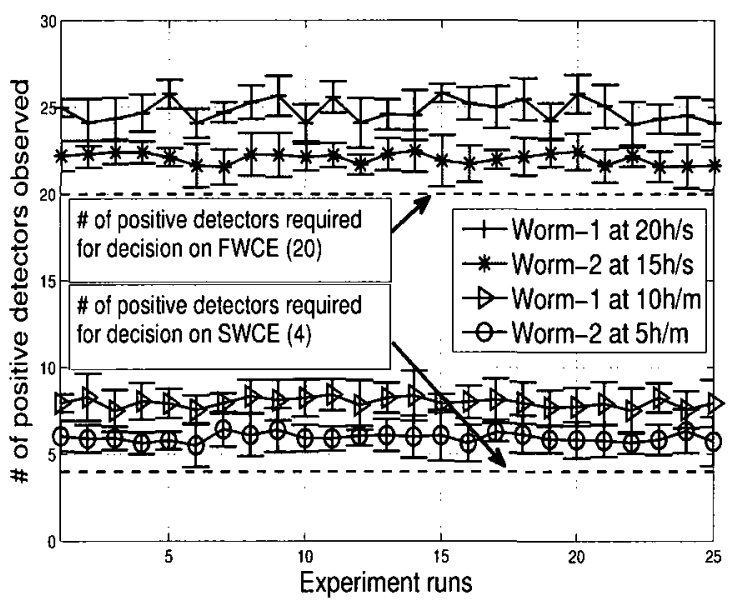

Figure 7.6: Experiment 1: Integrated detection of fast and slow worms. $W=254, \gamma_{f}=2$, $\gamma_{s}=0.1, p_{f}^{s}=p_{f}^{f}=0.01, p_{d}^{s}=p_{d}^{f}=0.99$.

amongst the profiles sent to the SWCE also exceeded the number required for a fused decision $\mathbf{D}^{\mathbf{s}}=1$ on the SWCE (using (7.7)) and this resulted in accurate detection of the slow worms also. The outcome is that both fast and slow worms were detected without the fast worms interfering with slow worm detection. In a typical enterprise network environment with fixed $p_{f}^{s}, p_{f}^{f}, p_{d}^{s}, p_{d}^{f}$ parameters, it is crucial to tune the FWCE and SWCE appropriately with the $\gamma_{f}$ and $\gamma_{s}$ parameters respectively based on observed trends and baseline information gathered from the network.

\subsubsection{Experiment 2: Effect of varying $\gamma_{f}$ and $\gamma_{s}$}

In this experiment, two attacking hosts, one from Network-1 and one from Network-2 in Fig. 7.5 were used to launch different attacks (worm-1 and worm-2 respectively) on hosts in the target networks. The scanning rate of the worm- 1 attack was set to $20 \mathrm{~h} / \mathrm{s}$ to emulate a fast scanning worm. The scanning rate of the worm- 2 attack was set to $10 \mathrm{~h} / \mathrm{m}$ to emulate a slow scanning worm. The objective of the experiment was to demonstrate the effect of varying $\gamma_{f}$ or $\gamma_{s}$ on the integrated detection technique. 
Two sets of experiment runs were made and in each set the value of $\gamma_{f}=\frac{C_{10}^{f}}{C_{01}^{f}}$ or $\gamma_{s}=\frac{C_{10}^{s}}{C_{01}^{s}}$ was modified while keeping the other constant at $\gamma_{s}=0.1$ or $\gamma_{f}=2$ respectively. Parameters $p_{f}^{s}=p_{f}^{f}=0.01, p_{d}^{s}=p_{d}^{f}=0.99$ were used throughout the experiment. Figure 7.7 and Figure 7.8 show the results of the two sets of experiments. In the first set, Figure 7.7 shows that for fast worm detection, an increase in $\gamma_{f}$ (with $\gamma_{s}$ parameter fixed at 0.1 ) resulted in a corresponding increase in the number of positive detectors (i.e. FWDAs) required to meet the conditions for a fused decision $\mathbf{D}^{\mathbf{f}}=1$ on the FWCE using the likelihood ratio test in (7.2). This is similar to the observation made with experiments carried out in section 5.4.5. Note that the $\gamma_{s}$ parameter was fixed at 0.1 to ensure that the number of positive detectors (i.e. SWDAs) required to meet the conditions for a fused decision $\mathbf{D}^{\mathbf{s}}=1$ on the SWCE using (7.7) remained an average of 4.

For the second set of experiments, Figure 7.8 shows that for slow worm detection an increase in $\gamma_{s}$ (with $\gamma_{f}$ parameter fixed at 2) causes an increase in the number of positive detectors (i.e. SWDAs) required by the SWCE to make a fused decision $\mathbf{D}^{\mathbf{s}}=1$. Also, note that the $\gamma_{f}$ parameter was fixed at 2 to ensure that the number of positive detectors (i.e. FWDAs) required to meet the conditions for a fused decision $\mathbf{D}^{\mathbf{f}}=1$ on the FWCE using (7.2) remained an average of 20 .

These results underscore the importance of properly tuning the $\gamma_{s}$ and $\gamma_{f}$ parameters for practical deployments of our GEP-based integrated detection scheme.

\subsubsection{Experiment 3: Effect of varying $p_{f}$ on slow worm detection with background fast scanning benign traffic}

Previous results obtained from experiments performed in Section 5.4.4 show that with parameter $\gamma=2.0$ our EDANC GEP-based algorithm exhibited false detection of benign traffic

for probability of false detection parameter $p_{f} \geq 0.55$ on the host detectors. This experiment 


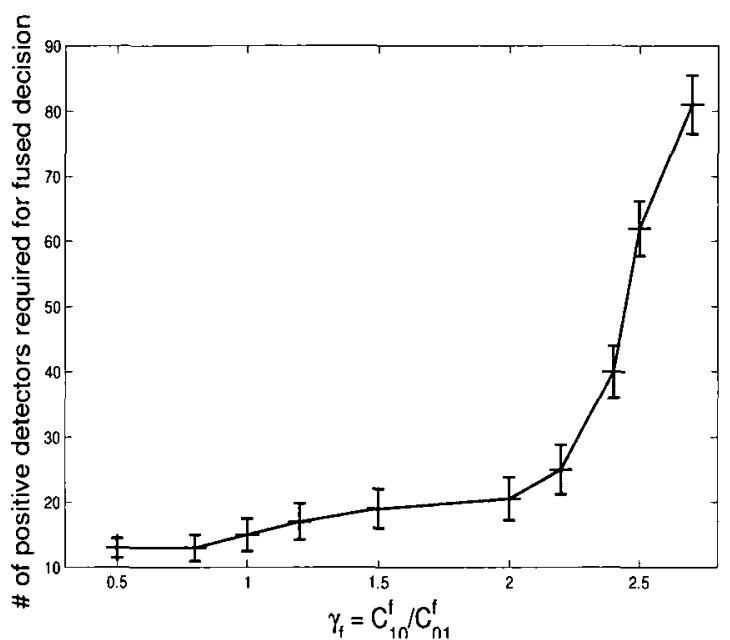

Figure 7.7: Experiment 2: Effect of varying $\gamma_{f}=\frac{C_{10}^{f}}{C_{01}^{f}}$ on fast worm detection in FWCE. $W=254, \gamma_{s}=0.1$. Worm scanning rate $=20 \mathrm{~h} / \mathrm{s}, p_{f}^{s}=p_{f}^{f}=0.01, p_{d}^{s}=p_{d}^{f}=0.99$.

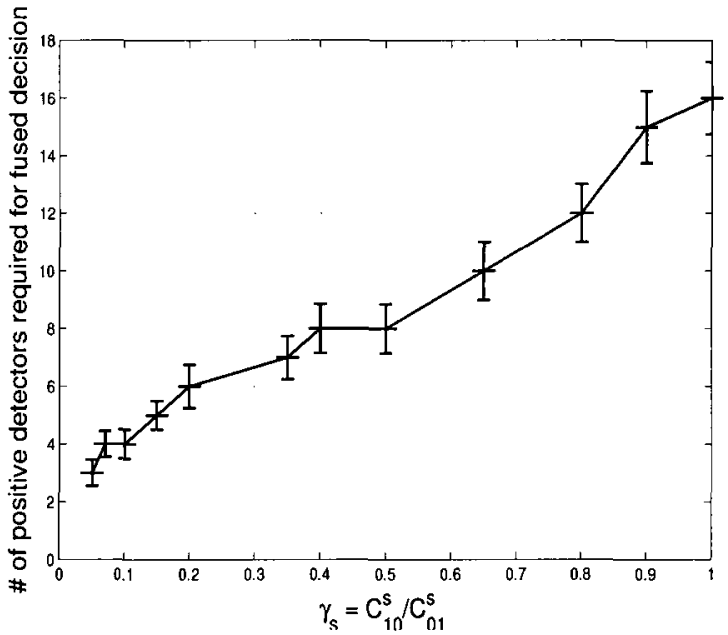

Figure 7.8: Experiment 2: Effect of varying $\gamma_{s}=\frac{C_{10}^{s}}{C_{01}^{s}}$ on slow worm detection in SWCE. $W=254, \gamma_{f}=2$. Worm scanning rate $=10 \mathrm{~h} / \mathrm{m}, p_{f}^{s}=p_{f}^{f}=0.01, p_{d}^{s}=p_{d}^{f}=0.99$.

was designed to investigate the impact of varying $p_{f}^{f}$ on slow worm detection in the presence of fast scanning benign traffic, given that $\gamma_{s}=0.1$ and $\gamma_{f}=2.0$. One host in Network-1 was used to launch direct malicious attacks on hosts in the target networks at a scanning rate of $10 \mathrm{~h} / \mathrm{m}$. A second host in Network-1 was used to scan the target networks with benign traffic at a rate of $15 \mathrm{~h} / \mathrm{s}$. The probability of detection on both FWDA and SWDA was set to 0.99 . 


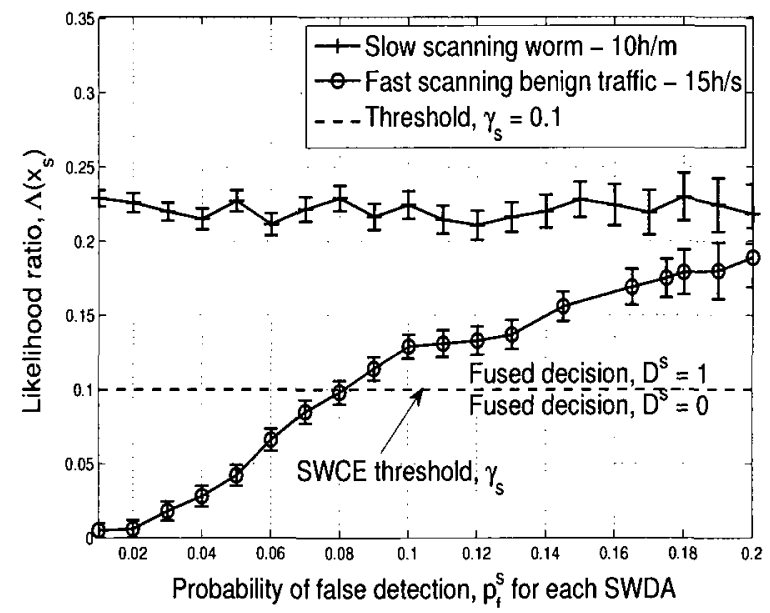

Figure 7.9: Experiment 3: Impact of varying $p_{f}^{s}$ on slow worm detection with background fast scanning benign traffic. $W=254$, Slow worm scanning rate $=10 \mathrm{~h} / \mathrm{m}, \gamma_{s}=0.1, \gamma_{f}=2.0$, $p_{d}^{s}=p_{d}^{f}=0.99, p_{f}^{f}=0.01$.

The probability of false detection on the FWDA, $p_{f}^{f}$ was also set to 0.01 . Several sets of experiment runs were made, and in each set the value of the probability of false detection $p_{f}^{s}$ on the SWDAs in the target network was varied from 0.01 to 0.2. Fig. 7.9 shows that changes in $p_{f}^{s}$ parameter on the SWDA does not have significant impact on the detection of the slow scanning malicious traffic if the $p_{d}^{s}$ parameter on the SWDA is fixed. This is expected given the definition of $p_{f}^{s}$ and $p_{d}^{s}$, expressed in Table 7.4 and is similar to observations made in Section 5.4.4. We observed that the fast scanning benign traffic was not detected as malicious by the FWDA. Therefore, the traffic profile corresponding to the benign traffic was not filtered out on the window-based adaptive profiler (see Fig. 7.4), but was forwarded to the Slow Worm Correlation Engine (SWCE). Fig. 7.9 shows that an increase in $p_{f}^{s}$ on the SWDA results in a corresponding increase in the likelihood that the benign traffic will be falsely detected as malicious on the SWCE. This is because as $p_{f}^{s}$ increases the number SWDAs that wrongly detect the benign traffic as malicious (with $H_{1}$ decisions) increases progressively. We observed in Fig. 7.9 that the benign traffic was falsely detected as malicious only for values of $p_{f}^{s} \geq 0.08$ on the SWDA. In otherwords, for parameter $p_{f}^{s}<0.08$ on the SWDA and $p_{d}^{s}=p_{d}^{f}=0.99, p_{f}^{f}=0.01$, our integrated GEP-based detection tech- 
nique correctly determined that the fast scanning benign traffic was actually benign while accurately detecting the slow scanning malicious traffic. These results and observations can be useful to security engineers in product selection or tuning of host detectors and gateway routers that run our GEP-based integrated detection algorithms.

\subsection{Chapter Summary}

In this chapter, we proposed and implemented modifications to our earlier proposed Endpoint Detection And Network Containment (EDANC) detection technique (presented in Chapter 5) which extend its capabilities to slow worm detection. Using the modified EDANC technique we presented an improved integrated approach to detecting both fast and slow scanning malicious worms even when they occur concurrently in the same computer network. The integrated detection approach is based on the Generalized Evidence Processing (GEP) theory, a decision level multi-sensor data fusion technique. With GEP theory, evidence collected by distributed detectors determine the probability mass associated with a decision under a hypothesis. The probability mass associated with each host detector decision is combined at a fusion center (the gateway router) to arrive at an improved fused detection decision by minimizing a cummulative decision risk function.

We emphasized that slow worms do not necessarily exist alone in computer networks. Typically, malicious intrusion traffic of varying scanning rates occur randomly in computer networks and this can introduce false alarms to detection of slow worms. The integrated detection technique uses a window-based self adapting profiler to filter detected fast scanning malicious traffic profiles hence isolating slow scanning malicious traffic profiles in the correlation engine.

We carried out some experiments with the integrated detection technique on a live test-bed and demonstrated its behavior in detecting both fast and slow scanning malicious worms 
that occur concurrently in the same computer network. Some useful deductions from the experimental results include the following:

1. The integrated detection technique is capable of accurately detecting both fast and slow worms without the fast worms interfering with slow worm detection. However, we acknowledge that our test-bed is not representative of all network scenarios on the Internet. For accurate detection, proper tuning of detection parameters is required.

2. With all other detection parameters kept constant, an increase in $\gamma_{f}=\frac{C_{10}^{f}}{C_{01}^{f}}$ and $\gamma_{s}=$ $\frac{C_{10}^{s}}{C_{01}^{s}}$ results in a corresponding increase in the number of positive detectors (FWDAs and SWDAs) required to meet the conditions for a fused decision $\mathbf{D}^{\mathbf{f}}=1$ on the FWCE and $\mathbf{D}^{\mathbf{s}}=1$ on the SWCE respectively. Conversely, decreasing the $\gamma_{f}$ and $\gamma_{s}$ parameters decreases the number of positive detectors required for a fused decision $\mathbf{D}^{\mathbf{f}}=1$ and $\mathbf{D}^{\mathbf{s}}=1$ on the FWCE and the SWCE respectively.

3. Our GEP-based integrated detection technique is capable of accurately detecting slow scanning malicious traffic even in the presence of background fast scanning benign traffic. It is also capable of correctly determining that the fast scanning benign traffic is actually benign. This improves detection accuracy and minimizes false detection rates. Proper tuning of both host detector and fusion center parameters is required to achieve this. 


\section{Chapter 8}

\section{Conclusion and Future Directions}

\subsection{Summary of Problems}

This thesis primarily investigated the following problems:

1. Detection and containment of fast scanning malicious worms in computer networks with low false detection rates and optimal accuracy irrespective of their signature or their scanning strategies. Detection of such worms with minimal false detection rates and within short intervals still remains a problem today and development of a practical and deployable detection and containment solution remains an open research issue.

2. Detection of both slow scanning stealthy worms and fast scanning malicious worms in computer networks with low false detection rates and optimal accuracy even when they occur concurrently in a target computer network. We point out that there are two major shortfalls of previously proposed approaches to detection of slow scanning and fast scanning worms. First, a common characteristic of previously proposed techniques is the use of connection counts and traffic rates as the basis for anomalous 
detection. This approach inherently carries a high detection rates because worms are capable of propagating at rates similar or less than normal traffic rates and therefore can camouflage as normal traffic [27]. Also, anomalous connection counts and traffic rates (whether high or low) do not necessarily confirm existence of malicious activity. Second, though previously proposed techniques demonstrate capability to detect fast scanning and slow scanning intrusions under certain circumstances, they do not address detection of both fast scanning and slow scanning malicious intrusions when they occur concurrently in the same target computer network.

\subsection{Summary of Contributions}

The first contribution of this thesis to the state of the art is the novel use of the Generalized Evidence Processing (GEP) theory, a decision level multi-sensor data fusion technique for detection of malicious intrusions. Other decision level multi-sensor data fusion techniques, such as the Bayesian theory and the Dempster-Shafer theory have been used in the past as theoretical foundations for intrusion detection, but as far as our literature survey revealed, and to the best of our knowledge, we were the first in [4] to use the GEP theory as a theoretical foundation for intrusion detection of malicious traffic in computer networks. We developed and analysed our novel Endpoint Detection And Network Containment (EDANC) approach for distributed detection and collaborative defense against fast spreading worms. The EDANC detection and correlation engine is based on the GEP theory.

The second contribution of this thesis is the development and analysis of the Analytical Active Worm Containment (AAWC) model. While considering the random scanning behavior of fast spreading malicious worms, we developed the Analytical Active Worm Containment (AAWC) model, a novel discrete-time model which we used to model vulnerable host pop- 
ulation protected from random fast scanning worm attacks as a result of our Endpoint Detection And Network Containment (EDANC) scheme in a large scale network. Using the AAWC model, we demonstrated quantitatively the collaborative containment capability of EDANC defense in a large scale network. The proposed EDANC defense approach uses a network-based collaborative technique for fast containment of fast spreading worms. With the EDANC approach, after the detection and correlation engines identify an offending traffic flow, network filters are automatically generated and deployed on the first upstream router (i.e the gateway router) of the network segment or cell under attack. The gateway router of the target cell then sends notification signals to neighbor routers. Participating neighbor routers that receive the notification signal run a reactive blocking protocol which ensures execution of similar containment and notification actions thus achieving enterprisewide collaborative containment. In Chapter six, the AAWC model was used to quantitatively demonstrate the effectiveness of the EDANC containment technique in protecting a vulnerable uninfected population from infection from a fast scanning malicious worm attack. We define containment of an infectious worm as the complete halting of further worm spread. Therefore, when a fast spreading worm is contained using the EDANC defense technique, significant portions of the vulnerable uninfected population are protected from infection.

The third contribution of this thesis to the state of the art is the proposal and development of a novel approach to integrated detection of both slow scanning and fast scanning malicious worms that is distinct from previously proposed approaches. This involved introducing an improvement to our proposed EDANC detection and correlation engine which extends its capabilities to detection of slow worms. The resulting integrated detection technique was used to achieve detection of both fast scanning and slow scanning malicious worms even when they occur concurrently in a target computer network. Our technique is distinct in a number of ways. First, our proposed detection approach uses an optimized correlation technique based on the GEP theory to combine malicious intrusion evidence and local decisions provided by distributed host-based anomaly intrusion detectors. The use of the GEP 
theory, an optimized multi-sensor data fusion technique for combining the local decisions provided by the individual host detectors ensures that the false detection rates are minimized and that detection accuracy is optimized. The fact that host anomaly detectors are also known to be capable of exhibiting low false detection rates [21] [68] adds to making our novel GEP-based detection technique even more viable. Second, our novel detection approach takes into consideration the real possibility that slow scanning malicious worms can occur concurrently with faster propagating malicious worms in computer networks. This particularly exacerbates the difficulty in detecting slow worms due to interference from other traffic flows scanning at faster rates.

\subsection{Summary of Thesis Content}

The chronology of the content of this thesis are as follows:

Chapter one presented the objectives and motivations for this thesis work including a general description of detection and defense techniques against active malicious worms. Chapter two presented the thesis approach and contributions made by this thesis to the state of the art in the area of detection and containment of active malicious worms. In Chapter three and Chapter four, we reviewed previous related work and discussed relevant background for decision level multi-sensor data fusion which formed the theoretical foundation for our proposed detection approach.

In Chapter five, our novel Endpoint Detection And Network Containment (EDANC) approach for distributed detection and collaborative defense against fast spreading worms was introduced and analysed. The EDANC detection and correlation engine is based on the GEP theory, a decision level multi-sensor data fusion technique. With GEP theory, the evidence collected by the distributed detectors determines the probability associated with a decision under a hypothesis. The probability mass associated with each host detector decision is 
combined at a fusion center (the gateway router) to arrive at an optimal fused detection decision by minimizing a cummulative decision risk function. The GEP theory is known to have advantages over the two major evidence combining theories that have dominated the field of distributed evidence processing - the Bayesian theory and the Dempster-Shafer theory.

We presented an analysis of EDANC detection interval for a fast scanning worm and experimented with the EDANC scheme on a live test-bed. Results obtained from the experiments concurred with analytical results. The results also validate the functionality of the EDANC detection technique for fast scanning malicious worms. Some useful deductions from the experimental results include the following:

1. The EDANC scheme is capable of automatically detecting malicious intrusion attacks with scanning rate of over $15 \mathrm{~h} / \mathrm{s}$ within an average of 8 seconds after starting the attack on a network with cells (network segments) containing 254 hosts.

2. A reduction in the probability of detection, $p_{d}$ of the individual detectors results in a corresponding increase in the number of positive detectors required to meet the conditions for a fused decision $\mathbf{D}=1$ (i.e. decision that observed intrusion is malicious). We used homogeneous host detectors with same $p_{d}$ and $p_{f}$ values. Though somewhat intuitive, the results showed that with poor detectors (with low $p_{d}$ values) the correlation algorithm required more reports of individual detector local decisions to arrive at a fused decision. Conversely, more accurate detectors (with higher $p_{\boldsymbol{d}}$ values) caused the correlation algorithm to require less number of individual detector local decisions to arrive at a fused decision.

3. Changes in probability of false detection, $p_{f}$ values for individual detectors does not have significant impact on the detection of malicious traffic if the $p_{d}$ value for the detectors is fixed. This is expected since detection of malicious traffic is dependent on $p_{d}$ but has no dependency on $p_{f}$ (see Table 5.1). However, an increase in $p_{f}$ results in 
a corresponding increase in the likelihood that a benign traffic will be falsely detected as malicious. With $p_{f} \geq 0.55$ our GEP algorithm exhibited false detection of benign traffic.

4. An increase in $\gamma=\frac{C_{10}}{C_{01}}$ (i.e. ratio of the cost of a false positive decision to the cost of a false negative decision) results in a corresponding increase in the number of positive detectors required to meet the conditions for a fused decision $\mathbf{D}=1$.

5. Generated ROC curves show that the EDANC GEP-based detection technique exhibits much better accuracy than the use of a single host detector for detection.

6. In multiple concurrent malicious attack scenarios, even though all the malicious attack profiles are identified as malicious and selected for containment, the profiles are blocked (or contained) in a sequence ordered by the combined probability of detection $\left(P_{D_{i}}\right)$ computed for the profiles. In our experiments, we observed that the scanning rate of a worm had a direct relationship with $P_{D_{i}}$ computed for that worm profile. The worm with a faster scanning rate exhibited a greater $P_{D_{i}}$ and was blocked first.

In Chapter six, considering the random scanning behavior of malicious worms, we developed the Analytical Active Worm Containment (AAWC) model, a discrete-time model used to model vulnerable host population protected as a result of the EDANC scheme in a large scale network. Using the AAWC model, we demonstrated quantitatively the collaborative containment capability of EDANC. For a scanning worm attack, the AAWC model assumes that successful direct worm scans are stopped when the number of protected hosts (modeled using our AAWC model) exceed the number of directly scanned hosts (modeled using the known AAWP model [19]), thus preventing further increase in the number of hosts infected by direct scans. Our results showed that this is achievable within 13 seconds after release of the worm. In addition, it was observed that while network containment of worms can halt further worm spread due to direct scans, it does not recover infected hosts nor does it prevent local scanning within a protected cell (or network segment). Immunization by patching was 
therefore introduced to EDANC by introducing a non-zero patching rate to the AAWP model. This combined approach (EDANC network containment and immunization) caused the number of infected hosts after EDANC defense to decrease by the patching rate on every subsequent time tick. Results showed that the number of infected hosts in protected cells was significantly reduced and tended towards zero as a result of this combined approach, thus ensuring that hosts infected before EDANC defense did not become launching platforms for more attacks within or outside their network cells.

In Chapter seven, we implemented improvements to our earlier proposed Endpoint Detection And Network Containment (EDANC) detection technique which extended its capabilities to slow worm detection. Using the improved EDANC technique we presented an integrated approach to detecting both fast and slow scanning malicious worms even when they occur concurrently in the same computer network. We emphasized that slow worms do not necessarily exist alone in computer networks. Typically, malicious intrusion traffic of different scanning rates (fast and slow) can co-exist on the Internet and in computer networks, and their random co-existence can introduce false alarms to detection of slow worms in particular. The integrated detection technique uses a window-based self adapting profiler to filter detected fast scanning malicious traffic profiles hence isolating detected slow scanning malicious traffic profiles in the correlation engine. We carried out experiments with the integrated detection technique on a live test-bed and demonstrated its behavior in detecting both fast and slow scanning malicious worms that occur concurrently. Some useful deductions from the experimental results include the following:

1. The integrated detection technique is capable of accurately detecting both fast and slow worms without the fast worms interfering with slow worm detection. However, we acknowledge that our test-bed is not representative of all network scenarios on the Internet. For accurate detection, proper tuning of detection parameters is required.

2. With all other detection parameters kept constant, an increase in $\gamma_{f}=\frac{C_{10}^{f}}{C_{01}^{f}}$ and $\gamma_{s}=$ 
$\frac{C_{10}^{s}}{C_{01}^{s}} *$ results in a corresponding increase in the number of positive detectors (FWDAs and SWDAs) required to meet the conditions for a fused decision $\mathbf{D}^{\mathbf{f}}=1$ on the FWCE and $\mathbf{D}^{\mathbf{s}}=1$ on the SWCE respectively. Conversely, decreasing the $\gamma_{f}$ and $\gamma_{s}$ parameters decreases the number of positive detectors required for a fused decision $\mathbf{D}^{\mathbf{f}}=1$ and $\mathbf{D}^{\mathbf{s}}=1$ on the FWCE and the SWCE respectively.

3. Our GEP-based integrated detection technique is capable of accurately detecting slow scanning malicious traffic even in the presence of background fast scanning benign traffic. It is also capable of correctly determining that the fast scanning benign traffic is actually benign. This improves detection accuracy and minimizes false detection rates. Proper tuning of both host detector and fusion center parameters is required to achieve this.

\subsection{Future Directions}

For future work, a number of directions are possible:

1. One interesting direction would be to extend the work on integrated host-based anomaly detection of worms to overlay networks such as peer-to-peer networks and ad hoc networks. Since the EDANC detection scheme relies on host detectors for local detection, and gateway routers for correlation and fused decision, it is possible to create virtual networks that run the EDANC protocols and overlay such virtual networks on existing traditional IP networks. Also, several routers that form part of the virtual network can participate in the EDANC collaborative containment protocol.

2. In this thesis, we developed a detection scheme for slow scanning malicious worms

\footnotetext{
${ }^{*} \gamma_{f}$ is the ratio of the cost of a false positive decision to the cost of a false negative decision on the FWCE, while $\gamma_{s}$ is the ratio of the cost of a false positive decision to the cost of a false negative decision on the SWCE.
} 
even in the presence of faster scanning malicious traffic. With proliferation of Web 2.0 and peer-to-peer social networks, a new vulnerability and threat model due to stealth worms which are slowly propagating but with no peculiar communication patterns [67], seem quite conceivable. Successful large scale infection as a result of such worms can be exploited by bot herds and used to perpetuate significant damage on computer systems and networks. More work is required to develop adequate defenses against this special class of worms. We believe that multi-sensor data fusion such as the GEP theory holds promise in this area.

3. Another possible direction involves investigating the impact of indecisive detectors on GEP theory-based intrusion detection of malicious worms. In this thesis, our implementation of the GEP theory focussed on the use of local detectors that are capable of making a decision on the malicious or benign nature of observed traffic profiles. We did not investigate any scenarios with indecisive detectors (such as poor quality detectors) that are not capable of making such a decision. More work is required to understand the implication of using the GEP theory as a theoretical foundation for intrusion detection of malicious worms in computer networks when hard local decisions about local detector observations cannot be made. 


\section{References}

[1] J.M. Agosta, C. Diuk-Wasser, J. Chandrashekar, and C. Livadas. An adaptive anomaly detector for worm detection. In SYSML'07: Proceedings of the 2nd USENIX workshop on Tackling computer systems problems with machine learning techniques, pages 1-6, Berkeley, CA, USA, 2007. USENIX Association.

[2] F. Akujobi, I. Lambadaris, and E. Kranakis. Endpoint-driven intrusion detection and containment of fast spreading worms in enterprise networks. In IEEE Military Communications Conference (MILCOM) 2007, pages 1-7, Orlando, FL, USA, 2007. IEEE.

[3] F. Akujobi, I. Lambadaris, and E. Kranakis. Modeling host-based detection and active worm containment. In CNS '08: Proceedings of the 11th communications and networking simulation symposium, pages 222-229, New York, NY, USA, 2008. ACM.

[4] F. Akujobi, I. Lambadaris, and E. Kranakis. Detection of Slow Malicious Worms using Multi-sensor Data Fusion. IEEE Symposium on Computational Intelligence for Security and Defense Applications (CISDA 2009), 2009.

[5] F. Akujobi, I. Lambadaris, and E. Kranakis. An Integrated Approach to Detection of Fast and Slow Scanning Worms. In ASIACCS '09: Proceedings of the 4th International Symposium on Information, Computer, and Communications Security, pages 80-91, New York, NY, USA, 2009. ACM.

[6] F. Akujobi, I. Lambadaris, and E. Kranakis. Towards Host-based Detection and Col- 
laborative Network Containment of Fast Spreading Active Worms. Journal of Network and Computer Applications, 2009 - submitted and awaiting review.

[7] D. Alderson, L. Li, W. Willinger, and J.C. Doyle. Understanding Internet topology: Principles, models, and validation. In IEEE/ACM Transactions on Networking, 13(6):1205-1218, 2005.

[8] K.G. Anagnostakis, M.B. Greenwald, S. Ioannidis, A.D. Keromytis, and D. Li. A cooperative immunization system for an untrusting Internet. In 11th IEEE International Conference on Networking (ICON), pages 403-408, 2003.

[9] F. Apap, A. Honig, S. Hershkop, E. Eskin, and S.J. Stolfo. Detecting malicious software by monitoring anomalous windows registry accesses. In In Proceedings of the Fifth International Symposium on Recent Advances in Intrusion Detection (RAID-2002, pages 16-18, Zurich, Switzerland, 2002.

[10] P. Barford, J. Kline, D. Plonka, and R. Amos. A signal analysis of network traffic anomalies. In IMW '02: Proceedings of the 2nd ACM SIGCOMM Workshop on Internet measurment, pages 71-82, New York, NY, USA, 2002. ACM.

[11] T. Bass. Multisensor data fusion for next generation distributed intrusion detection systems. In Proceedings of the IRIS National Symposium on Sensor and Data Fusion, pages 24-27, 1999.

[12] T. Bass. Intrusion detection systems and multisensor data fusion. Commun. ACM, $43(4): 99-105,2000$.

[13] T. Bayes. An essay towards solving a problem in the doctrine of chances. Philosophical Transactions of the Royal Society, 53:370-418, 1763.

[14] D. Brumley, L. Liu, P. Poosankam, and D. Song. Design space and analysis of worm defense strategies. In ASIACCS '06: Proceedings of the 2006 ACM Symposium on Information, computer and communications security, pages 125-137, New York, NY, USA, 2006. ACM. 
[15] M. Burgess. Probabilistic anomaly detection in distributed computer networks. Science of Computer Programming, 60(1):1-26, March 2006.

[16] M. Cai, K. Hwang, Y. Kwok, S. Song, and Y. Chen. Collaborative Internet worm containment. IEEE Security and Privacy, 3(3):25-33, 2005.

[17] S.G. Cheetancheri, J.M. Agosta, D.H. Dash, K.N. Levitt, J. Rowe, and E.M. Schooler. A distributed host-based worm detection system. In LSAD '06: Proceedings of the 2006 SIGCOMM workshop on Large-scale attack defense, pages 107-113, New York, NY, USA, 2006. ACM.

[18] Q. Chen and U. Aickelin. Dempster-Shafer for anomaly detection. CoRR, abs/0803.1568, 2008.

[19] Z. Chen, L. Gao, and K. Kwiat. Modeling the spread of active worms. In INFOCOM 2003, 22nd Annual Joint Conference of the IEEE Computer and Communications., pages $1890-1900,2003$.

[20] Computer Economics Inc. (2007). Malware report: The economic impact of viruses, spyware, adware, botnets, and other malicious code. Computer Economics Inc., Irvine, CA - http://www.computereconomics.com/page.cfm?name=Malware\%20Report, 2007. Website was available in November 2009.

[21] M. Costa, J. Crowcroft, M. Castro, A. Rowstron, L. Zhou, L. Zhang, and P. Barham. Vigilante: End-to-end containment of Internet worms. In Proceedings of the Symposium on Systems and Operating Systems Principles (SOSP), pages 133-147, 2005.

[22] D. Dash, B. Kveton, J.M. Agosta, E. Schooler, J. Chandrashekar, A. Bachrach, and A. Newman. When gossip is good: distributed probabilistic inference for detection of slow network intrusions. In $A A A I^{\prime} 06$ : proceedings of the 21st national conference on Artificial intelligence, pages 1115-1122. AAAI Press, 2006.

[23] eEye Digital Security. Coderedii worm analysis. eEye Digital Security article - 
http://research.eeye.com/html/advisories/published/AL20010804.html, 2001. Website was available in November 2009.

[24] eEye Digital Security. Blaster worm analysis. eEye Digital Security article http://research.eeye.com/html/advisories/published/AL20030811.html, 2003. Website was available in November 2009.

[25] P. Fogla and W. Lee. Evading network anomaly detection systems: formal reasoning and practical techniques. In $C C S$ '06: Proceedings of the 13th ACM conference on Computer and communications security, pages 59-68, New York, NY, USA, 2006. ACM.

[26] J.C. Frauenthal. Mathematical Modeling in Epidemiology. New York: Springer-Verlag, 1980.

[27] C. Gates and C. Taylor. Challenging the anomaly detection paradigm: A provocative discussion. In NSPW'06: Proceedings of the 2006 workshop on New security paradigms, pages 21-29, New York, NY, USA, 2006. ACM.

[28] G. Gu, A. A. Cárdenas, and W. Lee. Principled reasoning and practical applications of alert fusion in intrusion detection systems. In ASIACCS '08: Proceedings of the 2008 ACM symposium on Information, computer and communications security, pages 136-147, New York, NY, USA, 2008. ACM.

[29] A. Habib, M. Hefeeda, and B. Bhargava. Detecting service violations and DoS attacks. In In.Proceedings of 2003 Internet Society Symposium on Network and Distributed System Security (NDSS) 2003, pages 177-189, 2003.

[30] D. L. Hall and J. Llinas. An introduction to multisensor data fusion. Proceedings of the IEEE, 85(1):6-23, 1997.

[31] D.L. Hall and S.A.H. McMullen. Mathematical Techniques in Multisensor Data Fusion. Artech House, Inc., Norwood, MA, USA, 2004. 
[32] M. Handley, C. Kreibich, and V. Paxson. Network intrusion detection: evasion, traffic normalization, and end-to-end protocol semantics. In SSYM'01: Proceedings of the 10th conference on USENIX Security Symposium, page 9, Berkeley, CA, USA, 2001. USENIX Association.

[33] N. Hanebutte and P.W. Oman. Software vulnerability mitigation as a proper subset of software maintenance. Journal of Software Maintenance, 17(6):379-400, 2005.

[34] J. Ioannidis and S.M. Bellovin. Implementing pushback: Router-based defense against DDoS attacks. In Proceedings of Network and Distributed System Security Symposium (NDSS), pages 100-108, Reston, VA, USA, 2002.

[35] J. Jung, R.A. Milito, and V. Paxson. On the Adaptive Real-Time Detection of FastPropagating Network Worms. Springer Berlin/Heidelberg, 2007.

[36] J. Jung, V. Paxson, A. Berger, and H. Balakrishnan. Fast Portscan Detection Using Sequential Hypothesis Testing. IEEE Symposium on Security and Privacy, pages 211$225,2004$.

[37] G. Kataria, G. Anand, R. Araujo, R. Krishnan, and A. Perrig. A distributed stealthy coordination mechanism for worm synchronization. In Proceedings of IEEE Securecomm and Workshops, 2006, pages 1-8, 2006.

[38] J.O. Kephart and S.R. White. Measuring and modeling computer virus prevalence. In SP '93: Proceedings of the 1993 IEEE Symposium on Security and Privacy, page 2, Washington, DC, USA, 1993. IEEE Computer Society.

[39] L.A. Klein. Sensor and Data Fusion: A Tool for Information Assessment and Decision Making (SPIE Press Monograph Vol. PM138). SPIE - International Society for Optical Engineering, 2004.

[40] R. Lemos. Attack knocks out microsoft web sites. CNET News.com http://news.cnet.com/2100-1001-251573.html, 2001. Website was available in November 2009 . 
[41] R. Lemos. Hackers cripple white house site. CNET News.com http://news.cnet.com/2100-1001-257068.html, 2001. Website was available in November 2009 .

[42] L. Li, D. Alderson, W. W. Willinger, and J. Doyle. A first-principles approach to understanding the Internet's router-level topology. In SIGCOMM '04: Proceedings of the 2004 conference on Applications, technologies, architectures, and protocols for computer communications, pages 3-14, New York, NY, USA, 2004. ACM.

[43] Q. Liu and H. Wang. A case study on multisensor data fusion for imbalance diagnosis of rotating machinery. Artif. Intell. Eng. Des. Anal. Manuf., 15(3):203-210, 2001.

[44] M. Locasto, K. Wang, A. Keromytis, and S. Stolfo. FLIPS: hybrid adaptive intrusion prevention. In 8th International Symposium on Recent Advanced in Intrusion Detection (RAID), pages 82-101, 2005.

[45] M. Mahoney and P. Chan. An analysis of the 1999 DARPA/Lincoln laboratory evaluation data for network anomaly detection. In In Proceedings of the Sixth International Symposium on Recent Advances in Intrusion Detection, pages 220-237. Springer-Verlag, 2003.

[46] J. Markoff. Worm infects millions of computers worldwide. The New York Times - http://www.nytimes.com/2009/01/23/technology/internet/23worm.html, 2009. Website was available in November 2009.

[47] Thomas M.C. and V. Venkataramanan. Dempster-Shafer theory for intrusion detection in ad hoc networks. IEEE Internet Computing, 9(6):35-41, 2005.

[48] D. Moore, V. Paxson, S. Savage, C. Shannon, S. Staniford, and N. Weaver. Inside the slammer worm. IEEE Security and Privacy, 1(4):33-39, 2003.

[49] D. Moore, C. Shannon, and K. Claffy. Code Red: A case study on the spread and victims of an Internet worm. In ACM SIGCOMM Internet Measurement Workshop, pages $273-284,2002$. 
[50] D. Moore, C. Shannon, G. Voelker, and S. Savage. Internet quarantine: Requirements for containing self-propagating code. In INFOCOM 2003, 22nd Annual Joint Conference of the IEEE Computer and Communications, pages 1901-1910, 2003.

[51] D. Mutz, F. Valeur, C. Kruegel, and G. Vigna. Anomalous system call detection. ACM Transactions on Information and System Security, 9:61-93, 2006.

[52] Network Security Resources. Governmentsecurity.org. http://www. governmentsecurity.org/forum/index.php?showtopic=4726, 2003. Website was available in November 2009.

[53] D. Nicol and M. Liljenstam. Models and analysis of active worm defense. In $M M M$ ACNS, pages 38-53, 2005.

[54] D. M. Nicol, S. Hanna, S. Frank, and W. H. Sanders. Modeling and analysis of worm defense using stochastic activity networks. In SpringSim '07: Proceedings of the 2007 spring simulation multiconference, pages 349-355, San Diego, CA, USA, 2007. Society for Computer Simulation International.

[55] D.M. Nicol. The impact of stochastic variance on worm propagation and detection. In WORM '06: Proceedings of the 4th ACM workshop on Recurring malcode, pages 57-64, New York, NY, USA, 2006. ACM.

[56] D. Nojiri, J. Rowe, and K. Levitt. Cooperative response strategies for large scale attack mitigation. In 3rd DARPA Information Survivability Conference and Exposition, DISCEX (1), pages 293-302, 2003.

[57] B. Prince. Storm worm continues to spread around globe. FOXNews.com http://www.foxnews.com/story/0,2933,247098,00.html, 2007. Website was available in November 2009.

[58] P. Robert. Zotob, pnp worms slam 13 daimlerchrysler plants. eWEEK.com, Security - http://www.eweek.com/c/a/Security/Zotob-PnP-Worms-Slam-13-DaimlerChryslerPlants/, 2005. Website was available in November 2009. 
[59] K. R. Rohloff and T. Bacşar. Deterministic and stochastic models for the detection of random constant scanning worms. ACM Trans. Model. Comput. Simul., 18(2):1-24, 2008.

[60] S.E Schechter, J. Jung, and A.W. Berger. Fast Detection of Scanning Worm Infections. In 7th International Symposium on Recent Advances in Intrusion Detection (RAID), pages 59-81, French Riviera, France, September 2004.

[61] V. Sekar, Y. Xie, M.K. Reiter, and H. Zhang. A multi-resolution approach for worm detection and containment. In DSN '06: Proceedings of the International Conference on Dependable Systems and Networks, pages 189-198, Washington, DC, USA, 2006. IEEE Computer Society.

[62] C. Shannon and D. Moore. The spread of the witty worm. IEEE Security and Privacy, 2(4):46-50, 2004.

[63] C. Siaterlis and B. Maglaris. Towards multisensor data fusion for DoS detection. In Proceedings of the 2004 ACM symposium on Applied computing, pages 439-446. ACM Press, 2004.

[64] S. Singh, C. Estan, G. Varghese, and S. Savage. Automated worm fingerprinting. In Operating Systems Design and Implementation (OSDI), Proceedings of the 6th conference on Symposium on Operating Systems Design Implementation - Volume 6, pages $45-60,2004$.

[65] S. Stafford, J. Li, and T. Ehrenkranz. Enhancing SWORD to detect zero-day-worminfected hosts. Simulation, 83(2):199-212, 2007.

[66] S. Staniford, D. Moore, V. Paxson, and N. Weaver. The top speed of flash worms. In WORM '04: Proceedings of the 2004 ACM workshop on Rapid malcode, pages 33 42, New York, NY, USA, 2004. ACM.

[67] S. Staniford, V. Paxson, and N. Weaver. How to own the Internet in your spare time. 
In Proceedings of the 11th USENIX Security Symposium, pages 149-167, Berkeley, CA, USA, 2002. USENIX Association.

[68] C. Sullivan, J. Asher, and P. Mauvais. Advanced Host Intrusion Prevention with CSA. Cisco Press, 2006.

[69] Swsoft. Openvz homepage. http://wiki.openvz.org/Main_Page, 2008. Website was available in November 2009.

[70] Cisco Systems Inc. Cisco Catalyst 6500 Supervisor Engine 32 PISA. http:// www.cisco.com/en/US/products/ps7209/index.html, 2008. Website was functional in November 2009.

[71] A. Takanen, P. Vuorijärvi, M. Laakso, and J. Röning. Agents of responsibility in software vulnerability processes. Ethics and Inf. Technol., 6(2):93-110, 2004.

[72] S.C.A. Thomopoulos. Sensor integration and data fusion. Journal of Robotic Systems, $7(3): 337-372,1990$.

[73] S.C.A. Thomopoulos. Theories in distributed data fusion: Comparison and generalization. SPIE, 1383:623-634, 1990.

[74] H.J. Wang, C. Guo, D. Simon, and A. Zugenmaier. Shield: Vulnerability-driven network filters for preventing known vulnerability exploits. In $A C M$ SIGCOMM, pages 193-204, 2004.

[75] J. Wang and Y. Gao. Multi-sensor data fusion for land vehicle attitude estimation using a fuzzy expert system. Data Science Journal, 4:127-139, 2005.

[76] K. Wang and S.J. Stolfo. Anomalous payload-based network intrusion detection. In 7th International Symposium on Recent Advanced in Intrusion Detection (RAID), pages $203-222,2004$.

[77] N. Weaver, S. Staniford, and V. Paxson. Very fast containment of scanning worms. In 
SSYM'04: Proceedings of the 13th conference on USENIX Security Symposium, page 3, Berkeley, CA, USA, 2004. USENIX Association.

[78] G. White and D. DiCenso. Information sharing needs for national security. In HICSS '05: Proceedings of the Proceedings of the 38th Annual Hawaii International Conference on System Sciences (HICSS'05) - Track 5, page 125.3, Washington, DC, USA, 2005. IEEE Computer Society.

[79] D. Whyte, E. Kranakis, and P. Van Oorschot. DNS-based detection of scanning worms in an enterprise network. In Network and Distributed Systems Symposium (NDSS), pages 181-195, 2005.

[80] D. Whyte, P.C. Van Oorschot, and E. Kranakis. Detecting intra-enterprise scanning worms based on address resolution. In ACSAC '05: Proceedings of the 21st Annual Computer Security Applications Conference, pages 371-380, Washington, DC, USA, 2005. IEEE Computer Society.

[81] C. Wong, C. Wang, D. Song, S. Bielski, and G. R. Ganger. Dynamic quarantine of internet worms. In DSN '04: Proceedings of the 2004 International Conference on Dependable Systems and Networks, page 73, Washington, DC, USA, 2004. IEEE Computer Society.

[82] H. Wu, M. Siegel, R. Stiefelhagen, and J. Yang. Sensor fusion using dempster-Shafer theory. In Proceedings of IEEE Instrumentation and Measurement Technology Conference, pages 21-23, 2002.

[83] T. Young. Cyber war moves up NATO agenda. Computing http://www.computing.co.uk/computing/analysis/2202524/cyber-war-moves-natoagenda-3602322, 2007. Website was available in November 2009.

[84] C. C. Zou, L. Gao, W. Gong, and D. Towsley. Monitoring and early warning for Internet worms. In CCS '03: Proceedings of the 10th ACM conference on Computer and communications security, pages 190-199, New York, NY, USA, 2003. ACM. 
[85] C.C. Zou, W. Gong, and D. Towsley. Code Red worm propagation modeling and analysis. In CCS '02: Proceedings of the 9th ACM conference on Computer and communications security, pages 138-147, New York, NY, USA, 2002. ACM.

[86] C.C. Zou, D. Towsley, and W. Gong. Email worm modeling and defense. In 13th International Conference on Computer Communications and Networks, pages 409-414, 2004. 